\title{
Rub-Impact Force Induces Periodic, Quasiperiodic, and Chaotic Motions of a Controlled Asymmetric Rotor System
}

\author{
N. A. Saeed $\left(\mathbb{D},{ }^{1}\right.$ Emad Mahrous Awwad $\left(D,{ }^{2}\right.$ Ali Maarouf $\mathbb{D}^{2},{ }^{2}$ Hassan M. H. Farh $\left(\mathbb{D},{ }^{2}\right.$ \\ Fahd A. Alturki $\mathbb{D}^{2},{ }^{2}$ and Jan Awrejcewicz $\mathbb{D}^{3}$ \\ ${ }^{1}$ Department of Physics and Engineering Mathematics, Faculty of Electronic Engineering, Menoufia University, \\ Menouf 32952, Egypt \\ ${ }^{2}$ Electrical Engineering Department, College of Engineering, King Saud University, P.O. Box 800, Riyadh 11421, Saudi Arabia \\ ${ }^{3}$ Department of Automation, Biomechanics, and Mechatronics, Faculty of Mechanical Engineering, Lodz 90924, \\ Lodz University of Technology, Poland \\ Correspondence should be addressed to N. A. Saeed; nasser.a.saeed@el-eng.menofia.edu.eg
}

Received 11 May 2021; Revised 16 July 2021; Accepted 28 July 2021; Published 12 August 2021

Academic Editor: Gerardo Silva-Navarro

Copyright (c) 2021 N. A. Saeed et al. This is an open access article distributed under the Creative Commons Attribution License, which permits unrestricted use, distribution, and reproduction in any medium, provided the original work is properly cited.

\begin{abstract}
This article aims to explore the oscillatory characteristics of a controlled asymmetric rotor system when subjected to rub and impact forces between the rotor and stator. Four electromagnetic poles are used to control the whirling motion of the rotor system through a linear proportional-derivative control law. The equations of motion that govern the whole system dynamics are derived including the rub and impact forces. The derived mathematical model is analyzed in two basic steps. Firstly, the obtained model is treated as a weakly nonlinear system using perturbation analysis to obtain the slow-flow modulating equations when neglecting the rub and impact forces. Depending on the obtained slow-flow equations, different response curves are plotted to explore the system's periodic vibrations and determine the conditions at which the system can exhibit rub and impact force. Secondly, the whole system model including the rub and impact forces is investigated by using the bifurcation diagrams, Poincare map, frequency spectrums, and temporal oscillations. The obtained results revealed that the applied control law could mitigate the system whirling oscillations and prevent the rub and impact forces if the control gains are tuned properly. However, the system can perform period-n, quasiperiodic, or chaotic motion depending on the shaft spinning speed if the controller fails to eliminate the contact between the rotor and stator.
\end{abstract}

\section{Introduction}

The rotating machinery has many engineering applications and is considered as the backbone of a huge number of industries. Therefore, ensuring safe operation of such machines without any catastrophic failure under different operational conditions is the vital role of the scientists and engineers. One of the main reasons that causes failure of rotating machines is nonlinear vibrations. There are many sources for the rotating shaft oscillations such as the shaft's eccentricity, the improper coupling in the case of multirotor systems, the development of the shaft cracks due to the working conditions, the existence of wears, the occurrences of the rub-impact force between the rotating parts and housing, the shaft's asymmetry, and so on. Accordingly, vibration analysis and control of the rotors are the main research subjects of many researchers for a long time. Iwatsubo et al. [1] investigated free and forced vibrations of an asymmetric rotor when supported by asymmetric bearings. The authors applied the asymptotic analysis to find an approximate solution for the system mathematical model, where the obtained results showed that the asymmetry of shaft stiffness coefficients and/or the oil whip of the oil film bearings can destabilize the system motion. Park [2] and Hsieh et al. [3] explored the oscillatory behaviours of the two-pole rotor system. They reported that the system may oscillate with high vibration amplitudes when the shaft angular speed is close to twice the shaft critical speed. Ishida 
and Liu [4] utilized a discontinuous-spring characteristic to stabilize the unstable motion of an asymmetric shaft system, asymmetric rotor system, and rotor system partially filled with liquid. Shahgholi and Khadem [5] investigated the nonlinear vibrations of an asymmetric rotating shaft system in both the primary and parametric resonance cases. The authors considered that both the moment of inertial and bending stiffness are the main sources of the shaft asymmetry. Han and Chu $[6,7]$ and Saeed et al. [8-10] studied the dynamical behaviours of an asymmetric rotor system with a transverse crack. The main conclusion is that the increase of the crack size can stabilize the system's unstable motion. Xiang et al. [11] studied the parametric instability of an asymmetric rotor system having a transverse crack when subjected to both rub-impact and oil-film forces. They investigated the system model using the frequency spectrum, Poincare map, and bifurcation diagram. It is concluded that the crack depth and the stator stiffness have an important role in both the system instability and oscillation amplitudes. Przybylowicz et al. [12] studied the influence of the kinematic excitation on the asymmetric rotor numerically. They showed that the proper design of both the system stiffness coefficient and damping parameter can eliminate the system's chaotic motion. Saeed [13] investigated the nonlinear dynamics of an asymmetric rotor supported vertically. The obtained results showed that the asymmetry of the shaft stiffness coefficients can induce a backward whirling motion. In addition, the authors reported that the asymmetric system may have four stable periodic solutions at the same angular speed. Saeed [14] explored the dynamical behaviours of an asymmetric horizontally suspended rotor system. The author obtained an analytical solution for the studied system applying perturbation analysis. He showed that the asymmetry of the linear stiffness coefficient can suppress the system's backward whirling motion. Felix et al. [15] performed numerical simulations for the oscillatory behaviours of both the one and two-disk rotor system when excited by a nonideal motor.

On the other hand, many researchers introduced different passive and active control techniques to suppress or mitigate the undesired vibrations of the rotating machines. For example, Tehrani et al. [16, 17] employed both the nonlinear energy sinks and vibration absorber as passive control methods to mitigate the vibrations of the rotating machine. Ishida and Inoue [18] applied a nonlinear active vibration absorber to control the lateral vibrations of a vertically suspended rotating shaft. Saeed et al. [19-22] applied different active control strategies utilizing the active magnetic bearings as an actuator. Saeed and Kamel [19] employed a nonlinear combination of both the position and velocity controllers to control the vibrations of the nonlinear Jeffcott rotor model supported horizontally via four electromagnetic poles. Saeed et al. [20] proposed a combination of both the nonlinear and linear proportional-derivative controllers to mitigate the nonlinear vibrations of the asymmetric rotor system that is supported vertically. Saeed et al. [21, 22] introduced six electromagnetic poles to control the rotating disk vibrations utilizing PD controller. They explored two control configurations that are the Cartesian and radial control schemes. It is reported that the Cartesian control scheme has high efficiency than the radial control technique in mitigating the system vibrations. However, they concluded that the radial control strategy has high robustness against the system instability. Piccirillo et al. [23] investigated the impact force elimination and chaos control utilizing magnetorheological fluid. The authors applied the damped passive control technique to suppress the system's chaotic oscillations, while a fuzzy logic controller is proposed to control the system's lateral vibrations. Singh et al. [24] explored the unique nonlinear features of the permanent magnet synchronous motor (PMSM) system. The authors reported that the PMSM system has many nonlinear properties such as multistability occurrence, the existence of the hidden attractors, the existence of two stable node-foci, and one saddle index-1. Recently, Saeed et al. [25] investigated the rub-impact force of an asymmetric nonlinear rotor system supported vertically. The authors integrated both linear position-velocity and cubic-velocity controllers simultaneously to control the system vibrations. The rub and impact forces between the pole legs and rotating shaft have been included in the derived model. The obtained results illustrated that the rub and impact forces can induce unbounded oscillation for the uncontrolled system. In addition, it is found that the proper tuning of the control parameters can prevent the rub and impact forces between the rotor and the legs of the poles.

Based on the above discussion, the dynamical behaviours of the asymmetric horizontally suspended rotor system with rub-impact force have not been investigated before as summarized in Table 1. Therefore, this work is dedicated to investigating the dynamical characteristics of an asymmetric nonlinear rotor system that is supported horizontally. The studied system is governed by two coupled second-order nonlinear differential equations having both cubic and quadratic nonlinearities which is excited by external and multiparametric forces. The linear proportional-derivative control algorithm is applied via four electromagnetic poles to control the nonlinear vibrations of the considered system. The rub and impact forces between stator and rotor are considered. The equations of motion of the controlled system are derived as a discontinuous nonlinear dynamical system. Due to the discontinuity of the derived model, the equations of motion have been analyzed in two stages. Firstly, the system is investigated analytically applying asymptotic methods when the rub and impact forces are neglected. The different response curves (i.e., spinning speed and eccentricity response curves) are obtained to explore the system's periodic oscillations and to determine the conditions at which the system may be subject to rub and/or impact forces. Secondly, utilizing the orbit plot, frequency spectrum, Poincare map, and bifurcation diagram, the whole system model is investigated including the rub and impact forces. The obtained results revealed that the studied system exhibits periodic oscillations only as long as the rub and impact forces do not occur. However, the occurrence of rub and impact forces is resulting in period-n, quasiperiodic, or chaotic oscillation for the rotor system. Moreover, the analytical and numerical simulations approved that the 
TABLE 1: Categorization of available literature on the asymmetric rotating shaft.

\begin{tabular}{|c|c|c|}
\hline Category & Subclassification & References \\
\hline \multirow[t]{3}{*}{ Dynamical analysis of the rotating shafts } & (i) Symmetric rotating shaft system & $\begin{array}{l}\text { (i) References } \\
{[8-10,15,26,27]}\end{array}$ \\
\hline & (ii) Asymmetric rotating shaft system & (ii) References $[1-7,12-14]$ \\
\hline & $\begin{array}{l}\text { (i) Symmetric rotating shaft with passive } \\
\text { control }\end{array}$ & (i) References [16-18] \\
\hline \multirow[t]{2}{*}{ Vibration control of the rotating shafts } & $\begin{array}{l}\text { (ii) Symmetric rotating shaft with active } \\
\text { control }\end{array}$ & (ii) References [19-22] \\
\hline & $\begin{array}{l}\text { (iii) Asymmetric rotating shaft system with } \\
\text { active control }\end{array}$ & (iii) References $[20,28]$ \\
\hline $\begin{array}{l}\text { Active vibration control of asymmetric rotating shafts } \\
\text { including the rub-impact forces }\end{array}$ & $\begin{array}{l}\text { (i) Nonlinear vertically supported rotor } \\
\text { (ii) Nonlinear horizontally supported rotor }\end{array}$ & $\begin{array}{l}\text { (i) Reference [25] } \\
\text { (ii) This work }\end{array}$ \\
\hline
\end{tabular}

proposed control strategy can eliminate the nonlinear behaviours of the studied system as well as prevent the rub and impact forces when the control gains are designed properly.

\section{Mathematical Formulation}

The dynamical model of the horizontally suspended rotor system shown in Figure 1(a) is given as follows [26-29]:

$$
\begin{aligned}
& m \ddot{u}_{1}+c_{1} \dot{u}_{1}+F_{U_{1}}=m e_{d} \omega^{2} \cos (\omega t+\gamma), \\
& m \ddot{u}_{2}+c_{2} \dot{u}_{2}+F_{U_{2}}=m e_{d} \omega^{2} \sin (\omega t+\gamma)-m g,
\end{aligned}
$$

where $m$ represents the disk mass, $c_{1}$ and $c_{2}$ denote the bearings damping in $U_{1}$ and $U_{2}$ directions, $F_{U_{1}}$ and $F_{U_{2}}$ are the restoring forces of the shaft in $U_{1}$ and $U_{2}$ directions, $e_{d}$ is the displacement between the geometric centre $G\left(u_{1}, u_{2}\right)$ of the rotor and its centroid is $e\left(u_{1 e}, u_{2 e}\right)$ as in Figure 1(b), $\omega$ represents the rotor angular speed, $m g$ is the disk weight, and $\gamma$ is the angle between $\overrightarrow{o \varsigma_{1}}$ axis and the eccentricity direction $\overrightarrow{G e}$. It is considered that the rotor system performs a planer vibration in the stationary $U_{1}-U_{2}$ frame, while $\varsigma_{1}-$ $\varsigma_{2}$ is a rotational frame that rotates with angular speed $\omega$. When the rotating shaft is displaced by small radial displacement $s=\sqrt{u_{1}^{2}+u_{2}^{2}}$ as shown in Figure 1(b), an exerted nonlinear restoring force is developed in $U_{1}$ and $U_{2}$ directions that can be expressed as follows [26-29]:

$$
\begin{aligned}
F_{u_{1}} & =\left(k_{1} s+k_{2} s^{3}\right) \cos \psi \\
& =k_{1} u_{1}+k_{2}\left(u_{1}^{2}+u_{2}^{2}\right) u_{1}, \\
F_{u_{2}} & =\left(k_{1} s+k_{2} s^{3}\right) \sin \psi \\
& =k_{1} u_{2}+k_{2}\left(u_{1}^{2}+u_{2}^{2}\right) u_{2},
\end{aligned}
$$

where $k_{1}$ is linear stiffness coefficient and $k_{2}$ is the nonlinear stiffness coefficient.

Substituting equations (2a) and (2b) into equations (1a) and (1b) yields

$$
\begin{gathered}
m \ddot{u}_{1}+c_{1} \dot{u}_{1}+k_{1} u_{1}+k_{2}\left(u_{1}^{2}+u_{2}^{2}\right) u_{1} \\
=m e_{d} \omega^{2} \cos (\omega t+\gamma), \\
m \ddot{u}_{2}+c_{2} \dot{u}_{2}+k_{1} u_{2}+k_{2}\left(u_{1}^{2}+u_{2}^{2}\right) u_{2} \\
=m e_{d} \omega^{2} \sin (\omega t+\gamma)-m g .
\end{gathered}
$$

Figure 1(a) shows that the disk weight $m g$ causes a small static deflection $u_{s}$ for the rotating shaft in $U_{2}$ direction. Accordingly, at static equilibrium, we have $\omega=\ddot{u}_{1}=\dot{u}_{1}=$ $u_{1}=\ddot{u}_{2}=\dot{u}_{2}=0.0$ and $u_{2}=u_{s}$. Substituting these conditions into equations ( $3 \mathrm{a})$ and $(3 \mathrm{~b})$ yields

$$
k_{1} u_{s}+k_{2} u_{s}^{3}=-m g
$$

To eliminate the constant $m g$ from equations (3a) and (3b), a new coordinate system $V_{1}-V_{2}$ is introduced such that $U_{1}=V_{1}$ and $U_{2}=u_{s}+V_{2}$. So, we have $\ddot{u}_{1}=\ddot{v}_{1}, \dot{u}_{1}=\dot{v}_{1}, u_{1}=v_{1}, \ddot{u}_{2}=\ddot{v}_{2}, \dot{u}_{2}=\dot{v}_{2}, u_{2}=u_{s}+v_{2}$.

Inserting the new variables into equations (3a) and (3b) considering equation (4) yields the following nonlinear dynamical system:

$$
\begin{aligned}
& m \ddot{v}_{1}+c_{1} \dot{v}_{1}+\left(k_{1}+k_{2} u_{s}^{2}\right) v_{1}+2 k_{2} u_{s} v_{1} v_{2}+k_{2}\left(v_{1}^{2}+v_{2}^{2}\right) v_{1} \\
& =m e_{d} \omega^{2} \cos (\omega t+\gamma),
\end{aligned}
$$

$$
\begin{aligned}
& m \ddot{v}_{2}+c_{2} \dot{v}_{2}+\left(k_{1}+3 k_{2} u_{s}^{2}\right) v_{2}+k_{2} u_{s}\left(v_{1}^{2}+3 v_{2}^{2}\right)+k_{2}\left(v_{1}^{2}+v_{2}^{2}\right) v_{2} \\
& =m e_{d} \omega^{2} \sin (\omega t+\gamma)
\end{aligned}
$$

For the asymmetric rotating machinery, the restoring force in $\varsigma_{1}$ and $\varsigma_{2}$ directions may be not symmetric due to the winding slots as in the case of 2-pole rotor generators [29] (see Figure 1(c)). Therefore, the equations of motions for such asymmetric machines can be obtained via modifying equations (5a) and (5b) as follows: 


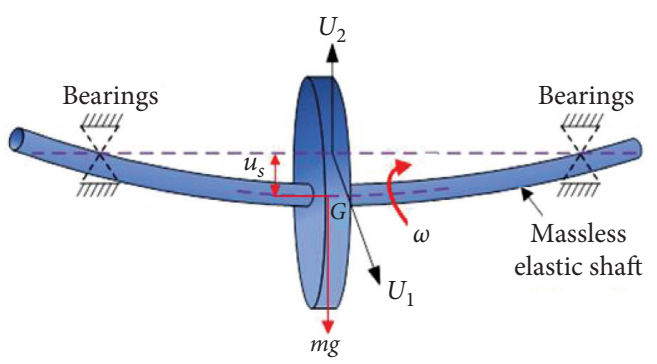

(a)

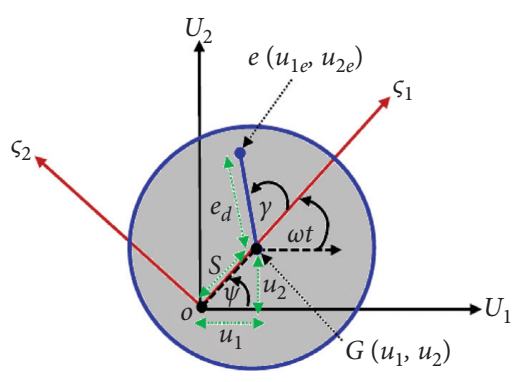

(b)

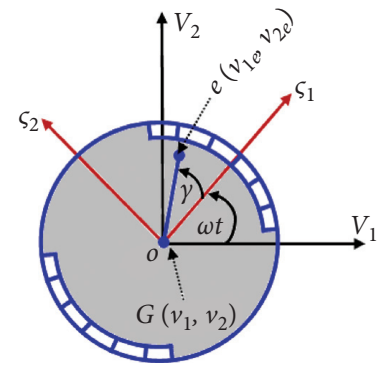

(c)

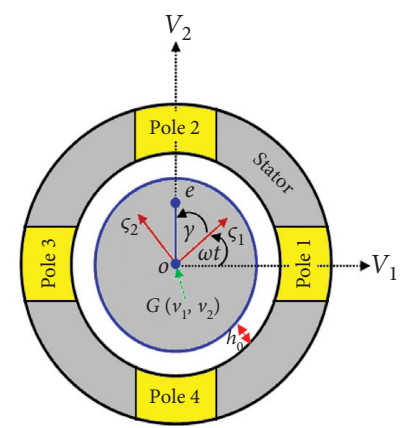

(d)

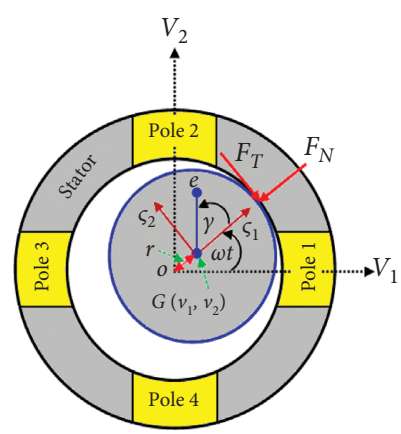

(e)

FIGURE 1: Horizontally supported asymmetric nonlinear rotor system: (a) Jeffcott rotor model, (b) symmetric disk, (c) asymmetric disk, (d) nondeflected rotating disk within the stator, and (e) deflected rotating disk within the stator.

$$
\begin{gathered}
m \ddot{v}_{1}+c_{1} \dot{v}_{1}+\left(k_{1}+k_{2} u_{s}^{2}\right) v_{1}+2 k_{2} u_{s} v_{1} v_{2}+k_{2}\left(v_{1}^{2}+v_{2}^{2}\right) v_{1}+\Delta F_{V_{1}}=m e_{d} \omega^{2} \cos (\omega t+\gamma), \\
m \ddot{v}_{2}+c_{2} \dot{v}_{2}+\left(k_{1}+3 k_{2} u_{s}^{2}\right) v_{2}+k_{2} u_{s}\left(v_{1}^{2}+3 v_{2}^{2}\right)+k_{2}\left(v_{1}^{2}+v_{2}^{2}\right) v_{2}+\Delta F_{V_{2}}=m e_{d} \omega^{2} \sin (\omega t+\gamma),
\end{gathered}
$$

where $\Delta F_{V_{1}}$ and $\Delta F_{V_{2}}$ represent the asymmetry of the restoring forces in $V_{1}$ and $V_{2}$ directions. It is considered that the difference between the restoring force in $\varsigma_{1}$ direction and in $\varsigma_{2}$ is $\Delta F$, where $\Delta F$ is given as follows [28]:

$$
\begin{aligned}
\Delta F= & \Delta k_{1} \varsigma_{1}+\Delta k_{2} \varsigma_{1}^{3} \\
= & \Delta k_{1}\left(v_{1} \cos (\omega t)+v_{2} \sin (\omega t)\right) \\
& +\Delta k_{2}\left(v_{1} \cos (\omega t)+v_{2} \sin (\omega t)\right)^{3},
\end{aligned}
$$

where $\Delta k_{1} \varsigma_{1}$ and $\Delta k_{2} \varsigma_{1}^{3}$ are the linear and nonlinear extra restoring forces in $\varsigma_{1}$ direction, respectively. By resolving $\Delta F$ in $V_{1}$ and $V_{2}$ directions, we can express their components $\Delta F_{V_{1}}$ and $\Delta F_{V_{2}}$ as follows:

$$
\begin{aligned}
\Delta F_{V_{1}}= & \Delta F \cos (\omega t)=\frac{1}{2} \Delta k_{1} v_{1}+\frac{3}{8} \Delta k_{2} v_{1}^{3}+\frac{3}{8} \Delta k_{2} v_{1} v_{2}^{2} \\
& +\frac{1}{2} \Delta k_{1} v_{1} \cos (2 \omega t)+\frac{1}{2} \Delta k_{1} v_{2} \sin (2 \omega t)+\frac{1}{2} \Delta k_{2} v_{1}^{3} \cos (2 \omega t) \\
& +\frac{3}{4} \Delta k_{2} v_{1}^{2} v_{2} \sin (2 \omega t)+\frac{1}{4} \Delta k_{2} v_{2}^{3} \sin (2 \omega t)+\frac{1}{8} \Delta k_{2} v_{1}^{3} \cos (4 \omega t) \\
& -\frac{1}{8} \Delta k_{2} v_{2}^{3} \sin (4 \omega t)+\frac{3}{8} \Delta k_{2} v_{1}^{2} v_{2} \sin (4 \omega t)-\frac{3}{8} \Delta k_{2} v_{1} v_{2}^{2} \cos (4 \omega t)
\end{aligned}
$$




$$
\begin{aligned}
\Delta F_{V_{2}}= & \Delta F \sin (\omega t)=\frac{1}{2} \Delta k_{1} v_{2}+\frac{3}{8} \Delta k_{2} v_{2}^{3}+\frac{3}{8} \Delta k_{2} v_{1}^{2} v_{2} \\
& -\frac{1}{2} \Delta k_{1} v_{2} \cos (2 \omega t)+\frac{1}{2} \Delta k_{1} v_{1} \sin (2 \omega t)-\frac{1}{2} \Delta k_{2} v_{2}^{3} \cos (2 \omega t) \\
& +\frac{3}{4} \Delta k_{2} v_{1} v_{2}^{2} \sin (2 \omega t)+\frac{1}{4} \Delta k_{2} v_{1}^{3} \sin (2 \omega t)+\frac{1}{8} \Delta k_{2} v_{2}^{3} \cos (4 \omega t) \\
& +\frac{1}{8} \Delta k_{2} v_{1}^{3} \sin (4 \omega t)-\frac{3}{8} \Delta k_{2} v_{1} v_{2}^{2} \sin (4 \omega t)-\frac{3}{8} \Delta k_{2} v_{1}^{2} v_{2} \cos (4 \omega t)
\end{aligned}
$$

Inserting equations (8a) and (8b) into equations (6a) and

(6b), we have

$$
\begin{aligned}
m \ddot{v}_{1}+ & c_{1} \dot{v}_{1}+\left(k_{1}+\frac{1}{2} \Delta k_{1}+k_{2} u_{s}^{2}\right) v_{1}+2 k_{2} u_{s} v_{1} v_{2}+\left(k_{2}+\frac{3}{8} \Delta k_{2}\right)\left(v_{1}^{2}+v_{2}^{2}\right) v_{1} \\
= & m e_{d} \omega^{2} \cos (\omega t+\gamma)-\frac{1}{2} \Delta k_{1} v_{1} \cos (2 \omega t)-\frac{1}{2} \Delta k_{1} v_{2} \sin (2 \omega t) \\
& -\frac{1}{2} \Delta k_{2} v_{1}^{3} \cos (2 \omega t)-\frac{3}{4} \Delta k_{2} v_{1}^{2} v_{2} \sin (2 \omega t)-\frac{1}{4} \Delta k_{2} v_{2}^{3} \sin (2 \omega t) \\
& -\frac{1}{8} \Delta k_{2} v_{1}^{3} \cos (4 \omega t)+\frac{1}{8} \Delta k_{2} v_{2}^{3} \sin (4 \omega t) \\
& -\frac{3}{8} \Delta k_{2} v_{1}^{2} v_{2} \sin (4 \omega t)+\frac{3}{8} \Delta k_{2} v_{1} v_{2}^{2} \cos (4 \omega t)+F_{11}+F_{12}, \\
m \ddot{v}_{2}+ & c_{2} \dot{v}_{2}+\left(k_{1}+\frac{1}{2} \Delta k_{1}+3 k_{2} u_{s}^{2}\right) v_{2}+k_{2} u_{s}\left(v_{1}^{2}+3 v_{2}^{2}\right)+\left(k_{2}+\frac{3}{8} \Delta k_{2}\right)\left(v_{1}^{2}+v_{2}^{2}\right) v_{2} \\
= & m e_{d} \omega^{2} \sin (\omega t+\gamma)+\frac{1}{2} \Delta k_{1} v_{2} \cos (2 \omega t)-\frac{1}{2} \Delta k_{1} v_{1} \sin (2 \omega t)+\frac{1}{2} \Delta k_{2} v_{2}^{3} \cos (2 \omega t) \\
& -\frac{3}{4} \Delta k_{2} v_{1} v_{2}^{2} \sin (2 \omega t)-\frac{1}{4} \Delta k_{2} v_{1}^{3} \sin (2 \omega t)-\frac{1}{8} \Delta k_{2} v_{2}^{3} \cos (4 \omega t)-\frac{1}{8} \Delta k_{2} v_{1}^{3} \sin (4 \omega t) \\
& +\frac{3}{8} \Delta k_{2} v_{1} v_{2}^{2} \sin (4 \omega t)+\frac{3}{8} \Delta k_{2} v_{1}^{2} v_{2} \cos (4 \omega t)+F_{21}+F_{22} .
\end{aligned}
$$

the above nonlinear dynamical system governs the lateral vibrations $V_{1}$ and $V_{2}$ of the asymmetric horizontally supported rotating shaft. The forces $F_{11}$ and $F_{21}$ have been added to equations (9a) and (9b) as control forces generated via electromagnetic poles as shown in Figure 1(d) to control the system vibrations in $V_{1}$ and $V_{2}$ directions, while the forces $F_{12}$ and $F_{22}$ are induced in $V_{1}$ and $V_{2}$ directions due to the rub and impact forces $\left(F_{N}\right.$ and $\left.F_{T}\right)$ that occur between the rotor and stator when the applied control forces $\left(F_{11}\right.$ and $\left.F_{21}\right)$ fail to prevent the contact of the rotating shaft and the stator as illustrated in Figure 1(e). Depending on the proposed electromagnetic poles (see Figures 1(d) and 1(e)), the forces $F_{11}$ and $F_{21}$ are given as follows [30]:

$$
\begin{aligned}
F_{11} & =F\left(v_{1}, i_{v_{1}}\right) \\
& =\xi\left[\frac{\left(I_{0}+i_{v_{1}}\right)^{2}}{\left(h_{0}+v_{1}\right)^{2}}-\frac{\left(I_{0}-i_{v_{1}}\right)^{2}}{\left(h_{0}-v_{1}\right)^{2}}\right], \\
F_{21} & =F\left(v_{2}, i_{v_{2}}\right) \\
& =\xi\left[\frac{\left(I_{0}+i_{v_{2}}\right)^{2}}{\left(h_{0}+v_{2}\right)^{2}}-\frac{\left(I_{0}-i_{v_{2}}\right)^{2}}{\left(h_{0}-v_{2}\right)^{2}}\right],
\end{aligned}
$$

where pole 1 and pole 3 apply force $F_{11}$, while pole 2 and pole 4 apply force $F_{21}$, $\xi$ denotes the magnetic force constant, $i_{v_{1}}$ and $i_{v_{2}}$ are the control currents, $I_{0}$ is the magnetizing current, and $h_{0}$ denotes the clearance between the stator and the 
rotor (i.e., $h_{0}$ is the static air gap as shown in Figure $1(\mathrm{~d})$ ). Expanding equations (10a) and (10b) in terms of $i_{v_{1}}, i_{v_{2}}, v_{1}$, and $v_{2}$ up to the third-order approximation using Maclaurin series yields

$$
\begin{aligned}
& F_{11}=\frac{4 \xi}{h_{0}^{5}}\left(-h_{0}^{3} I_{0} i_{v_{1}}+h_{0}^{2} I_{0}^{2} v_{1}+2 I_{0}^{2} v_{1}^{3}-3 h_{0} I_{0} i_{v_{1}} v_{1}^{2}+h_{0}^{2} i_{v_{1}}^{2} v_{1}\right), \\
& F_{21}=\frac{4 \xi}{h_{0}^{5}}\left(-h_{0}^{3} I_{0} i_{v_{2}}+h_{0}^{2} I_{0}^{2} v_{2}+2 I_{0}^{2} v_{2}^{3}-3 h_{0} I_{0} i_{v_{2}} v_{2}^{2}+h_{0}^{2} i_{v_{2}}^{2} v_{2}\right),
\end{aligned}
$$

where $i_{v_{1}}$ and $i_{v_{2}}$ are selected to be a function of both the disk displacements $\left(v_{1}\right.$ and $\left.v_{2}\right)$ and disk velocities $\left(\dot{v}_{1}\right.$ and $\left.\dot{v}_{2}\right)$ as follows:

$$
\begin{aligned}
& i_{v_{1}}=k_{p} v_{1}+k_{d} \dot{v}_{1}, \\
& i_{v_{2}}=k_{p} v_{2}+k_{d} \dot{v}_{2},
\end{aligned}
$$

where $k_{p}$ and $k_{d}$ are the proportional and derivative control gains, respectively. The considered system may be subject to rub and impact forces as illustrated in Figure 1(e). The rub and/or impact forces occur when the radial displacement

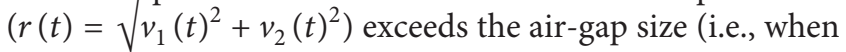
$\left.(t) \geq h_{0}\right)$. The impact force $F_{N}$ and rub force $F_{T}$ develop at the stator-rotor interface as depicted in Figure 1(e). Accordingly, the rub can be expressed as follows [25]:

$$
\begin{aligned}
F_{N} & =k_{c}\left(r-h_{0}\right) H\left(r-h_{0}\right), \\
F_{T} & =\mu_{d} F_{N} \\
& =\mu_{d} k_{c}\left(r-h_{0}\right) H\left(r-h_{0}\right),
\end{aligned}
$$

where $k_{c}$ denotes the impact stiffness coefficient, $\mu_{d}$ represents the dynamic frictional coefficient between the rotating disk and the pole leg, and $H$ denotes the Heaviside function. Resolving the forces $F_{N}$ and $F_{T}$ into their components in $V_{1}$ and $V_{2}$ directions and considering $\cos (\omega t)=\left(v_{1} / r\right)$, $\sin (\omega t)=\left(v_{2} / r\right)$, we get

$$
\begin{aligned}
F_{21} & =F_{T} \sin (\omega t)-F_{N} \cos (\omega t) \\
& =\frac{k_{c}}{r}\left(r-h_{0}\right)\left(\mu_{d} v_{2}-v_{1}\right) H\left(r-h_{0}\right), \\
F_{22} & =-F_{T} \cos (\omega t)-F_{N} \sin (\omega t) \\
& =-\frac{k_{c}}{r}\left(r-h_{0}\right)\left(\mu_{d} v_{1}+v_{2}\right) H\left(r-h_{0}\right) .
\end{aligned}
$$

Inserting equations (11), (12), (15), and (16) into equations (9a) and (9b) taking into account equation (13) with introducing the dimensionless variables $\tau=\omega_{n} t$, $z_{1}=\left(v_{1} / h_{0}\right), z_{2}=\left(v_{2} / h_{0}\right), R=\left(r / h_{0}\right)=\sqrt{z_{1}^{2}+z_{2}^{2}}$, we get the following dimensionless equations:

$$
\begin{aligned}
\ddot{z}_{1}+\mu_{1} \dot{z}_{1}+\omega_{1}^{2} z_{1}+2 \eta z_{1} z_{2}+\beta\left(z_{1}^{2}+z_{2}^{2}\right) z_{1}= & f \Omega^{2} \cos (\Omega \tau+\gamma)-\frac{1}{2}\left(\delta_{1}+\delta_{2} z_{1}^{2}\right) z_{1} \cos (2 \Omega \tau) \\
& -\frac{1}{2}\left(\delta_{1}+\frac{1}{2} \delta_{2}\left(3 z_{1}^{2}+z_{2}^{2}\right)\right) z_{2} \sin (2 \Omega \tau)+\frac{1}{8} \delta_{2}\left(3 z_{1} z_{2}^{2}-z_{1}^{3}\right) \cos (4 \Omega \tau) \\
& +\frac{1}{8} \delta_{2}\left(z_{2}^{3}-3 z_{1}^{2} z_{2}\right) \sin (4 \Omega \tau) \\
& -\left(\alpha_{1} z_{1}+\alpha_{2} \dot{z}_{1}+\alpha_{3} z_{1}^{3}+\alpha_{4} z_{1}^{2} \dot{z}_{1}+\alpha_{5} z_{1} \dot{z}_{1}^{2}\right)+\frac{\delta_{3}}{R}(R-1)\left(\mu_{d} z_{2}-z_{1}\right) H(R-1),
\end{aligned}
$$

$\ddot{z}_{2}+\mu_{2} \dot{z}_{2}+\omega_{2}^{2} z_{2}+\eta\left(z_{1}^{2}+3 z_{2}^{2}\right)+\beta\left(z_{1}^{2}+z_{2}^{2}\right) z_{2}=f \Omega^{2} \sin (\Omega \tau+\gamma)+\frac{1}{2}\left(\delta_{1}+\delta_{2} z_{2}^{2}\right) z_{2} \cos (2 \Omega \tau)$

$$
\begin{aligned}
& -\frac{1}{2}\left(\delta_{1}+\frac{1}{2} \delta_{2}\left(3 z_{2}^{2}+z_{1}^{2}\right)\right) z_{1} \sin (2 \Omega \tau)+\frac{1}{8} \delta_{2}\left(3 z_{1}^{2} z_{2}-z_{2}^{3}\right) \cos (4 \Omega \tau) \\
& +\frac{1}{8} \delta_{2}\left(3 z_{1} z_{2}^{2}-z_{1}^{3}\right) \sin (4 \Omega \tau) \\
& -\left(\alpha_{1} z_{2}+\alpha_{2} \dot{z}_{2}+\alpha_{3} z_{2}^{3}+\alpha_{4} z_{2}^{2} \dot{z}_{2}+\alpha_{5} z_{2} \dot{z}_{2}^{2}\right)-\frac{\delta_{3}}{R}(R-1)\left(\mu_{d} z_{1}+z_{2}\right) H(R-1),
\end{aligned}
$$


where $\mu_{1}=\left(c_{1} / \sqrt{k_{1} m}\right), \quad \mu_{2}=\left(c_{2} / \sqrt{k_{1} m}\right), \omega_{n}^{2}=\left(k_{1} / m\right)=$ $\left(\xi I_{0}^{2} / m h_{0}\right), \Omega=\left(\omega / \omega_{n}\right), f=\left(e_{d} / h_{0}\right), \quad \lambda=\left(u_{s} / h_{0}\right), \rho=$ $\left(h_{0}^{2} k_{2} / k_{1}\right), \eta=\lambda \rho, \delta_{1}=\left(\Delta k_{1} / k_{1}\right), \delta_{2}=\left(h_{0}^{2} \Delta k_{2} / k_{1}\right), \quad \delta_{3}=$ $\left(k_{c} / k_{1}\right), \omega_{1}=\sqrt{1+\lambda^{2} \rho+(1 / 2) \delta_{1}}$,

$\omega_{2}=\sqrt{1+3 \lambda^{2} \rho+(1 / 2) \delta_{1}}, \beta=\rho+\quad(3 / 8) \delta_{2}, p=\left(h_{0} / I_{0}\right)$ $k_{p}, d=\left(h_{0} \omega_{n} / I_{0}\right) k_{d}, \quad \alpha_{1}=p-1, \alpha_{2}=d, \alpha_{3}=-2+$ $3 p-p^{2}, \alpha_{4}=3 d-2 p d, \alpha_{5}=-d^{2}$.

Equations (17a) and (17b) govern the whole system dynamics. As the studied system is governed by a discontinuous dynamical model, equations (17a) and (17b) are analyzed in two steps. In the first step, the rub-impact force is neglected via setting $\delta_{3}=\left(k_{c} / k_{1}\right)=0.0$. The corresponding continuous dynamical system is investigated analytically using the perturbation method to obtain the amplitude-phase modulation equations as given in Section 3. Based on the obtained amplitude-phase modulation equations (when $\delta_{3}=0.0$ ), the conditions at which the whole system can exhibit rub-impact force can be predicted via plotting the different response curves. In the second step of the analysis, the dynamical behaviours of the whole system (i.e., equations $(17 \mathrm{a})$ and $(17 \mathrm{~b})$ when $\delta_{3} \neq 0.0$ ) are simulated using the bifurcation diagrams, Poincare map, frequency spectrum, and whirling orbits.

\section{Amplitude-Phase Modulating Equations When $\boldsymbol{\delta}_{3}==0.0$}

Within this section, a second-order approximate solution to equations (17a) and (17b) when $\delta_{3}=0.0$ can be sought using the multiple scales perturbation method as follows [31]:

$$
\begin{aligned}
z_{1}(\tau)= & z_{11}\left(T_{0}, T_{1}, T_{2}\right)+\varepsilon z_{12}\left(T_{0}, T_{1}, T_{2}\right) \\
& +\varepsilon^{2} z_{13}\left(T_{0}, T_{1}, T_{2}\right)+O\left(\varepsilon^{3}\right), \\
z_{2}(\tau)= & z_{21}\left(T_{0}, T_{1}, T_{2}\right)+\varepsilon z_{22}\left(T_{0}, T_{1}, T_{2}\right) \\
& +\varepsilon^{2} z_{23}\left(T_{0}, T_{1}, T_{2}\right)+O\left(\varepsilon^{3}\right),
\end{aligned}
$$

where $\varepsilon$ is a book-keeping perturbation parameter and $T_{j}=\varepsilon^{j} \tau, j=0,1,2$. In terms of $T_{0}, T_{1}$, and $T_{2}$, the derivatives $(d / d \tau)$ can be expressed as

$$
\begin{aligned}
\frac{\mathrm{d}}{\mathrm{d} \tau} & =D_{0}+\varepsilon D_{1}+\varepsilon^{2} D_{2}, \\
\frac{\mathrm{d}^{2}}{\mathrm{~d} \tau^{2}} & =D_{0}^{2}+2 \varepsilon D_{0} D_{1}+\varepsilon^{2}\left(D_{1}^{2}+2 D_{2} D_{0}\right), \\
D_{j} & =\frac{\partial}{\partial T_{j}}, \quad j=0,1,2 .
\end{aligned}
$$

The system parameters are scaled such that

$$
\begin{aligned}
\mu_{1} & =\varepsilon^{2} \widehat{\mu}_{1}, \\
\mu_{2} & =\varepsilon^{2} \widehat{\mu}_{2}, \\
\beta & =\varepsilon^{2} \widehat{\beta}, \\
\eta & =\varepsilon \widehat{\eta}, \\
f & =\varepsilon \widehat{f}, \\
\delta_{1} & =\varepsilon^{2} \widehat{\delta}_{1}, \\
\delta_{2} & =\varepsilon^{2} \widehat{\delta}_{2}, \\
\alpha_{n} & =\varepsilon^{2} \widehat{\alpha}_{n}, \quad n=1,2, \ldots, 5 .
\end{aligned}
$$

Substituting equations (18a) to (20) into equations (17a) and $(17 \mathrm{~b})$ and then equating coefficients of the same power of $\varepsilon$, we get

$$
O\left(\varepsilon^{0}\right) \text { : }
$$

$$
\begin{aligned}
& \left(D_{0}^{2}+\omega_{1}^{2}\right) z_{11}=0 \\
& \left(D_{0}^{2}+\omega_{2}^{2}\right) z_{21}=0 .
\end{aligned}
$$

$O\left(\varepsilon^{1}\right)$

$$
\begin{gathered}
\left(D_{0}^{2}+\omega_{1}^{2}\right) z_{12}=-2 D_{0} D_{1} z_{11}-2 \widehat{\eta} z_{21} z_{11}+\widehat{f} \Omega^{2} \cos \left(\Omega T_{0}+\gamma\right) \\
\left(D_{0}^{2}+\omega_{2}^{2}\right) z_{22}=-2 D_{0} D_{1} z_{21}-\widehat{\eta} z_{11}^{2}-3 \widehat{\eta} z_{21}^{2}+\widehat{f} \Omega^{2} \sin \left(\Omega T_{0}+\gamma\right) .
\end{gathered}
$$

$O\left(\varepsilon^{2}\right):$ 


$$
\begin{aligned}
\left(D_{0}^{2}+\omega_{1}^{2}\right) z_{13}= & -2 D_{0} D_{1} z_{12}-\left(D_{1}^{2}+2 D_{2} D_{0}\right) z_{11}-\widehat{\mu}_{1} D_{0} z_{11}-2 \widehat{\eta} z_{21} z_{12}-2 \widehat{\eta} z_{22} z_{11}-\widehat{\beta} z_{21}^{2} z_{11}-\widehat{\beta} z_{11}^{3}-\frac{1}{2} \widehat{\delta}_{1} z_{11} \cos \left(2 \Omega T_{0}\right) \\
& -\frac{1}{2} \widehat{\delta}_{1} z_{21} \sin \left(2 \Omega T_{0}\right)-\frac{1}{2} \widehat{\delta}_{2} z_{11}^{3} \cos \left(2 \Omega T_{0}\right)-\frac{1}{4} \widehat{\delta}_{2}\left(3 z_{11}^{2} z_{21}+z_{21}^{3}\right) \sin \left(2 \Omega T_{0}\right)+\frac{1}{8} \widehat{\delta}_{2}\left(3 z_{11} z_{21}^{2}-z_{11}^{3}\right) \cos \left(4 \Omega T_{0}\right) \\
& +\frac{1}{8} \widehat{\delta}_{2}\left(z_{21}^{3}-3 z_{11}^{2} z_{21}\right) \sin \left(4 \Omega T_{0}\right)-\widehat{\alpha}_{1} z_{11}-\widehat{\alpha}_{2} D_{0} z_{11}-\widehat{\alpha}_{3} z_{11}^{3}-\widehat{\alpha}_{4} z_{11}^{2} D_{0} z_{11}-\widehat{\alpha}_{5} z_{11}\left(D_{0} z_{11}\right)^{2} \\
\left(D_{0}^{2}+\omega_{2}^{2}\right) z_{23}= & -2 D_{0} D_{1} z_{22}-\left(D_{1}^{2}+2 D_{2} D_{0}\right) z_{21}-\widehat{\mu}_{2} D_{0} z_{21}-2 \widehat{\eta} z_{11} z_{12}-6 \widehat{\eta} z_{21} z_{22}-\widehat{\beta} z_{21} z_{11}^{2} \\
& -\widehat{\beta} z_{21}^{3}-\frac{1}{2} \widehat{\delta}_{1} z_{11} \sin \left(2 \Omega T_{0}\right)+\frac{1}{2} \widehat{\delta}_{1} z_{21} \cos \left(2 \Omega T_{0}\right)+\frac{1}{2} \widehat{\delta}_{2} z_{21}^{3} \cos \left(2 \Omega T_{0}\right)-\frac{1}{4} \widehat{\delta}_{2}\left(3 z_{11} z_{21}^{2}+z_{11}^{3}\right) \sin \left(2 \Omega T_{0}\right) \\
& +\frac{1}{8} \widehat{\delta}_{2}\left(3 z_{11}^{2} z_{21}-z_{21}^{3}\right) \cos \left(4 \Omega T_{0}\right)+\frac{1}{8} \widehat{\delta}_{2}\left(3 z_{11} z_{21}^{2}-z_{11}^{3}\right) \sin \left(4 \Omega T_{0}\right)-\widehat{\alpha}_{1} z_{21}-\widehat{\alpha}_{2} D_{0} z_{21}-\widehat{\alpha}_{3} z_{21}^{3} \\
& -\widehat{\alpha}_{4} z_{21}^{2} D_{0} z_{21}-\widehat{\alpha}_{5} z_{21}\left(D_{0} z_{21}\right)^{2} .
\end{aligned}
$$

The solution of equations (21a) and (21b) can be expressed as

$$
\begin{aligned}
& z_{11}\left(T_{0}, T_{1}, T_{2}\right)=\chi_{1}\left(T_{1}, T_{2}\right) e^{i \omega_{1} T_{0}}+\bar{\chi}_{1}\left(T_{1}, T_{2}\right) e^{-i \omega_{1} T_{0}} \\
& z_{21}\left(T_{0}, T_{1}, T_{2}\right)=\chi_{2}\left(T_{1}, T_{2}\right) e^{i \omega_{2} T_{0}}+\bar{\chi}_{2}\left(T_{1}, T_{2}\right) e^{-i \omega_{2} T_{0}},
\end{aligned}
$$

where $\chi_{1}\left(T_{1}, T_{2}\right)$ and $\chi_{2}\left(T_{1}, T_{2}\right)$ are unknown functions of the time scales $T_{1}$ and $T_{2}$. Inserting equations (24a) and (24b) into equations (22a) and (22b), we have

$$
\begin{gathered}
\left(D_{0}^{2}+\omega_{1}^{2}\right) z_{12}=-2 i \omega_{1} D_{1} \chi_{1} e^{i \omega_{1} T_{0}}-2 \widehat{\eta} \chi_{1} \chi_{2} e^{i\left(\omega_{1}+\omega_{2}\right) T_{0}}-2 \widehat{\eta} \chi_{1} \bar{\chi}_{2} e^{i\left(\omega_{1}-\omega_{2}\right) T_{0}}+\frac{\widehat{f} \Omega^{2}}{2} e^{i\left(\Omega T_{0}+\gamma\right)}+c c \\
\left(D_{0}^{2}+\omega_{2}^{2}\right) z_{22}=-2 i \omega_{2} D_{1} \chi_{2} e^{i \omega_{2} T_{0}}-\widehat{\eta}\left(\chi_{1}^{2} e^{2 i \omega_{1} T_{0}}+\chi_{1} \bar{\chi}_{1}\right)-3 \widehat{\eta}\left(\chi_{2}^{2} e^{2 i \omega_{2} T_{0}}+\chi_{2} \bar{\chi}_{2}\right)-\frac{i \widehat{f} \Omega^{2}}{2} e^{i\left(\Omega T_{0}+\gamma\right)}+c c
\end{gathered}
$$

where $c c$ denotes the complex conjugate term. The primary resonance and internal resonance cases (i.e., $\Omega=\omega_{1}=\omega_{2}$ ) are investigated simultaneously in this work, where the detuning parameters $\sigma$ and $\sigma_{1}$ are utilized to describe the closeness of $\Omega$ and $\omega_{2}$ to $\omega_{1}$ as

$$
\begin{aligned}
\Omega & =\omega_{1}+\sigma \\
& =\omega_{1}+\varepsilon \widehat{\sigma}, \\
\omega_{2} & =\omega_{1}+\sigma_{1} \\
& =\omega_{1}+\varepsilon \widehat{\sigma}_{1} .
\end{aligned}
$$

Substituting equation (26) into equations (25a) and (25b) yields

$$
\begin{gathered}
\left(D_{0}^{2}+\omega_{1}^{2}\right) z_{12}=2\left(-2 i \omega_{1} D_{1} \chi_{1}+\frac{\widehat{f} \Omega^{2}}{2} e^{i\left(\widehat{\sigma} T_{1}+\gamma\right)}\right) e^{i \omega_{1} T_{0}}-2 \widehat{\eta} \chi_{1} \chi_{2} e^{i\left(\omega_{1}+\omega_{2}\right) T_{0}}-2 \widehat{\eta} \chi_{1} \bar{\chi}_{2} e^{i\left(\omega_{1}-\omega_{2}\right) T_{0}}+c c \\
\left(D_{0}^{2}+\omega_{2}^{2}\right) z_{22}=\left(-2 i \omega_{2} D_{1} \chi_{2}+\frac{\widehat{f} \Omega^{2}}{2 i} e^{i\left(\left(\widehat{\sigma}-\widehat{\sigma}_{1}\right) T_{1}+\gamma\right)}\right) e^{i \omega_{2} T_{0}}-\widehat{\eta}\left(\chi_{1}^{2} e^{2 i \omega_{1} T_{0}}+\chi_{1} \bar{\chi}_{1}\right)-3 \widehat{\eta}\left(\chi_{2}^{2} e^{2 i \omega_{2} T_{0}}+\chi_{2} \bar{\chi}_{2}\right)+c c
\end{gathered}
$$


The solvability conditions of equations (27a) and (27b) are

$$
\begin{aligned}
2 i \omega_{1} D_{1} \chi_{1} & =\frac{\hat{f} \Omega^{2}}{2} e^{i\left(\widehat{\sigma} T_{1}+\gamma\right)}, \\
2 i \omega_{2} D_{1} \chi_{2} & =-\frac{i \widehat{f} \Omega^{2}}{2} e^{i\left(\left(\widehat{\sigma}-\widehat{\sigma}_{1}\right) T_{1}+\gamma\right)} .
\end{aligned}
$$

According to equation (28), the solution of equations (27a) and (27b) can be expressed as follows:

$$
\begin{gathered}
z_{12}\left(T_{0}, T_{1}, T_{2}\right)=\frac{2 \widehat{\eta}}{\omega_{2}\left(2 \omega_{1}+\omega_{2}\right)} \chi_{1} \chi_{2} e^{i\left(\omega_{1}+\omega_{2}\right) T_{0}}+\frac{2 \widehat{\eta}}{\omega_{2}\left(\omega_{2}-2 \omega_{1}\right)} \chi_{1} \bar{\chi}_{2} e^{i\left(\omega_{1}-\omega_{2}\right) T_{0}}+c c, \\
z_{22}\left(T_{0}, T_{1}, T_{2}\right)=\frac{\widehat{\eta}}{4 \omega_{1}^{2}-\omega_{2}^{2}} \chi_{1}^{2} e^{2 i \omega_{1} T_{0}}+\frac{\widehat{\eta}}{\omega_{2}^{2}} \chi_{2}^{2} e^{2 i \omega_{2} T_{0}}-\frac{\widehat{\eta} \chi_{1} \bar{\chi}_{1}}{\omega_{2}^{2}}-\frac{3 \widehat{\delta} \chi_{2} \bar{\chi}_{2}}{\omega_{2}^{2}}+c c .
\end{gathered}
$$

Substituting equations (24a), (24b), (29a), and (29b) into the right-hand side of equations (23a) and (23b), one finds the following solvability conditions of equations (23a) and (23b):

$$
\begin{aligned}
2 i \omega_{1} D_{2} \chi_{1}= & -D_{1}^{2} \chi_{1}-\left(i \omega_{1} \widehat{\mu}_{1}+\widehat{\alpha}_{1}+i \omega_{1} \widehat{\alpha}_{2}\right) \chi_{1}+\left(\frac{4 \widehat{\eta}^{2}}{\omega_{2}^{2}}-\frac{2 \widehat{\eta}^{2}}{4 \omega_{1}^{2}-\omega_{2}^{2}}-3 \widehat{\beta}-3 \widehat{\alpha}_{3}-i \omega_{1} \widehat{\alpha}_{4}-\omega_{1}^{2} \widehat{\alpha}_{5}\right) \chi_{1}^{2} \bar{\chi}_{1} \\
& +\left(\frac{12 \widehat{\eta}^{2}}{\omega_{2}^{2}}-\frac{4 \widehat{\eta}^{2}}{\omega_{2}\left(\omega_{2}-2 \omega_{1}\right)}-\frac{4 \widehat{\eta}^{2}}{\omega_{2}\left(2 \omega_{1}+\omega_{2}\right)}-2 \widehat{\beta}\right) \chi_{1} \chi_{2} \bar{\chi}_{2}-\left(\frac{2 \widehat{\eta}^{2}}{\omega_{2}^{2}}+\frac{4 \widehat{\eta}^{2}}{\omega_{2}\left(\omega_{2}-2 \omega_{1}\right)}+\widehat{\beta}\right) \bar{\chi}_{1} \chi_{2}^{2} e^{2 i \widehat{\sigma}_{1} T_{1}}-\frac{1}{4} \widehat{\delta}_{1} \bar{\chi}_{1} e^{2 \hat{\sigma} T_{1}} \\
& +\frac{i}{4} \widehat{\delta}_{1} \bar{\chi}_{2} e^{i\left(2 \widehat{\sigma}-\widehat{\sigma}_{1}\right) T_{1}}-\frac{3}{4} \widehat{\delta}_{2} \chi_{1} \bar{\chi}_{1}^{2} e^{2 \hat{\sigma} T_{1}}-\frac{1}{4} \widehat{\delta}_{2} \chi_{1}^{3} e^{-2 \hat{i} T_{1}}+\frac{3 i}{8} \widehat{\delta}_{2} \bar{\chi}_{1}^{2} \chi_{2} e^{i\left(2 \widehat{\sigma}-\widehat{\sigma}_{1}\right) T_{1}} \\
& -\frac{3 i}{8} \widehat{\delta}_{2} \chi_{1}^{2} \chi_{2} e^{i\left(-2 \widehat{\sigma}-\widehat{\sigma}_{1}\right) T_{1}}+\frac{3 i}{4} \widehat{\delta}_{2} \chi_{1} \bar{\chi}_{1} \bar{\chi}_{2} e^{i\left(2 \widehat{\sigma}-\widehat{\sigma}_{1}\right) T_{1}} \\
& +\frac{3 i}{8} \widehat{\delta}_{2} \chi_{2} \bar{\chi}_{2}^{2} e^{i\left(2 \widehat{\sigma}-\widehat{\sigma}_{1}\right) T_{1}}-\frac{i}{8} \widehat{\delta}_{2} \chi_{2}^{3} e^{i\left(-2 \widehat{\sigma}-3 \widehat{\sigma}_{1}\right) T_{1}}+\frac{3}{16} \widehat{\delta}_{2} \bar{\chi}_{1} \bar{\chi}_{2}^{2} e^{i\left(4 \widehat{\sigma}-2 \widehat{\sigma}_{1}\right) T_{1}}-\frac{1}{16} \widehat{\delta}_{2} \bar{\chi}_{1}^{3} e^{4 \widehat{i} T_{1}} \\
& -\frac{i}{16} \widehat{\delta}_{2} \bar{\chi}_{2}^{3} e^{i\left(4 \widehat{\sigma}-3 \widehat{\sigma}_{1}\right) T_{1}}+\frac{3 i}{16} \widehat{\delta}_{2} \bar{\chi}_{1}^{2} \bar{\chi}_{2} e^{i\left(\widehat{4} \widehat{\sigma}-\widehat{\sigma}_{1}\right) T_{1}}
\end{aligned}
$$

$$
\begin{aligned}
2 i \omega_{2} D_{2} \chi_{2}= & -D_{1}^{2} \chi_{2}-\left(i \omega_{2} \widehat{\mu}_{2}+\widehat{\alpha}_{1}+i \omega_{2} \widehat{\alpha}_{2}\right) \chi_{2}+\left(\frac{30 \widehat{\eta}^{2}}{\omega_{2}^{2}}-3 \widehat{\beta}-3 \widehat{\alpha}_{3}-i \omega_{2} \widehat{\alpha}_{4}-\omega_{2}^{2} \widehat{\alpha}_{5}\right) \chi_{2}^{2} \bar{\chi}_{2} \\
& -\left(\frac{4 \widehat{\eta}^{2}}{\omega_{2}\left(2 \omega_{1}+\omega_{2}\right)}+\frac{4 \widehat{\eta}^{2}}{\omega_{2}\left(\omega_{2}-2 \omega_{1}\right)}-\frac{12 \widehat{\eta}^{2}}{\omega_{2}^{2}}+2 \widehat{\beta}\right) \chi_{1} \bar{\chi}_{1} \chi_{2}-\left(\frac{4 \widehat{\eta}^{2}}{\omega_{2}\left(\omega_{2}-2 \omega_{1}\right)}+\frac{6 \widehat{\eta}^{2}}{4 \omega_{1}^{2}-\omega_{2}^{2}}+\widehat{\beta}\right) \chi_{1}^{2} \bar{\chi}_{2} e^{-2 i \widehat{\sigma}_{1} T_{1}} \\
& +\frac{1}{4} \widehat{\delta}_{1} \bar{\chi}_{2} e^{i\left(2 \widehat{\sigma}-2 \widehat{\sigma}_{1}\right) T_{1}} \\
& +\frac{i}{4} \widehat{\delta}_{1} \bar{\chi}_{1} e^{i\left(2 \widehat{\sigma}-2 \widehat{\sigma}_{1}\right) T_{1}}+\frac{3}{4} \widehat{\delta}_{2} \chi_{2} \bar{\chi}_{2}^{2} e^{i\left(2 \widehat{\sigma}-2 \widehat{\sigma}_{1}\right) T_{1}}+\frac{1}{4} \widehat{\delta}_{2} \chi_{2}^{3} e^{i\left(2 \widehat{\sigma}_{1}-2 \widehat{\sigma}\right) T_{1}}+\frac{3 i \widehat{\delta}_{2}}{8} \chi_{1} \bar{\chi}_{2}^{2} e^{i\left(2 \widehat{\sigma}-3 \widehat{\sigma}_{1}\right) T_{1}} \\
& -\frac{3 i}{8} \widehat{\delta}_{2} \chi_{1} \chi_{2}^{2} e^{i\left(\widehat{\sigma}_{1}-2 \widehat{\sigma}\right) T_{1}}+\frac{3 i}{4} \widehat{\delta}_{2} \bar{\chi}_{1} \chi_{2} \bar{\chi}_{2} e^{i\left(2 \widehat{\sigma}-\widehat{\sigma}_{1}\right) T_{1}} \\
& +\frac{3 i}{8} \widehat{\delta}_{2} \chi_{1} \bar{\chi}_{1}^{2} e^{i\left(2 \widehat{\sigma}-\widehat{\sigma}_{1}\right) T_{1}}-\frac{i}{8} \widehat{\delta}_{2} \chi_{1}^{3} e^{i\left(-2 \widehat{\sigma}-\widehat{\sigma}_{1}\right) T_{1}}+\frac{3}{16} \widehat{\delta}_{2} \bar{\chi}_{1}^{2} \bar{\chi}_{2} e^{i\left(4 \widehat{\sigma}-2 \widehat{\sigma}_{1}\right) T_{1}}-\frac{1}{16} \widehat{\delta}_{2} \bar{\chi}_{2}^{3} e^{i\left(4 \widehat{\sigma}-4 \widehat{\sigma}_{1}\right) T_{1}} \\
& -\frac{3 i}{16} \widehat{\delta}_{2} \bar{\chi}_{1} \bar{\chi}_{2}^{2} e^{i\left(4 \widehat{\sigma}-3 \widehat{\sigma}_{1}\right) T_{1}}+\frac{i}{16} \widehat{\delta}_{2} \bar{\chi}_{1}^{3} e^{i\left(4 \widehat{\sigma}-\widehat{\sigma}_{1}\right) T_{1}} .
\end{aligned}
$$


Utilizing the multiple scales reconstitution method [32], we can combine the solvability conditions of equations (28), (30a), and (30b) as

$$
\begin{aligned}
& 2 i \omega_{1} \frac{\mathrm{d}}{\mathrm{d} t} \chi_{1}=\frac{\varepsilon \widehat{f} \Omega^{2}}{2} e^{i\left(\widehat{\sigma} T_{1}+\gamma\right)}-\frac{\varepsilon^{2} \widehat{\sigma} \hat{f} \Omega^{2}}{4 \omega_{1}} e^{i\left(\widehat{\sigma} T_{1}+\gamma\right)}-\varepsilon^{2}\left(i \omega_{1} \widehat{\mu}_{1}+\widehat{\alpha}_{1}+i \omega_{1} \widehat{\alpha}_{2}\right) \chi_{1} \\
& +\varepsilon^{2}\left(\frac{4 \widehat{\eta}^{2}}{\omega_{2}^{2}}-\frac{2 \widehat{\eta}^{2}}{4 \omega_{1}^{2}-\omega_{2}^{2}}-3 \widehat{\beta}-3 \widehat{\alpha}_{3}-i \omega_{1} \widehat{\alpha}_{4}-\omega_{1}^{2} \widehat{\alpha}_{5}\right) \chi_{1}^{2} \bar{\chi}_{1}+\varepsilon^{2}\left(\frac{12 \widehat{\eta}^{2}}{\omega_{2}^{2}}-\frac{4 \widehat{\eta}^{2}}{\omega_{2}\left(\omega_{2}-2 \omega_{1}\right)}-\frac{4 \widehat{\eta}^{2}}{\omega_{2}\left(2 \omega_{1}+\omega_{2}\right)}-2 \widehat{\beta}\right) \chi_{1} \chi_{2} \bar{\chi}_{2} \\
& -\varepsilon^{2}\left(\frac{2 \widehat{\eta}^{2}}{\omega_{2}^{2}}+\frac{4 \widehat{\eta}^{2}}{\omega_{2}\left(\omega_{2}-2 \omega_{1}\right)}+\widehat{\beta}\right) \bar{\chi}_{1} \chi_{2}^{2} e^{2 \widehat{\sigma}_{1} T_{1}}-\frac{1}{4} \varepsilon^{2} \widehat{\delta}_{1} \bar{\chi}_{1} e^{2 \hat{i \sigma} T_{1}}+\frac{i}{4} \varepsilon^{2} \widehat{\delta}_{1} \bar{\chi}_{2} e^{i\left(2 \widehat{\sigma}-\widehat{\sigma}_{1}\right) T_{1}} \\
& -\frac{3}{4} \mathcal{E}^{2} \widehat{\delta}_{2} \chi_{1} \bar{\chi}_{1}^{2} e^{2 \hat{\sigma} T_{1}}-\frac{1}{4} \mathcal{E}^{2} \widehat{\delta}_{2} \chi_{1}^{3} e^{-2 \hat{\imath} T_{1}} \\
& +\frac{3 i}{8} \varepsilon^{2} \widehat{\delta}_{2} \bar{\chi}_{1}^{2} \chi_{2} e^{i\left(2 \widehat{\sigma}+\widehat{\sigma}_{1}\right) T_{1}}-\frac{3 i}{8} \varepsilon^{2} \widehat{\delta}_{2} \chi_{1}^{2} \chi_{2} e^{i\left(-2 \widehat{\sigma}+\widehat{\sigma}_{1}\right) T_{1}}+\frac{3 i}{4} \varepsilon^{2} \widehat{\delta}_{2} \chi_{1} \bar{\chi}_{1} \bar{\chi}_{2} e^{i\left(2 \widehat{\sigma}-\widehat{\sigma}_{1}\right) T_{1}}+\frac{3 i}{8} \varepsilon^{2} \widehat{\delta}_{2} \chi_{2} \bar{\chi}_{2}^{2} e^{i\left(2 \widehat{\sigma}-\widehat{\sigma}_{1}\right) T_{1}} \\
& -\frac{i}{8} \varepsilon^{2} \widehat{\delta}_{2} \chi_{2}^{3} e^{i\left(-2 \widehat{\sigma}+3 \widehat{\sigma}_{1}\right) T_{1}}+\frac{3}{16} \varepsilon^{2} \widehat{\delta}_{2} \bar{\chi}_{1} \bar{\chi}_{2}^{2} e^{i\left(4 \widehat{\sigma}-2 \widehat{\sigma}_{1}\right) T_{1}}-\frac{1}{16} \varepsilon^{2} \widehat{\delta}_{2} \bar{\chi}_{1}^{3} e^{4 \hat{\sigma} T_{1}}-\frac{i}{16} \varepsilon^{2} \widehat{\delta}_{2} \bar{\chi}_{2}^{3} e^{i\left(4 \widehat{\sigma}-3 \widehat{\sigma}_{1}\right) T_{1}}+\frac{3 i}{16} \varepsilon^{2} \widehat{\delta}_{2} \bar{\chi}_{1}^{2} \bar{\chi}_{2} e^{i\left(4 \widehat{\sigma}^{-}-\widehat{\sigma}_{1}\right) T_{1}}, \\
& 2 i \omega_{2} \frac{\mathrm{d}}{\mathrm{d} t} \chi_{2}=-\frac{i \varepsilon \widehat{f} \Omega^{2}}{2} e^{i\left(\left(\widehat{\sigma}-\widehat{\sigma}_{1}\right) T_{1}+\gamma\right)}-\frac{i \varepsilon^{2}\left(\widehat{\sigma}_{1}-\widehat{\sigma}\right) \widehat{f} \Omega^{2}}{4 \omega_{2}} e^{i\left(\left(\widehat{\sigma}-\widehat{\sigma}_{1}\right) T_{1}+\gamma\right)}-\varepsilon^{2}\left(i \omega_{2} \widehat{\mu}_{2}+\widehat{\alpha}_{1}+i \omega_{2} \widehat{\alpha}_{2}\right) \chi_{2} \\
& +\varepsilon^{2}\left(\frac{30 \widehat{\eta}^{2}}{\omega_{2}^{2}}-3 \widehat{\beta}-3 \widehat{\alpha}_{3}-i \omega_{2} \widehat{\alpha}_{4}-\omega_{2}^{2} \widehat{\alpha}_{5}\right) \chi_{2}^{2} \bar{A}_{2}-\varepsilon^{2}\left(\frac{4 \widehat{\eta}^{2}}{\omega_{2}\left(2 \omega_{1}+\omega_{2}\right)}+\frac{4 \widehat{\eta}^{2}}{\omega_{2}\left(\omega_{2}-2 \omega_{1}\right)}-\frac{12 \widehat{\eta}^{2}}{\omega_{2}^{2}}+2 \widehat{\beta}\right) \chi_{1} \bar{\chi}_{1} \chi_{2} \\
& -\varepsilon^{2}\left(\frac{4 \widehat{\eta}^{2}}{\omega_{2}\left(\omega_{2}-2 \omega_{1}\right)}+\frac{6 \widehat{\eta}^{2}}{4 \omega_{1}^{2}-\omega_{2}^{2}}+\widehat{\beta}\right) \chi_{1}^{2} \bar{\chi}_{2} e^{-2 \widehat{\sigma}_{1} T_{1}}+\frac{1}{4} \varepsilon^{2} \widehat{\delta}_{1} \bar{\chi}_{2} e^{i\left(2 \widehat{\sigma}-2 \widehat{\sigma}_{1}\right) T_{1}} \\
& +\frac{i}{4} \varepsilon^{2} \widehat{\delta}_{1} \bar{\chi}_{1} e^{i\left(2 \widehat{\sigma}-\widehat{\sigma}_{1}\right) T_{1}}+\frac{3}{4} \varepsilon^{2} \widehat{\delta}_{2} \chi_{2} \bar{\chi}_{2}^{2} e^{i\left(2 \widehat{\sigma}-2 \widehat{\sigma}_{1}\right) T_{1}} \\
& +\frac{1}{4} \varepsilon^{2} \widehat{\delta}_{2} \chi_{2}^{3} e^{i\left(2 \widehat{\sigma}_{1}-2 \widehat{\sigma}\right) T_{1}}+\frac{3 i}{8} \varepsilon^{2} \widehat{\delta}_{2} \chi_{1} \bar{\chi}_{2}^{2} e^{i\left(2 \widehat{\sigma}-3 \widehat{\sigma}_{1}\right) T_{1}}-\frac{3 i}{8} \varepsilon^{2} \widehat{\delta}_{2} \chi_{1} \chi_{2}^{2} e^{i\left(\widehat{\sigma}_{1}-2 \widehat{\sigma}\right) T_{1}} \\
& +\frac{3 i}{4} \varepsilon^{2} \widehat{\delta}_{2} \bar{\chi}_{1} \chi_{2} \bar{\chi}_{2} e^{i\left(2 \widehat{\sigma}-\widehat{\sigma}_{1}\right) T_{1}}+\frac{3 i}{8} \varepsilon^{2} \widehat{\delta}_{2} \chi_{1} \bar{\chi}_{1}^{2} e^{i\left(2 \widehat{\sigma}-\widehat{\sigma}_{1}\right) T_{1}} \\
& -\frac{i}{8} \varepsilon^{2} \widehat{\delta}_{2} \chi_{1}^{3} e^{i\left(-2 \widehat{\sigma}-\widehat{\sigma}_{1}\right) T_{1}}+\frac{3}{16} \varepsilon^{2} \widehat{\delta}_{2} \bar{\chi}_{1}^{2} \bar{\chi}_{2} e^{i\left(4 \widehat{\sigma}-2 \widehat{\sigma}_{1}\right) T_{1}}-\frac{1}{16} \varepsilon^{2} \widehat{\delta}_{2} \bar{\chi}_{2}^{3} e^{i\left(\widehat{4}-\widehat{\sigma_{1}}\right) T_{1}} \\
& -\frac{3 i}{16} \varepsilon^{2} \widehat{\delta}_{2} \bar{\chi}_{1} \bar{\chi}_{2}^{2} e^{i\left(4 \widehat{\sigma}-3 \widehat{\sigma}_{1}\right) T_{1}}+\frac{i}{16} \varepsilon^{2} \widehat{\delta}_{2} \bar{\chi}_{1}^{3} e^{i\left(4 \widehat{\sigma}-\widehat{\sigma}_{1}\right) T_{1}} \text {. }
\end{aligned}
$$

To analyze equations (31a) and (31b), the functions $\chi_{1}\left(T_{1}, T_{2}\right)$ and $\chi_{2}\left(T_{1}, T_{2}\right)$ can be expressed in polar form as follows:

$\chi_{j}=\frac{1}{2} a_{j}(\tau) e^{i \theta_{j}(\tau)} \Rightarrow \frac{\mathrm{d}}{\mathrm{d} t} \chi_{j}=\frac{1}{2} \dot{a}_{j}(\tau) e^{i \theta_{j}(\tau)}+\frac{i}{2} a_{j}(\tau) \dot{\theta}_{j}(\tau) e^{i \theta_{j}(\tau)}, \quad j=1,2$, where $a_{1}$ and $a_{2}$ denote the rotating disk vibration amplitudes in $V_{1}$ and $V_{2}$ directions, respectively, and $\theta_{1}$ and $\theta_{2}$ are the motion phase angles. Substituting equation (32) into equations (31a) and (31b), one can derive the following amplitude-phase modulation equations: 


$$
\begin{aligned}
\frac{\mathrm{d} a_{1}}{\mathrm{~d} \tau}= & \left(1-\frac{\sigma}{2 \omega_{1}}\right) \frac{f \Omega^{2}}{2 \omega_{1}} \sin \left(\phi_{1}+\gamma\right)-\frac{1}{2}\left(\mu_{1}+\alpha_{2}\right) a_{1}-\frac{1}{8} \alpha_{4} a_{1}^{3}-\frac{1}{8 \omega_{1}}\left(\frac{2 \eta^{2}}{\omega_{2}^{2}}+\frac{4 \eta^{2}}{\omega_{2}\left(\omega_{2}-2 \omega_{1}\right)}+\beta\right) a_{1} a_{2}^{2} \sin \left(2 \phi_{1}-2 \phi_{2}\right) \\
& -\frac{1}{8 \omega_{1}} \delta_{1} a_{1} \sin \left(2 \phi_{1}\right)+\frac{1}{8 \omega_{1}} \delta_{1} a_{2} \cos \left(\phi_{1}+\phi_{2}\right)-\frac{3}{32 \omega_{1}} \delta_{2} a_{1}^{3} \sin \left(2 \phi_{1}\right)+\frac{1}{32 \omega_{1}} \delta_{2} a_{1}^{3} \sin \left(2 \phi_{1}\right)+\frac{3}{64 \omega_{1}} \delta_{2} a_{1}^{2} a_{2} \cos \left(3 \phi_{1}-\phi_{2}\right) \\
& -\frac{3}{64 \omega_{1}} \delta_{2} a_{1}^{2} a_{2} \cos \left(\phi_{1}+\phi_{2}\right)+\frac{3}{32 \omega_{1}} \delta_{2} a_{1}^{2} a_{2} \cos \left(\phi_{1}+\phi_{2}\right)+\frac{3}{64 \omega_{1}} \delta_{2} a_{2}^{3} \cos \left(\phi_{1}+\phi_{2}\right)-\frac{1}{64 \omega_{1}} \delta_{2} a_{2}^{3} \cos \left(\phi_{1}-3 \phi_{2}\right) \\
& +\frac{3}{128 \omega_{1}} \delta_{2} a_{1} a_{2}^{2} \sin \left(2 \phi_{1}+2 \phi_{2}\right)-\frac{1}{128 \omega_{1}} \delta_{2} a_{1}^{3} \sin \left(4 \phi_{1}\right)-\frac{1}{128 \omega_{1}} \delta_{2} a_{2}^{3} \cos \left(\phi_{1}+3 \phi_{2}\right)+\frac{3}{128 \omega_{1}} \delta_{2} a_{1}^{2} a_{2} \cos \left(3 \phi_{1}+\phi_{2}\right)
\end{aligned}
$$

$$
\begin{aligned}
\frac{\mathrm{d} a_{2}}{\mathrm{~d} \tau}= & \left(\frac{\sigma}{2 \omega_{2}}-\frac{\sigma_{1}}{2 \omega_{2}}-1\right) \frac{f \Omega^{2}}{2 \omega_{2}} \cos \left(\phi_{2}+\gamma\right)-\frac{1}{2}\left(\mu_{2}+\alpha_{2}\right) a_{2}-\frac{1}{8} \alpha_{4} a_{2}^{3}-\frac{1}{8 \omega_{2}}\left(\frac{4 \eta^{2}}{\omega_{2}\left(\omega_{2}-2 \omega_{1}\right)}+\frac{6 \eta^{2}}{4 \omega_{1}^{2}-\omega_{2}^{2}}+\beta\right) a_{1}^{2} a_{2} \sin \left(-2 \phi_{1}+2 \phi_{2}\right) \\
& +\frac{1}{8 \omega_{2}} \delta_{1} a_{2} \sin \left(2 \phi_{2}\right)+\frac{1}{8 \omega_{2}} \delta_{1} a_{1} \cos \left(\phi_{1}+\phi_{2}\right)+\frac{3}{32 \omega_{2}} \delta_{2} a_{2}^{3} \sin \left(2 \phi_{2}\right)-\frac{1}{32 \omega_{2}} \delta_{2} a_{2}^{3} \sin \left(2 \phi_{2}\right)+\frac{3}{64 \omega_{2}} \delta_{2} a_{1} a_{2}^{2} \cos \left(3 \phi_{2}-\phi_{1}\right) \\
& -\frac{3}{64 \omega_{2}} \delta_{2} a_{1} a_{2}^{2} \cos \left(\phi_{1}+\phi_{2}\right)+\frac{3}{32 \omega_{2}} \delta_{2} a_{1} a_{2}^{2} \cos \left(\phi_{1}+\phi_{2}\right)+\frac{3}{64 \omega_{2}} \delta_{2} a_{1}^{3} \cos \left(\phi_{1}+\phi_{2}\right)-\frac{1}{64 \omega_{2}} \delta_{2} a_{1}^{3} \cos \left(\phi_{2}-3 \phi_{1}\right) \\
& +\frac{3}{128 \omega_{2}} \delta_{2} a_{1}^{2} a_{2} \sin \left(2 \phi_{1}+2 \phi_{2}\right)-\frac{1}{128 \omega_{2}} \delta_{2} a_{2}^{3} \sin \left(4 \phi_{2}\right)-\frac{3}{128 \omega_{2}} \delta_{2} a_{1} a_{2}^{2} \cos \left(\phi_{1}+3 \phi_{2}\right)+\frac{1}{128 \omega_{2}} \delta_{2} a_{1}^{3} \cos \left(3 \phi_{1}+\phi_{2}\right),
\end{aligned}
$$

$$
\begin{aligned}
& \frac{\mathrm{d} \phi_{1}}{\mathrm{~d} \tau}=\sigma+\left(1-\frac{\sigma}{2 \omega_{1}}\right) \frac{f \Omega^{2}}{2 \omega_{1} a_{1}} \cos \left(\phi_{1}+\gamma\right)-\frac{\alpha_{1}}{2 \omega_{1}}+\frac{1}{8 \omega_{1}}\left(\frac{4 \eta^{2}}{\omega_{2}^{2}}-\frac{2 \eta^{2}}{4 \omega_{1}^{2}-\omega_{2}^{2}}-3 \beta-3 \alpha_{3}-\omega_{1}^{2} \alpha_{5}\right) a_{1}^{2} \\
& +\frac{1}{8 \omega_{1}}\left(\frac{12 \eta^{2}}{\omega_{2}^{2}}-\frac{4 \eta^{2}}{\omega_{2}\left(\omega_{2}-2 \omega_{1}\right)}-\frac{4 \eta^{2}}{\omega_{2}\left(2 \omega_{1}+\omega_{2}\right)}-2 \beta\right) a_{2}^{2}-\frac{1}{8 \omega_{1}}\left(\frac{2 \eta^{2}}{\omega_{2}^{2}}+\frac{4 \eta^{2}}{\omega_{2}\left(\omega_{2}-2 \omega_{1}\right)}+\beta\right) a_{2}^{2} \cos \left(2 \phi_{1}-2 \phi_{2}\right) \\
& -\frac{1}{8 \omega_{1}} \delta_{1} \cos \left(2 \phi_{1}\right)-\frac{1}{8 \omega_{1} a_{1}} \delta_{1} a_{2} \sin \left(\phi_{1}+\phi_{2}\right)-\frac{3}{32 \omega_{1}} \delta_{2} a_{1}^{2} \cos \left(2 \phi_{1}\right)-\frac{1}{32 \omega_{1}} \delta_{2} a_{1}^{2} \cos \left(2 \phi_{1}\right) \\
& -\frac{3}{64 \omega_{1}} \delta_{2} a_{1} a_{2} \sin \left(3 \phi_{1}-\phi_{2}\right)-\frac{3}{64 \omega_{1}} \delta_{2} a_{1} a_{2} \sin \left(\phi_{1}+\phi_{2}\right)-\frac{3}{32 \omega_{1}} \delta_{2} a_{1} a_{2} \sin \left(\phi_{1}+\phi_{2}\right) \\
& -\frac{3}{64 \omega_{1} a_{1}} \delta_{2} a_{2}^{3} \sin \left(\phi_{1}+\phi_{2}\right)+\frac{1}{64 \omega_{1} a_{1}} \delta_{2} a_{2}^{3} \sin \left(\phi_{1}-3 \phi_{2}\right)+\frac{3}{128 \omega_{1}} \delta_{2} a_{2}^{2} \cos \left(2 \phi_{1}+2 \phi_{2}\right) \\
& -\frac{1}{128 \omega_{1}} \delta_{2} a_{1}^{2} \cos \left(4 \phi_{1}\right)+\frac{1}{128 \omega_{1} a_{1}} \delta_{2} a_{2}^{3} \sin \left(\phi_{1}+3 \phi_{2}\right)-\frac{3}{128 \omega_{1}} \delta_{2} a_{1} a_{2} \sin \left(3 \phi_{1}+\phi_{2}\right), \\
& \frac{\mathrm{d} \phi_{2}}{\mathrm{~d} \tau}=\sigma-\sigma_{1}-\left(\frac{\sigma}{2 \omega_{2}}-\frac{\sigma_{1}}{2 \omega_{2}}-1\right) \frac{f \Omega^{2}}{2 \omega_{2} a_{2}} \sin \left(\phi_{2}+\gamma\right)-\frac{\alpha_{1}}{2 \omega_{2}}+\frac{1}{8 \omega_{2}}\left(\frac{30 \eta^{2}}{\omega_{2}^{2}}-3 \beta-3 \alpha_{3}-\omega_{2}^{2} \alpha_{5}\right) a_{2}^{2} \\
& -\frac{1}{8 \omega_{2}}\left(\frac{4 \eta^{2}}{\omega_{2}\left(2 \omega_{1}+\omega_{2}\right)}+\frac{4 \eta^{2}}{\omega_{2}\left(\omega_{2}-2 \omega_{1}\right)}-\frac{12 \eta^{2}}{\omega_{2}^{2}}+2 \beta\right) a_{1}^{2}-\frac{1}{8 \omega_{2}}\left(\frac{4 \eta^{2}}{\omega_{2}\left(\omega_{2}-2 \omega_{1}\right)}+\frac{6 \eta^{2}}{4 \omega_{1}^{2}-\omega_{2}^{2}}+\beta\right) a_{1}^{2} \cos \left(-2 \phi_{1}+2 \phi_{2}\right) \\
& +\frac{1}{8 \omega_{2}} \delta_{1} \cos \left(2 \phi_{2}\right)-\frac{1}{8 \omega_{2} a_{2}} \delta_{1} a_{1} \sin \left(\phi_{1}+\phi_{2}\right)+\frac{3}{32 \omega_{2}} \delta_{2} a_{2}^{2} \cos \left(2 \phi_{2}\right)+\frac{1}{32 \omega_{2}} \delta_{2} a_{2}^{2} \cos \left(2 \phi_{2}\right)-\frac{3}{64 \omega_{2}} \delta_{2} a_{1} a_{2} \sin \left(3 \phi_{2}-\phi_{1}\right) \\
& -\frac{3}{64 \omega_{2}} \delta_{2} a_{1} a_{2} \sin \left(\phi_{1}+\phi_{2}\right)-\frac{3}{32 \omega_{2}} \delta_{2} a_{1} a_{2} \sin \left(\phi_{1}+\phi_{2}\right)-\frac{3}{64 \omega_{2} a_{2}} \delta_{2} a_{1}^{3} \sin \left(\phi_{1}+\phi_{2}\right)+\frac{1}{64 \omega_{2} a_{2}} \delta_{2} a_{1}^{3} \sin \left(\phi_{2}-3 \phi_{1}\right) \\
& +\frac{3}{128 \omega_{2}} \delta_{2} a_{1}^{2} \cos \left(2 \phi_{1}+2 \phi_{2}\right)-\frac{1}{128 \omega_{2}} \delta_{2} a_{2} \cos \left(4 \phi_{2}\right)+\frac{3}{128 \omega_{2}} \delta_{2} a_{1} a_{2} \sin \left(\phi_{1}+3 \phi_{2}\right)-\frac{1}{128 \omega_{2} a_{2}} \delta_{2} a_{1}^{3} \sin \left(3 \phi_{2}-\phi_{1}\right),
\end{aligned}
$$

where $\phi_{1}=\sigma \tau-\theta_{1}$ and $\phi_{2}=\left(\sigma-\sigma_{1}\right) \tau-\theta_{2}$. Now, by inserting equation (32) into equations (24a) and (24b) and then inserting the obtained equations into equations (18a) and (18b), one can derive a periodic solution for the system equations of motion (i.e., equations (17a) and (17b) when $\left.\delta_{3}=0.0\right)$ as follows: 


$$
\begin{aligned}
& z_{1}(\tau)=a_{1}(\tau) \cos \left(\Omega \tau-\phi_{1}(\tau)\right), \\
& z_{2}(\tau)=a_{2}(\tau) \cos \left(\Omega \tau-\phi_{2}(\tau)\right) .
\end{aligned}
$$

The oscillation amplitudes $\left(a_{1}(\tau) \& a_{2}(\tau)\right)$ and the phase angles $\left(\phi_{1}(\tau) \& \phi_{2}(\tau)\right)$ are governed by the autonomous dynamical system given by equations (33a)-(33d). Setting $\left(\mathrm{d} a_{1} / \mathrm{d} \tau\right)=\left(\mathrm{d} a_{2} / \mathrm{d} \tau\right)=\left(\mathrm{d} \phi_{1} / \mathrm{d} \tau\right)=\left(\mathrm{d} \phi_{2} / \mathrm{d} \tau\right)=0$ in equations (33a)-(33d), we have

$$
\begin{aligned}
& \left(1-\frac{\sigma}{2 \omega_{1}}\right) \frac{f \Omega^{2}}{2 \omega_{1}} \sin \left(\phi_{1}+\gamma\right)-\frac{1}{2}\left(\mu_{1}+\alpha_{2}\right) a_{1}-\frac{1}{8} \alpha_{4} a_{1}^{3}-\frac{1}{8 \omega_{1}}\left(\frac{2 \eta^{2}}{\omega_{2}^{2}}+\frac{4 \eta^{2}}{\omega_{2}\left(\omega_{2}-2 \omega_{1}\right)}+\beta\right) \times a_{1} a_{2}^{2} \sin \left(2 \phi_{1}-2 \phi_{2}\right) \\
& -\frac{1}{8 \omega_{1}} \delta_{1} a_{1} \sin \left(2 \phi_{1}\right)+\frac{1}{8 \omega_{1}} \delta_{1} a_{2} \cos \left(\phi_{1}+\phi_{2}\right)-\frac{3}{32 \omega_{1}} \delta_{2} a_{1}^{3} \sin \left(2 \phi_{1}\right)+\frac{1}{32 \omega_{1}} \delta_{2} a_{1}^{3} \sin \left(2 \phi_{1}\right)+\frac{3}{64 \omega_{1}} \delta_{2} a_{1}^{2} a_{2} \cos \left(3 \phi_{1}-\phi_{2}\right) \\
& -\frac{3}{64 \omega_{1}} \delta_{2} a_{1}^{2} a_{2} \cos \left(\phi_{1}+\phi_{2}\right)+\frac{3}{32 \omega_{1}} \delta_{2} a_{1}^{2} a_{2} \cos \left(\phi_{1}+\phi_{2}\right)+\frac{3}{64 \omega_{1}} \delta_{2} a_{2}^{3} \cos \left(\phi_{1}+\phi_{2}\right)-\frac{1}{64 \omega_{1}} \delta_{2} a_{2}^{3} \cos \left(\phi_{1}-3 \phi_{2}\right) \\
& +\frac{3}{128 \omega_{1}} \delta_{2} a_{1} a_{2}^{2} \sin \left(2 \phi_{1}+2 \phi_{2}\right)-\frac{1}{128 \omega_{1}} \delta_{2} a_{1}^{3} \sin \left(4 \phi_{1}\right)-\frac{1}{128 \omega_{1}} \delta_{2} a_{2}^{3} \cos \left(\phi_{1}+3 \phi_{2}\right)+\frac{3}{128 \omega_{1}} \delta_{2} a_{1}^{2} a_{2} \cos \left(3 \phi_{1}+\phi_{2}\right)=0 \\
& \left(\frac{\sigma}{2 \omega_{2}}-\frac{\sigma_{1}}{2 \omega_{2}}-1\right) \frac{f \Omega^{2}}{2 \omega_{2}} \cos \left(\phi_{2}+\gamma\right)-\frac{1}{2}\left(\mu_{2}+\alpha_{2}\right) a_{2}-\frac{1}{8} \alpha_{4} a_{2}^{3}-\frac{1}{8 \omega_{2}}\left(\frac{4 \eta^{2}}{\omega_{2}\left(\omega_{2}-2 \omega_{1}\right)}+\frac{6 \eta^{2}}{4 \omega_{1}^{2}-\omega_{2}^{2}}+\beta\right) \times a_{1}^{2} a_{2} \sin \left(-2 \phi_{1}+2 \phi_{2}\right) \\
& +\frac{1}{8 \omega_{2}} \delta_{1} a_{2} \sin \left(2 \phi_{2}\right)+\frac{1}{8 \omega_{2}} \delta_{1} a_{1} \cos \left(\phi_{1}+\phi_{2}\right)+\frac{3}{32 \omega_{2}} \delta_{2} a_{2}^{3} \sin \left(2 \phi_{2}\right)-\frac{1}{32 \omega_{2}} \delta_{2} a_{2}^{3} \sin \left(2 \phi_{2}\right) \\
& +\frac{3}{64 \omega_{2}} \delta_{2} a_{1} a_{2}^{2} \cos \left(3 \phi_{2}-\phi_{1}\right)-\frac{3}{64 \omega_{2}} \delta_{2} a_{1} a_{2}^{2} \cos \left(\phi_{1}+\phi_{2}\right)+\frac{3}{32 \omega_{2}} \delta_{2} a_{1} a_{2}^{2} \cos \left(\phi_{1}+\phi_{2}\right)+\frac{3}{64 \omega_{2}} \delta_{2} a_{1}^{3} \cos \left(\phi_{1}+\phi_{2}\right) \\
& -\frac{1}{64 \omega_{2}} \delta_{2} a_{1}^{3} \cos \left(\phi_{2}-3 \phi_{1}\right) \\
& +\frac{3}{128 \omega_{2}} \delta_{2} a_{1}^{2} a_{2} \sin \left(2 \phi_{1}+2 \phi_{2}\right)-\frac{1}{128 \omega_{2}} \delta_{2} a_{2}^{3} \sin \left(4 \phi_{2}\right)-\frac{3}{128 \omega_{2}} \delta_{2} a_{1} a_{2}^{2} \cos \left(\phi_{1}+3 \phi_{2}\right)+\frac{1}{128 \omega_{2}} \delta_{2} a_{1}^{3} \cos \left(3 \phi_{1}+\phi_{2}\right)=0 \text {, } \\
& \sigma+\left(1-\frac{\sigma}{2 \omega_{1}}\right) \frac{f \Omega^{2}}{2 \omega_{1} a_{1}} \cos \left(\phi_{1}+\gamma\right)-\frac{\alpha_{1}}{2 \omega_{1}}+\frac{1}{8 \omega_{1}}\left(\frac{4 \eta^{2}}{\omega_{2}^{2}}-\frac{2 \eta^{2}}{4 \omega_{1}^{2}-\omega_{2}^{2}}-3 \beta-3 \alpha_{3}-\omega_{1}^{2} \alpha_{5}\right) a_{1}^{2} \\
& +\frac{1}{8 \omega_{1}}\left(\frac{12 \eta^{2}}{\omega_{2}^{2}}-\frac{4 \eta^{2}}{\omega_{2}\left(\omega_{2}-2 \omega_{1}\right)}-\frac{4 \eta^{2}}{\omega_{2}\left(2 \omega_{1}+\omega_{2}\right)}-2 \beta\right) a_{2}^{2} \\
& -\frac{1}{8 \omega_{1}}\left(\frac{2 \eta^{2}}{\omega_{2}^{2}}+\frac{4 \eta^{2}}{\omega_{2}\left(\omega_{2}-2 \omega_{1}\right)}+\beta\right) a_{2}^{2} \cos \left(2 \phi_{1}-2 \phi_{2}\right)-\frac{1}{8 \omega_{1}} \delta_{1} \cos \left(2 \phi_{1}\right)-\frac{1}{8 \omega_{1} a_{1}} \delta_{1} a_{2} \sin \left(\phi_{1}+\phi_{2}\right)-\frac{3}{32 \omega_{1}} \delta_{2} a_{1}^{2} \cos \left(2 \phi_{1}\right) \\
& -\frac{1}{32 \omega_{1}} \delta_{2} a_{1}^{2} \cos \left(2 \phi_{1}\right)-\frac{3}{64 \omega_{1}} \delta_{2} a_{1} a_{2} \sin \left(3 \phi_{1}-\phi_{2}\right)-\frac{3}{64 \omega_{1}} \delta_{2} a_{1} a_{2} \sin \left(\phi_{1}+\phi_{2}\right)-\frac{3}{32 \omega_{1}} \delta_{2} a_{1} a_{2} \sin \left(\phi_{1}+\phi_{2}\right) \\
& -\frac{3}{64 \omega_{1} a_{1}} \delta_{2} a_{2}^{3} \sin \left(\phi_{1}+\phi_{2}\right)+\frac{1}{64 \omega_{1} a_{1}} \delta_{2} a_{2}^{3} \sin \left(\phi_{1}-3 \phi_{2}\right) \\
& +\frac{3}{128 \omega_{1}} \delta_{2} a_{2}^{2} \cos \left(2 \phi_{1}+2 \phi_{2}\right)-\frac{1}{128 \omega_{1}} \delta_{2} a_{1}^{2} \cos \left(4 \phi_{1}\right)+\frac{1}{128 \omega_{1} a_{1}} \delta_{2} a_{2}^{3} \sin \left(\phi_{1}+3 \phi_{2}\right)-\frac{3}{128 \omega_{1}} \delta_{2} a_{1} a_{2} \sin \left(3 \phi_{1}+\phi_{2}\right)=0 \text {, }
\end{aligned}
$$




$$
\begin{aligned}
\sigma- & \sigma_{1}-\left(\frac{\sigma}{2 \omega_{2}}-\frac{\sigma_{1}}{2 \omega_{2}}-1\right) \frac{f \Omega^{2}}{2 \omega_{2} a_{2}} \sin \left(\phi_{2}+\gamma\right)-\frac{\alpha_{1}}{2 \omega_{2}}+\frac{1}{8 \omega_{2}}\left(\frac{30 \eta^{2}}{\omega_{2}^{2}}-3 \beta-3 \alpha_{3}-\omega_{2}^{2} \alpha_{5}\right) a_{2}^{2} \\
& -\frac{1}{8 \omega_{2}}\left(\frac{4 \eta^{2}}{\omega_{2}\left(2 \omega_{1}+\omega_{2}\right)}+\frac{4 \eta^{2}}{\omega_{2}\left(\omega_{2}-2 \omega_{1}\right)}-\frac{12 \eta^{2}}{\omega_{2}^{2}}+2 \beta\right) a_{1}^{2}-\frac{1}{8 \omega_{2}}\left(\frac{4 \eta^{2}}{\omega_{2}\left(\omega_{2}-2 \omega_{1}\right)}+\frac{6 \eta^{2}}{4 \omega_{1}^{2}-\omega_{2}^{2}}+\beta\right) a_{1}^{2} \cos \left(-2 \phi_{1}+2 \phi_{2}\right) \\
& +\frac{1}{8 \omega_{2}} \delta_{1} \cos \left(2 \phi_{2}\right)-\frac{1}{8 \omega_{2} a_{2}} \delta_{1} a_{1} \sin \left(\phi_{1}+\phi_{2}\right)+\frac{3}{32 \omega_{2}} \delta_{2} a_{2}^{2} \cos \left(2 \phi_{2}\right)+\frac{1}{32 \omega_{2}} \delta_{2} a_{2}^{2} \cos \left(2 \phi_{2}\right)-\frac{3}{64 \omega_{2}} \delta_{2} a_{1} a_{2} \sin \left(3 \phi_{2}-\phi_{1}\right) \\
& -\frac{3}{64 \omega_{2}} \delta_{2} a_{1} a_{2} \sin \left(\phi_{1}+\phi_{2}\right)-\frac{3}{32 \omega_{2}} \delta_{2} a_{1} a_{2} \sin \left(\phi_{1}+\phi_{2}\right)-\frac{3}{64 \omega_{2} a_{2}} \delta_{2} a_{1}^{3} \sin \left(\phi_{1}+\phi_{2}\right)+\frac{1}{64 \omega_{2} a_{2}} \delta_{2} a_{1}^{3} \sin \left(\phi_{2}-3 \phi_{1}\right) \\
& +\frac{3}{128 \omega_{2}} \delta_{2} a_{1}^{2} \cos \left(2 \phi_{1}+2 \phi_{2}\right)-\frac{1}{128 \omega_{2}} \delta_{2} a_{2} \cos \left(4 \phi_{2}\right)+\frac{3}{128 \omega_{2}} \delta_{2} a_{1} a_{2} \sin \left(\phi_{1}+3 \phi_{2}\right)-\frac{1}{128 \omega_{2} a_{2}} \delta_{2} a_{1}^{3} \sin \left(3 \phi_{1}+\phi_{2}\right)=0
\end{aligned}
$$

The nonlinear system of algebraic equations given by equations (35a)-(35d) governs the steady-state vibration amplitudes $\left(a_{1} \& a_{2}\right)$ of the system given by equations (17a) and (17b) when $\delta_{3}=0.0$. By solving equations (35a)-(35d) numerically in terms of the parameters $\sigma, f, \delta_{1}$, and $\delta_{2}$, one can obtain the different response curves as in Section 4 .

\section{Results and Discussion}

Based on the introduced dimensionless variables that are given in equations (17a) and (17b) (i.e., $z_{1}=\left(v_{1} / h_{0}\right)$, $\left.z_{2}=\left(v_{2} / h_{0}\right)\right)$ and the periodic solution that is given by equations (34a) and (34b), it can be deduced that the actual solution of the physical system (i.e., $v_{1}(t)$ and $\left.v_{2}(t)\right)$ is also periodic because $h_{0}$ is a constant representing the air-gap size. Accordingly, the rotor can oscillate without physical contact with the pole legs if $\left|z_{1}\right|=\left|v_{1} / h_{0}\right|<1$ and $\left|z_{2}\right|=\left|v_{2} / h_{0}\right|<1$. This means that $\left|z_{1}\right|=\mid a_{1}(\tau) \cos$ $\left(\Omega \tau-\phi_{1}(\tau) \mid<1 \quad\right.$ and $\quad\left|z_{2}\right|=\mid a_{2}(\tau) \cos \left(\Omega \tau-\phi_{2}(\tau) \mid<1\right.$ depending on equations (34a) and (34b). Therefore, the sufficient conditions for the oscillation of the rotating disk without any physical contact with the pole leg are that $a_{1}<1$ and $a_{2}<1$. Hence, equations (35a) $-(35 \mathrm{~d})$ govern the steady-state vibration amplitudes in $V_{1}$ and $V_{2}$ as long as $a_{1}<1$ and $a_{2}<1$; otherwise, the impact and rub forces occur that require the investigation equations (17a) and (17b) when $\delta_{3} \neq 0$. Because the system model given by equations (17a) and (17b) is a strong discontinuous dynamical system when $\delta_{3} \neq 0$, it can be studied numerically using the temporal oscillations, frequency spectrum, Poincare map, and bifurcation diagrams when the rub and impact forces occur (i.e., when $a_{1} \geq 1$ and/or $a_{2} \geq 1$ ). Based on this explanation, the oscillatory behaviours of the system model given by equations (17a) and (17b) can be investigated via solving the algebraic equations (i.e., equations (35a)-(35d)) as long as $a_{1}<1$ and $a_{2}<1$. But, when the rub and impact occur (i.e., $a_{1} \geq 1$ and/or $a_{2} \geq 1$ ), the system model is analyzed numerically via solving equations (17a) and (17b). It is worth mentioning that the numerical simulations in the whole article are performed utilizing MATLAB ODE45 solver to obtain the system the bifurcation diagrams, temporal oscillations, orbit plot, and frequency spectrum.
The dynamical characteristics of the considered system are analyzed utilizing the dimensionless system parameters [26-28]: $\mu_{1}=0.015, \mu_{2}=0.025, \Omega=\omega_{1}+\sigma, f=0.025, \lambda=$ $1, \rho=0.05, \eta=\lambda \rho, \quad \delta_{1}=0.1, \delta_{2}=0.1, \delta_{3}=5.0, \quad \omega_{1}=\sqrt{1+}$ $\lambda^{2} \rho+(1 / 2) \delta_{1}, \omega_{2}=\sqrt{1+3 \lambda^{2} \rho+(1 / 2) \delta_{1}}, \quad \beta=\rho+(3 / 8)$ $\delta_{2}, p=1, d=0.025, \alpha_{1}=p-1, \quad \alpha_{2}=d, \alpha_{3}=-2+3 p-$ $p^{2}, \alpha_{4}=3 d-2 p d, \alpha_{5}=-d^{2}$. The rest of Section 4 is organized in a way such that Section 4.1 is assigned to explore the effects of both the linear asymmetric stiffness coefficient $\delta_{1}$ and the nonlinear asymmetric stiffness coefficient $\delta_{2}$ on dynamical behaviours of the rotating disk when the controller is turned off, while the effects of the different control parameters on the system dynamics are explored in Section 4.2 .

4.1. Rub and Impact Forces of the System without Control. The effects of the stiffness asymmetry on both the lateral vibrations and the rub-impact occurrence are discussed when the controller is turned off (i.e., $\alpha_{i}=0, i=1,2, \ldots, 5$ ). Referring to equation (26), the parameter $\sigma$ describes the closeness of the rotor speed $\Omega$ to its natural frequency $\omega_{1}$, while $\sigma_{1}$ is constant such that $\sigma_{1}=\omega_{2}-$ $\omega_{1}=\sqrt{1+3 \lambda^{2} \rho+(1 / 2) \delta_{1}}-\sqrt{1+\lambda^{2} \rho+(1 / 2) \delta_{1}}$. Therefore, $\sigma$ is utilized as a bifurcation control parameter in the whole article to investigate the system dynamics when $\Omega$ is close to $\omega_{1}$ (i.e., studying the primary resonance $\Omega \longrightarrow \omega_{1}$ ). Accordingly, the amplitude of oscillations (i.e., $a_{1}$ and $a_{2}$ ) in $V_{1}$ and $V_{2}$ directions at resonance condition (i.e., $\sigma \cong 0$ ) can be predicted via solving equations (35a)-(35d) numerically utilizing $\sigma$ as a bifurcation control parameter (see Figures 2 and 3). In addition, the occurrence of the rub and impact forces between the rotor and stator can be predicted if $a_{1} \geq 1$ and/or $a_{2} \geq 1$ as explained above.

In Figure 2, the system oscillation amplitudes $a_{1}$ and $a_{2}$ are plotted versus $\sigma$ via solving equations (35a)-(35d) numerically when $\delta_{1}=0.05$ and $\delta_{2}=0.0$, while Figure 3 shows the system lateral vibration amplitudes $a_{1}$ and $a_{2}$ when $\delta_{1}=$ 0.1 and $\delta_{2}=0.0$. It is clear from Figures 2 and 3 that the increase of $\delta_{1}$ from $\delta_{1}=0.05$ to $\delta_{1}=0.1$ increases the system oscillation amplitudes and widens the spinning-speed interval at which the system may perform rub and impact 


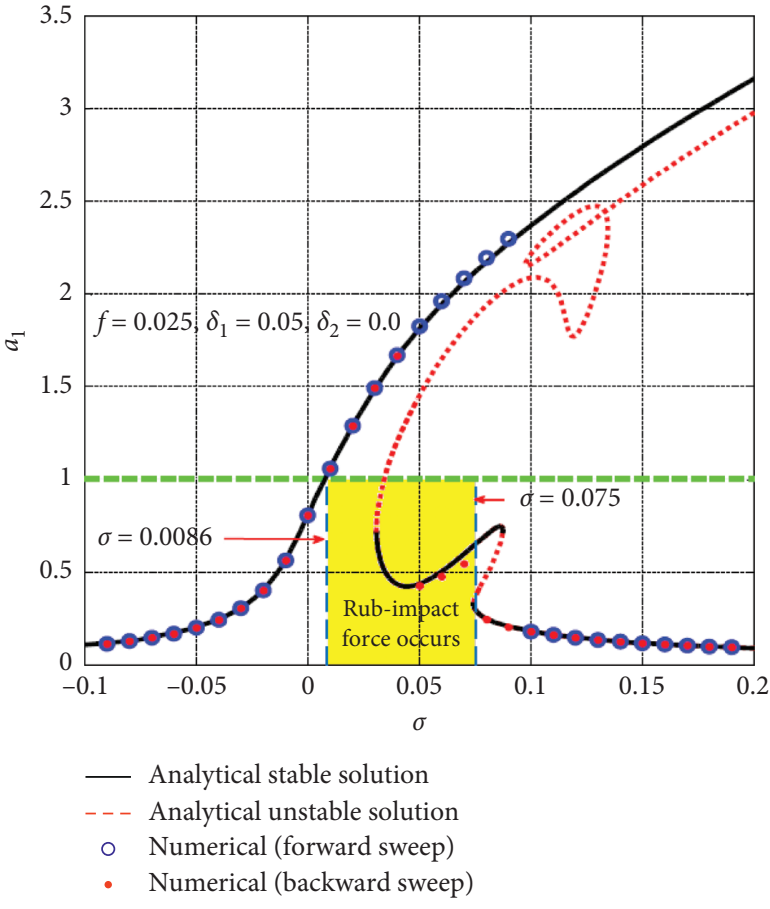

(a)

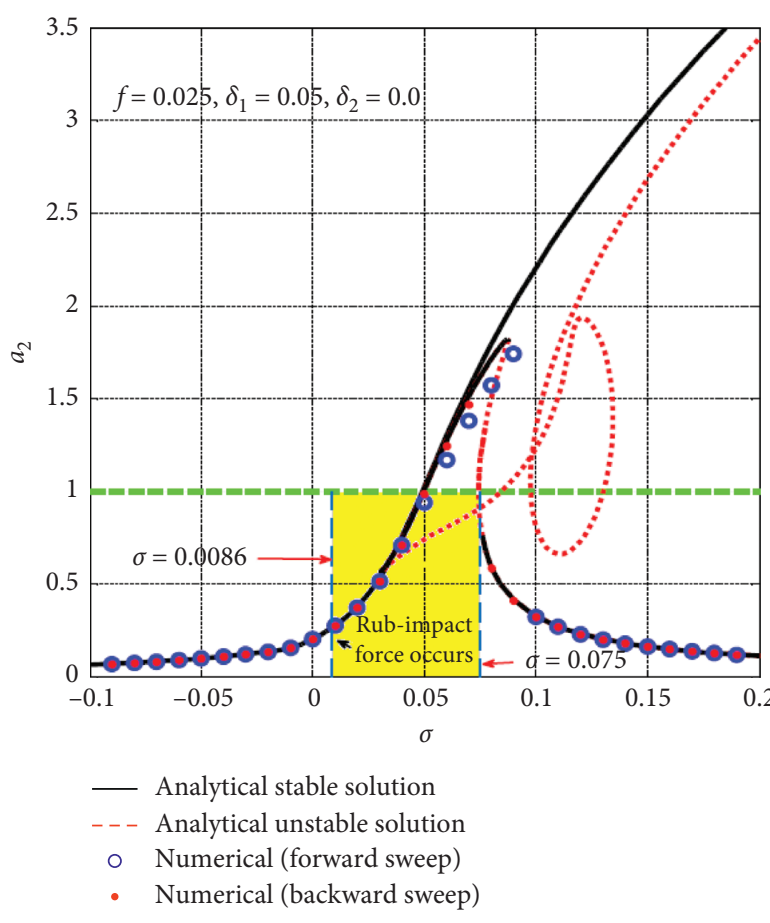

(b)

Figure 2: Spinning-speed response curve of the rotor system before control at $\delta_{1}=0.05, \delta_{2}=0.0$.

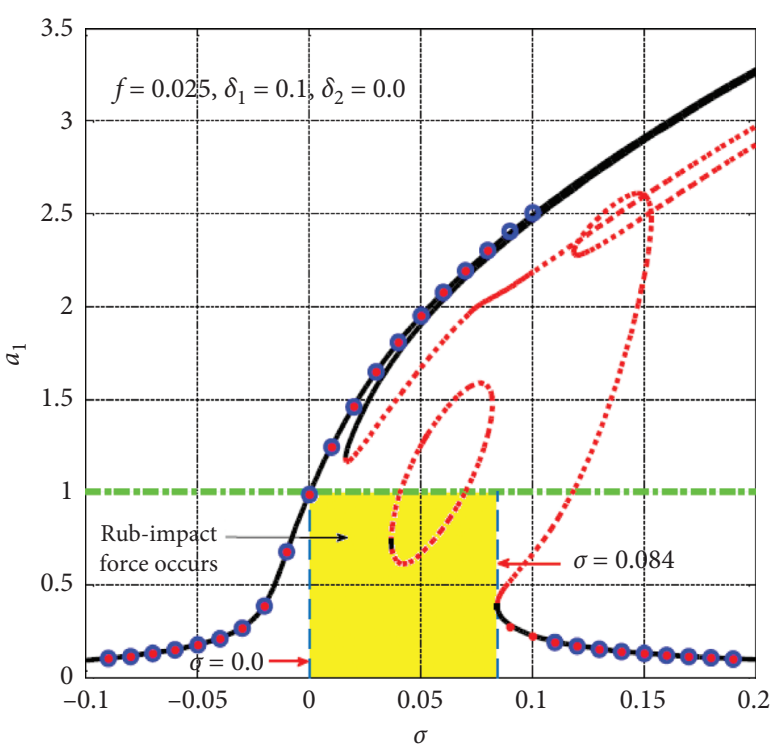

(a)

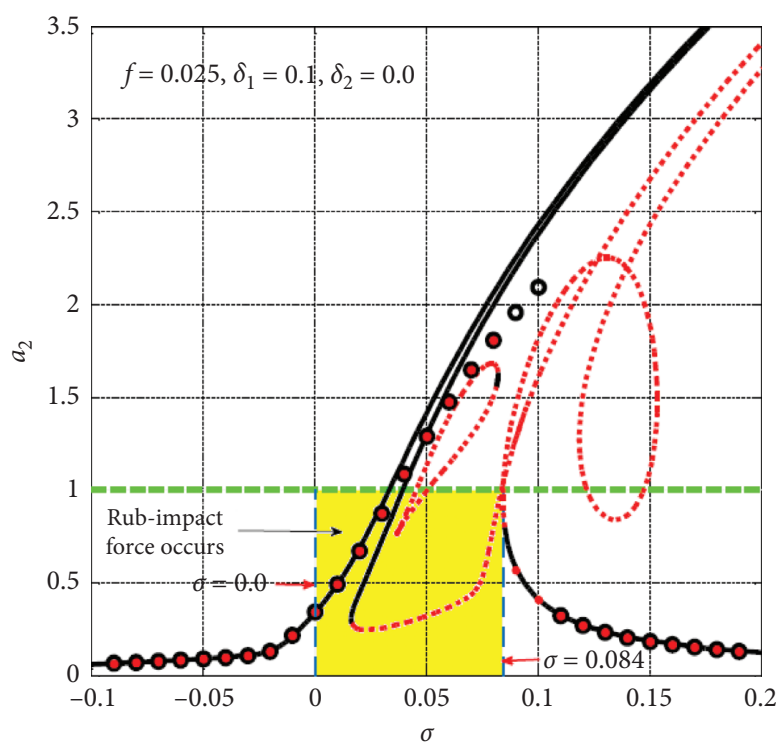

(b)

FIGURE 3: Spinning-speed response curve of the rotor system before control at $\delta_{1}=0.1, \delta_{2}=0.0$.

forces. In other words, Figure 2 shows that the system will oscillate with vibration amplitude higher than unity (i.e., $a_{1}>1$ and/or $a_{2}>1$ ) either in the horizontal or the vertical direction if $0.0086<\sigma<0.075$, while Figure 3 confirms that the system can oscillate with oscillation amplitude higher than unity (i.e., $a_{1}>1$ and/or $a_{2}>1$ ) if $0.0<\sigma<0.084$. Accordingly, the system performs rub and/or impact forces as long as $0.0086<\sigma<0.075$ if $\delta_{1}=0.05$ and $\delta_{2}=0.0$, but when $\delta_{1}=0.1$ and $\delta_{2}=0.0$, the system will suffer from rub and/or impact forces as long as $0.0<\sigma<0.084$.

To explore the system motion when the rub and impact occurs between the rotor and stator as explained above (i.e., when $a_{1} \geq 1$ and/or $a_{2} \geq 1$ ), the bifurcation diagram of the whole system model (i.e., equations (17a) and (17b) when the 
impact stiffness coefficient $\delta_{3}=0.5$ ) is obtained as shown in Figure 4, where Figure 4(a) shows the system bifurcation diagram according to Figure 2 (i.e., $\delta_{1}=0.05$ and $\delta_{2}=0.0$ ) and Figure 4 (b) depicts the system bifurcation diagram according to Figure 3 (i.e., $\delta_{1}=0.1$ and $\delta_{2}=0.0$ ). It is worth mentioning that the bifurcation diagrams in this article are obtained via plotting the Poincare return map of the steadystate radial displacement $R(\tau)=\sqrt{z_{1}(\tau)^{2}+z_{2}(\tau)^{2}}$ versus the targeted system parameter. Figure 4 shows that the system can perform periodic, quasiperiodic, or chaotic motion depending on both the magnitude of the linear asymmetric stiffness coefficient $\delta_{1}$ and the disk spinning speed $\Omega=\omega_{1}+\sigma$ when the rub and impact forces occurred. Although Figure 2 illustrates that the system suffers from rub and impact forces at $\sigma=0.05$, Figure 4(a) confirms that the system performs periodic motion at $\sigma=0.05$ depending on the obtained Poincare map. Accordingly, the radial temporal oscillation $R(\tau)$, orbit plot (i.e., $z_{1}(\tau)$ versus $z_{2}(\tau)$ ), and frequency spectrum for the asymmetric rotating disk are simulated in Figure 5 when $\sigma=0.05$ according to the bifurcation diagram given in Figure 4(a). By examining Figure 5 , it can be confirmed that the system exhibits periodic motion as is clear from Figure 5(a). At the same time, the system orbit plot in Figure 5(b) illustrates the occurrence of rub and impact forces.

By comparing Figures 3 and 4(b) at $\sigma=0.05$, it can be revealed that the system suffers from rub and impact forces as it is clear from Figure 3 (i.e., when $a_{1} \geq 1$ and/or $a_{2} \geq 1$ ). At the same time, Figure 4(b) illustrates that the system exhibits chaotic motion at $\sigma=0.05$. Accordingly, the radial temporal oscillation $R(\tau)$, orbit plot (i.e., $z_{1}(\tau)$ versus $z_{2}(\tau)$ ), and frequency spectrum for the asymmetric rotating disk are simulated in Figure 6 when $\sigma=0.05$ according to the bifurcation diagram given in Figure 4(b). The system exhibits a chaotic motion as shown in Figures 6(a) and 6(c). At the same time, the system orbit plot in Figure 6(b) illustrates the occurrence of rub and impact forces between the rotating disk and the magnetic poles legs.

The existence of both linear and nonlinear asymmetric stiffness (i.e., $\delta_{1}=\delta_{2} \neq 0$ ) on the lateral vibrations of the considered system is investigated in Figures 7 and 8, where Figure 7 illustrates the system spinning-speed response curves when $\delta_{1}=\delta_{2}=0.05$, while Figure 8 shows the spinning-speed response curves when $\delta_{1}=\delta_{2}=0.1$. Comparing Figures 7 and 8 with Figures 2 and 3, we can report that the asymmetry in both the linear and nonlinear stiffness coefficients may increase the system vibration amplitudes that result in widening the spinning-speed interval at which the rotor can perform rub and impact forces. It is clear from Figure 2 (i.e., $\delta_{1}=0.05, \delta_{2}=0$ ) that system can exhibit rub and impact forces as long as $0.0086<\sigma<0.075$, while Figure 7 (i.e., $\delta_{1}=\delta_{2}=0.05$ ) illustrates that the system will perform rub and impact as long as $0.01<\sigma<0.085$. Also, Figure 3 (i.e., $\delta_{1}=0.1, \delta_{2}=0$ ) shows that the system can exhibit rub and impact as long as $0.0<\sigma<0.084$, while Figure 8 (i.e., $\delta_{1}=\delta_{2}=0.1$ ) confirms that the rotor will exhibits rub and impact as long as $0.0<\sigma<0.1$.
According to Figures 7 and 8, the rotor bifurcation diagrams are illustrated as shown in Figure 9 via solving equations (17a) and (17b) numerically when $\delta_{3}=5.0$. Figure 9(a) depicts the system bifurcation diagram according to Figure 7 (i.e., when $\delta_{1}=\delta_{2}=0.05$ ), while Figure 9(b) shows the bifurcation diagram according to Figure 8 (i.e., when $\delta_{1}=\delta_{2}=0.1$ ). It is clear from Figure 9 that the system can perform either periodic or nonperiodic motion as long as the rub and impact forces between the rotor and stator occur (i.e., when $0.01<\sigma<0.085$ regarding Figure 9(a) and $0.0<\sigma<0.1$ regarding Figure 9(b)), but the system oscillates periodically when the rum/impact force disappeared. Numerical simulations for the system temporal oscillation and the corresponding whirling orbit according to Figure 9(b) when $\sigma=0.025$ and 0.05 are illustrated in Figures 10 and 11, respectively. Figure 10 confirms that the system performs periodic motion when $\sigma=0.025$, while Figure 11 exhibits chaotic motion when $\sigma=0.05$.

4.2. Rub and Impact Forces of the System with Control. The efficiency of the proposed control strategy in suppressing rotor lateral vibrations and eliminating the rubimpact forces is discussed within this subsection. The system spinning-speed response curves at three different values of the asymmetric stiffness coefficients $\left(\delta_{1}\right.$ and $\left.\delta_{2}\right)$ are illustrated in Figure 12 when the proportional gain $p=1.0$ and the derivative control gain $d=0.025$. Figures $12(\mathrm{a})$ and 12(b) show the system response curves at $\delta_{1}=\delta_{2}=0.05,075$, while Figures $12(\mathrm{c})$ and $12(\mathrm{~d})$ illustrate the controlled system response curves at $\delta_{1}=\delta_{2}=0.1$. Figures 12(a) and 12(b) prove that the applied controller has mitigated the system oscillation amplitudes below unity (i.e., $a_{1}<1$ and $a_{2}<1$ ) when $\delta_{1}=\delta_{2}=0.05$, which ultimately prevents the rub and impact forces. Accordingly, it can be emphasized that the asymmetric system with asymmetric coefficients $\delta_{1}=\delta_{2}=0.05$ that has strong nonlinear characteristics as illustrated in Figure 7 can be controlled very well to respond as a linear system utilizing the applied controller with proportional control gain $p=1.0$ and derivative control gain $d=0.025$. However, Figures 12 (a) and 12(b) illustrate that the increase of $\delta_{1}$ and $\delta_{2}$ from $\delta_{1}=\delta_{2}=$ 0.05 to $\delta_{1}=\delta_{2}=0.075$ has increased the controlled system oscillation amplitudes $a_{1}$ and $a_{2}$ to become higher than unity at the spinning-speed interval $0.02<\sigma<0.078$, which means that the occurrence of rub and impact forces when the controller is turned on with control gains $p=1.0$ and $d=0.025$. Also, Figures $12(\mathrm{c})$ and $12(\mathrm{~d})$ confirm that the increase of $\delta_{1}$ and $\delta_{2}$ to become $\delta_{1}=\delta_{2}=0.15$ makes the controlled system (i.e., $p=1.0$ and $d=0.025$ ) respond as a strong nonlinear one again with high oscillation amplitudes where the rub and impact occur at the spinning-speed interval $-0.003<\sigma<0.11$.

Depending on the obtained spinning-speed response curves in Figure 12, the system bifurcation diagrams are simulated as shown in Figure 13, where Figure 13(a) depicts the system bifurcation diagram according to Figures 12(a) and 12(b) when $\delta_{1}=\delta_{2}=0.075$, and Figure 13(b) shows the 


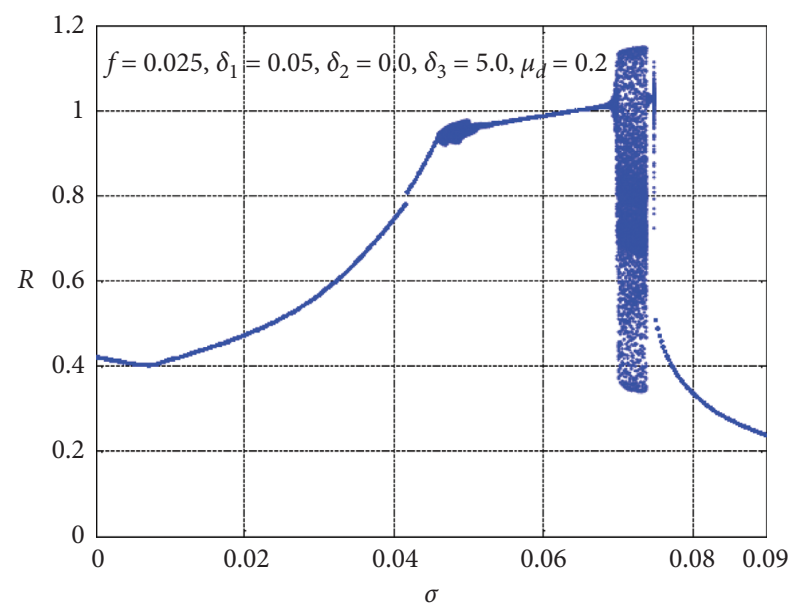

(a)

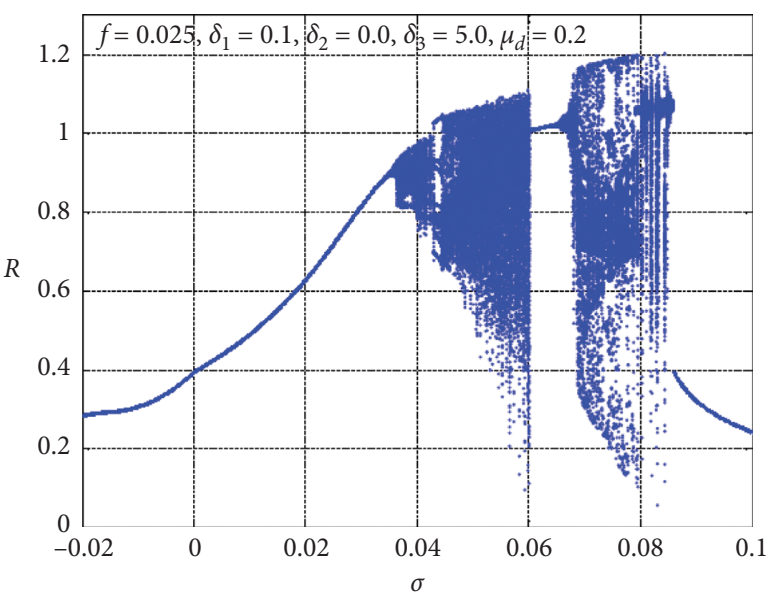

(b)

Figure 4: Bifurcation diagram of the system before control: (a) bifurcation diagram according to Figure 2 (i.e., when $\delta_{1}=0.05, \delta_{2}=0.0$ ); (b) bifurcation diagram according to Figure 3 (i.e., when $\delta_{1}=0.1, \delta_{2}=0.0$ ).

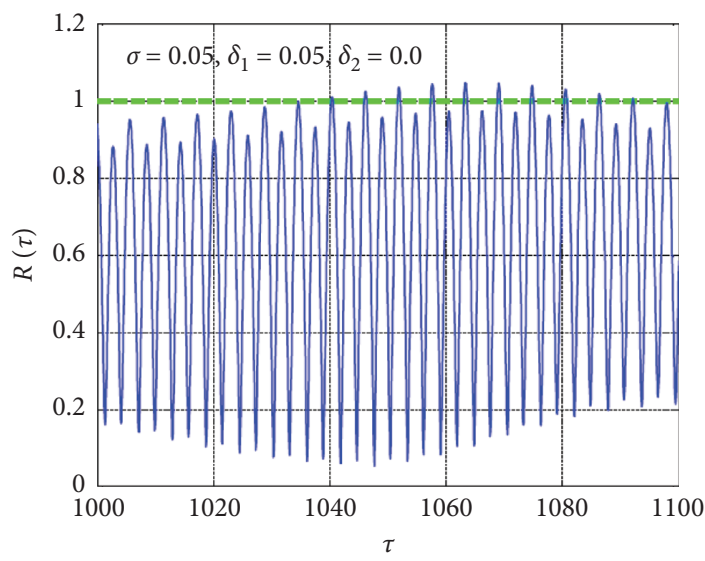

(a)

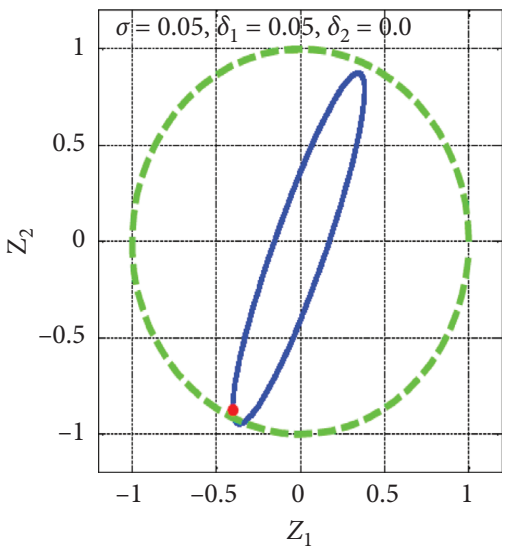

(b)

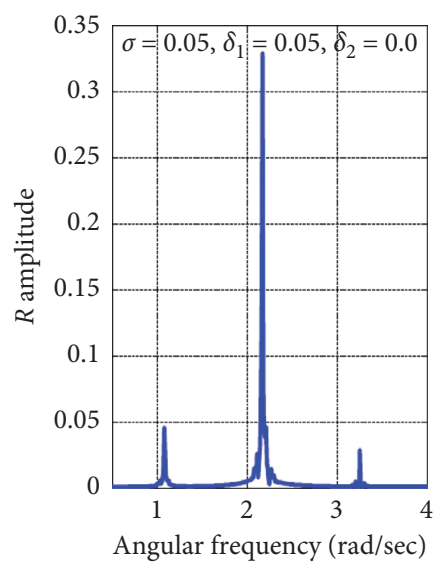

(c)

FIGURE 5: Uncontrolled system time response according to Figure 4(a) (i.e., at $\delta_{1}=0.05, \delta_{2}=0.0$ ) when $\sigma=0.05$ : (a) the temporal oscillation of the radial displacement $R(\tau)=\sqrt{z_{1}^{2}+z_{2}^{2}}$, (b) whirling orbit $z_{1}(\tau)$ versus $z_{2}(\tau)$, and (c) frequency spectrum.

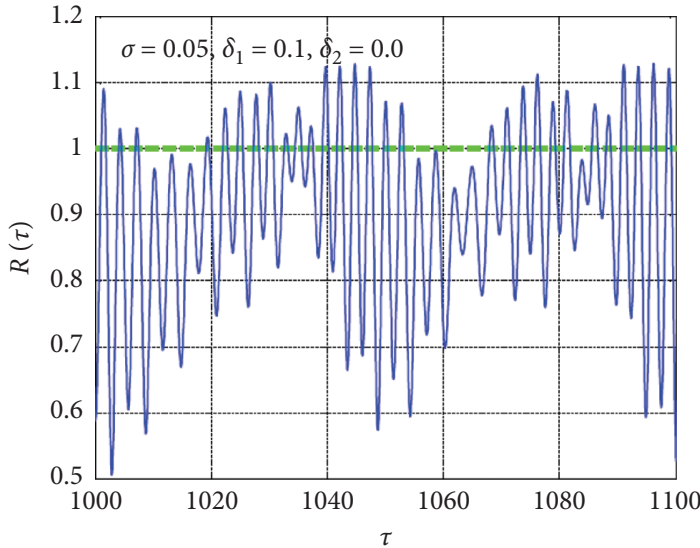

(a)

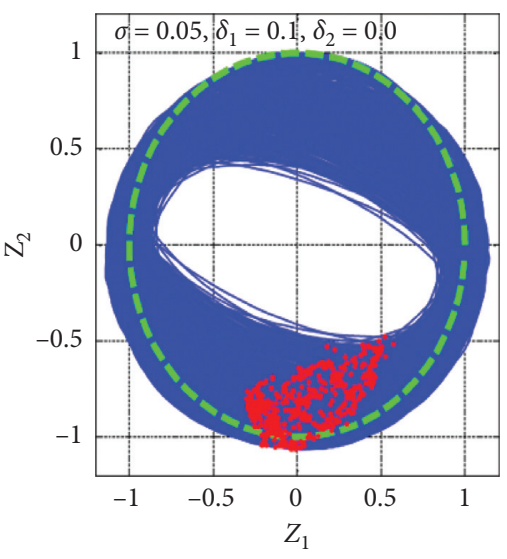

(b)

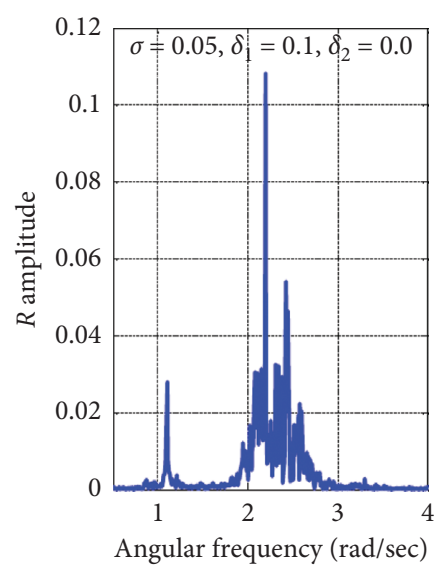

(c)

Figure 6: Uncontrolled system time response according to Figure 4(b) (i.e., at $\delta_{1}=0.1, \delta_{2}=0.0$ ) when $\sigma=0.05$ : (a) the temporal oscillation of the radial displacement $R(\tau)=\sqrt{z_{1}^{2}+z_{2}^{2}}$, (b) whirling orbit $z_{1}(\tau)$ versus $z_{2}(\tau)$, and (c) frequency spectrum. 


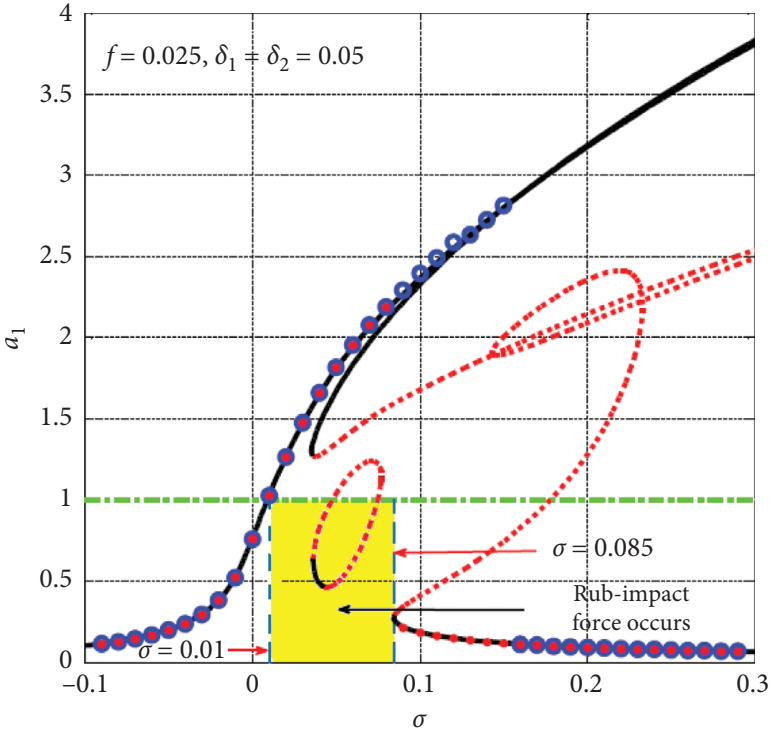

(a)

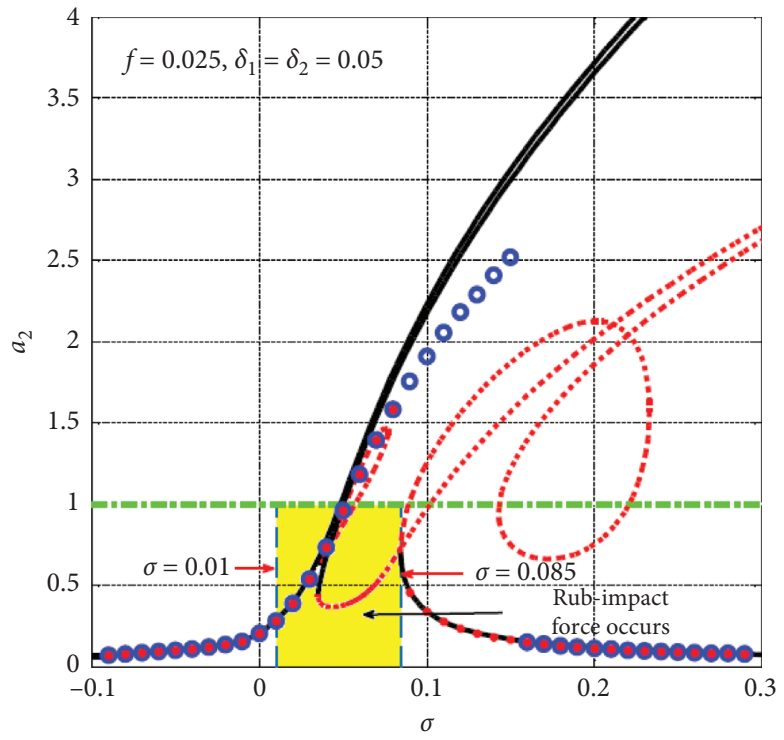

(b)

FIGURE 7: Spinning-speed response curve of the rotor system before control at $\delta_{1}=\delta_{2}=0.05$.

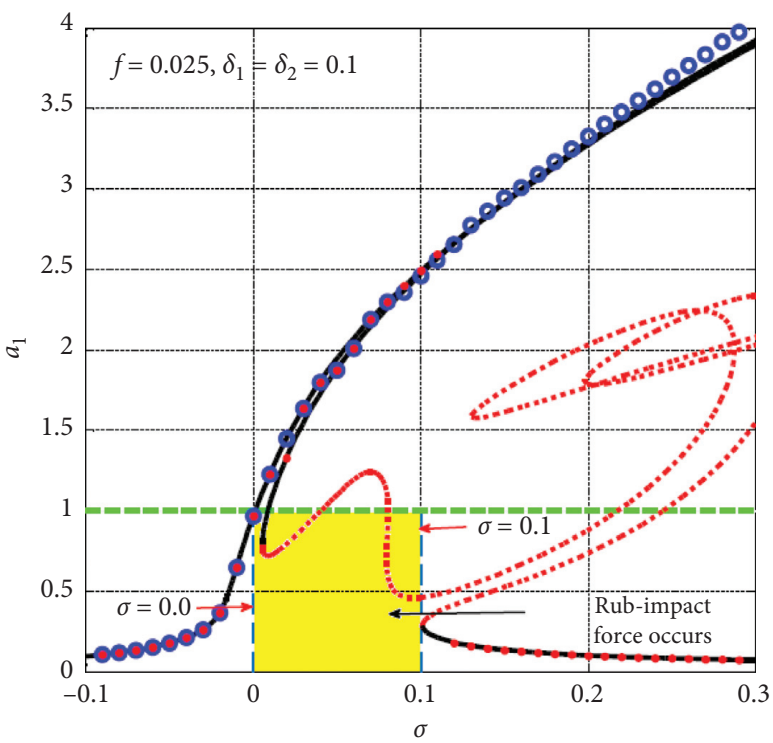

(a)

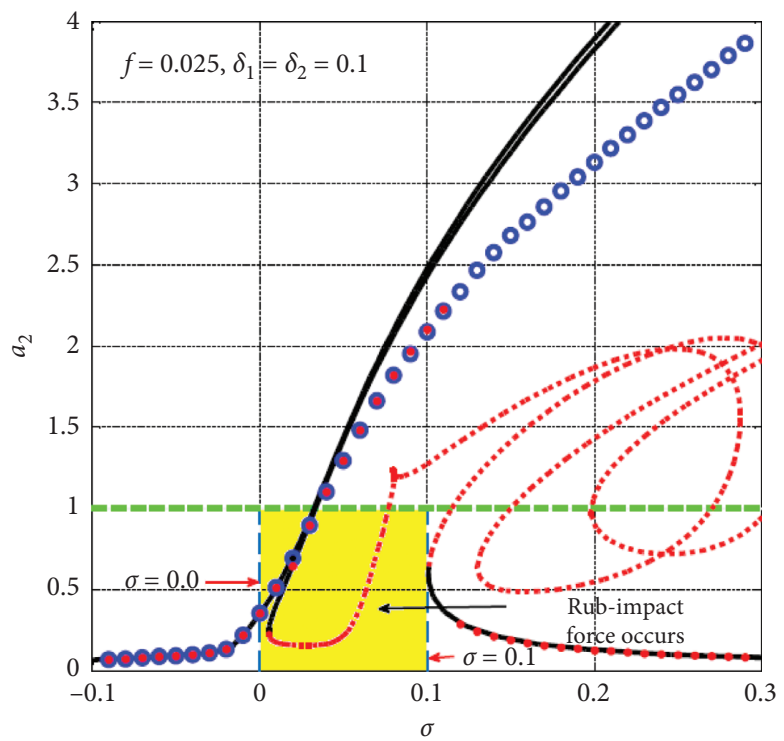

(b)

FIgURE 8: Spinning-speed response curve of the rotor system before control at $\delta_{1}=\delta_{2}=0.1$.

rotating shaft bifurcation diagram according to Figures 12(c) and $12(\mathrm{~d})$ when $\delta_{1}=\delta_{2}=0.15$. Generally, Figure 13 illustrates that the asymmetric system can perform periodic, quasiperiodic, chaotic, or n-period motion depending on both the angular speed $\Omega=\omega_{1}+\sigma$ and the asymmetric stiffness coefficients $\left(\delta_{1}\right.$ and $\left.\delta_{2}\right)$ even if the controller was turned on.

Based on Figure 13, numerical simulations for the rotor radial displacement and whirling orbit are illustrated in Figures 14 to 16, where Figure 14 shows the system temporal oscillation according to Figure 13 at $\sigma=0.04$ and Figures 15 and 16 illustrate the system temporal oscillation according to Figure 14 at $\sigma=0.06,0.085$. It is clear from Figure 14 that the system can oscillate with quasiperiodic motion, while Figures 15 and 16 confirm the oscillation of the system with chaotic and 10-period motions depending on the disk spinning-speed $\Omega=\omega_{1}+\sigma$.

Figure 12 shows the failure of the applied controller in suppressing the rub and impact forces with the control gains $p=1.0$ and $d=0.025$ when $\delta_{1}=\delta_{2}>0.05$, and the effect of increasing the derivative control gain from $d=0.025$ to $d=$ 0.1 is illustrated in Figure 17 when $\delta_{1}=\delta_{2}=0.15$ at 


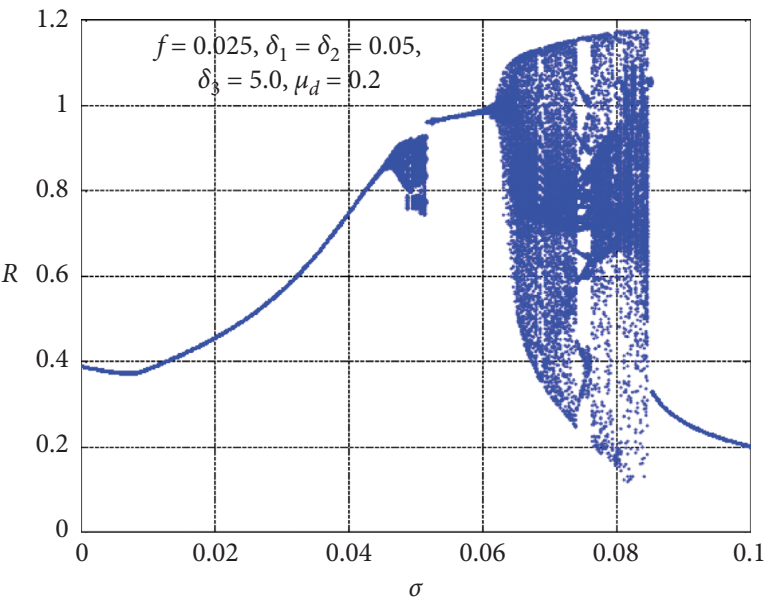

(a)

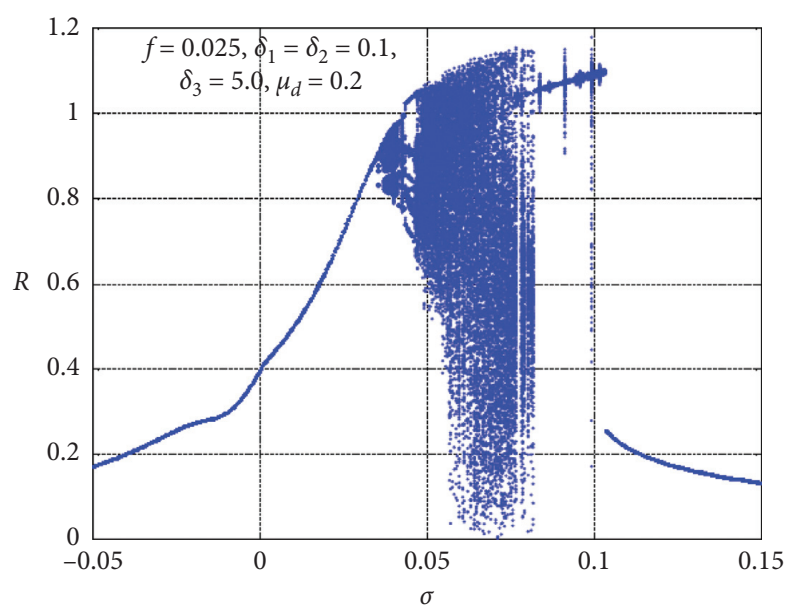

(b)

FIGURE 9: Bifurcation diagram of the uncontrolled system: (a) bifurcation diagram according to Figure 7 (i.e., when $\delta_{1}=\delta_{2}=0.05$ ); (b) bifurcation diagram according to Figure 8 (i.e., when $\delta_{1}=\delta_{2}=0.1$ ).

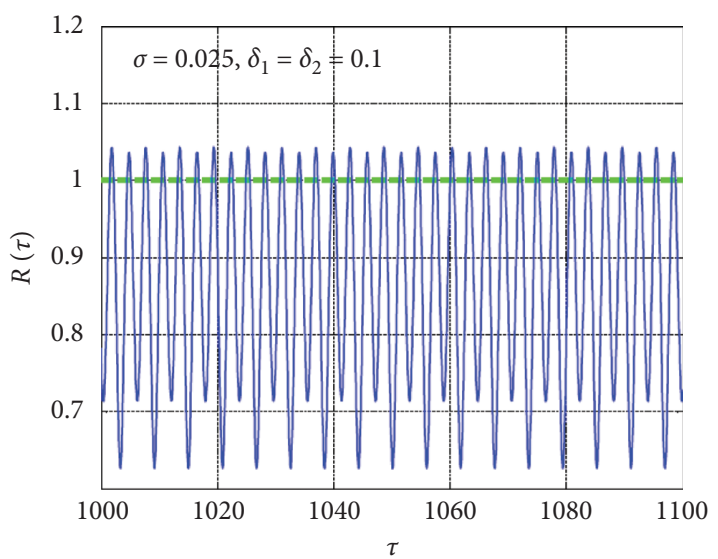

(a)

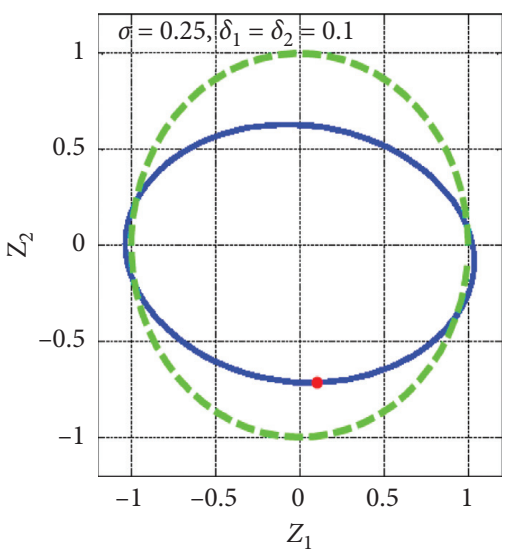

(b)

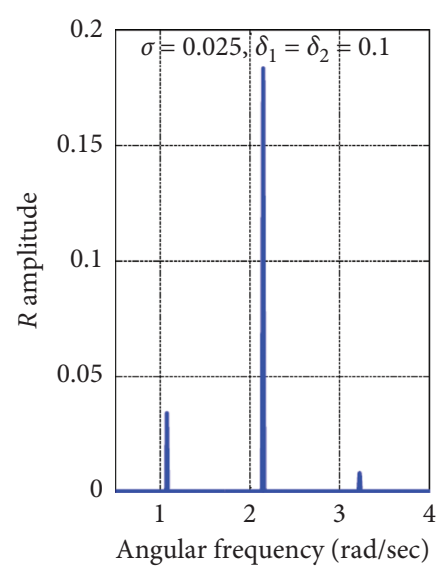

(c)

Figure 10: Uncontrolled system time response according to Figure 9(a) (i.e., at $\delta_{1}=\delta_{2}=0.05$ ) when $\sigma=0.025$ : (a) the temporal oscillation of the radial displacement $R(\tau)=\sqrt{z_{1}^{2}+z_{2}^{2}}$, (b) whirling orbit $z_{1}(\tau)$ versus $z_{2}(\tau)$, and (c) frequency spectrum.

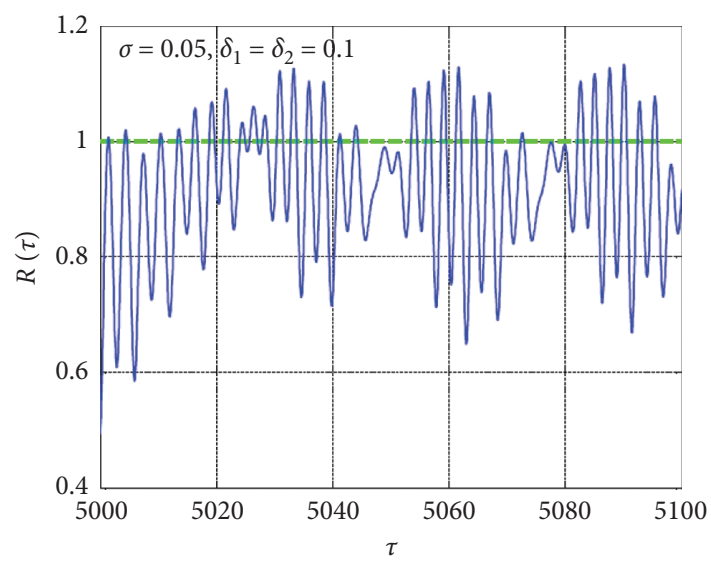

(a)

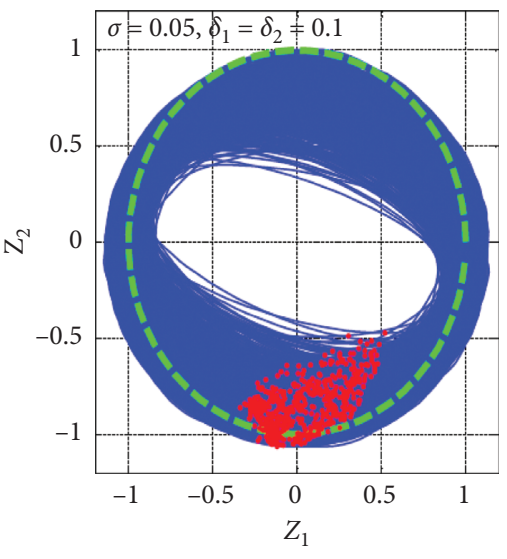

(b)

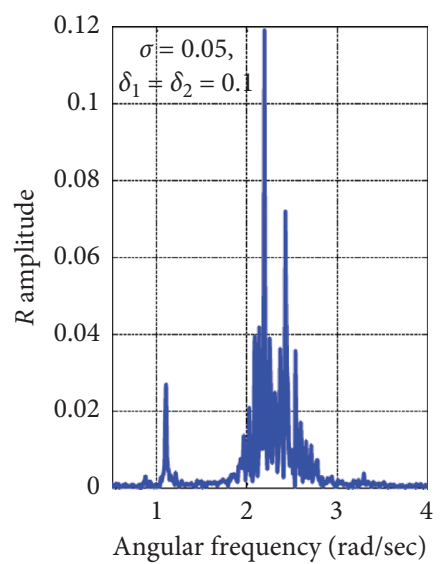

(c)

FIGURE 11: Uncontrolled system time response according to Figure 9(b) (i.e., at $\delta_{1}=\delta_{2}=0.1$ ) when $\sigma=0.05$ : (a) the temporal oscillation of the radial displacement $R(\tau)=\sqrt{z_{1}^{2}+z_{2}^{2}}$, (b) whirling orbit $z_{1}(\tau)$ versus $z_{2}(\tau)$, and (c) frequency spectrum. 


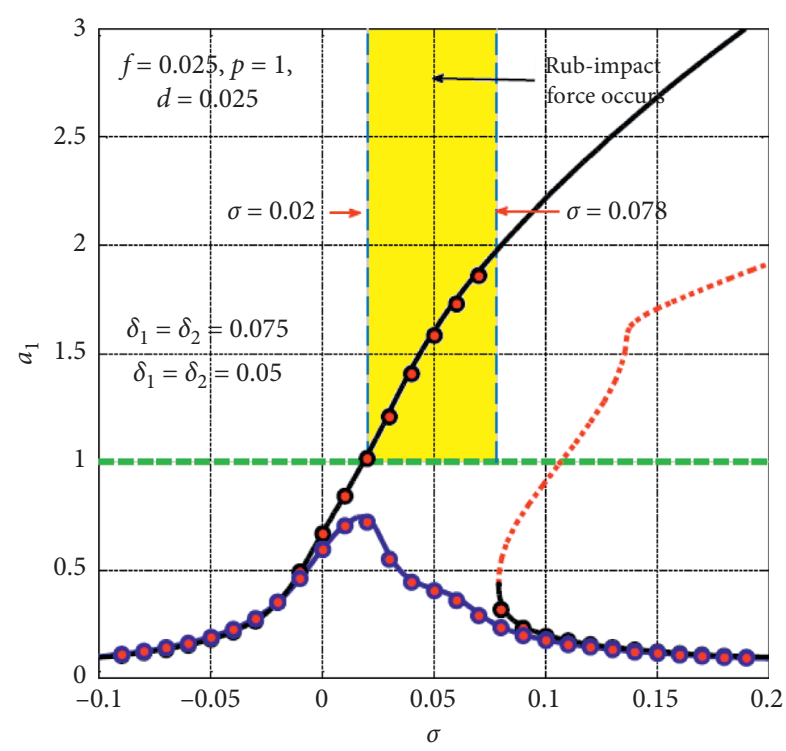

(a)

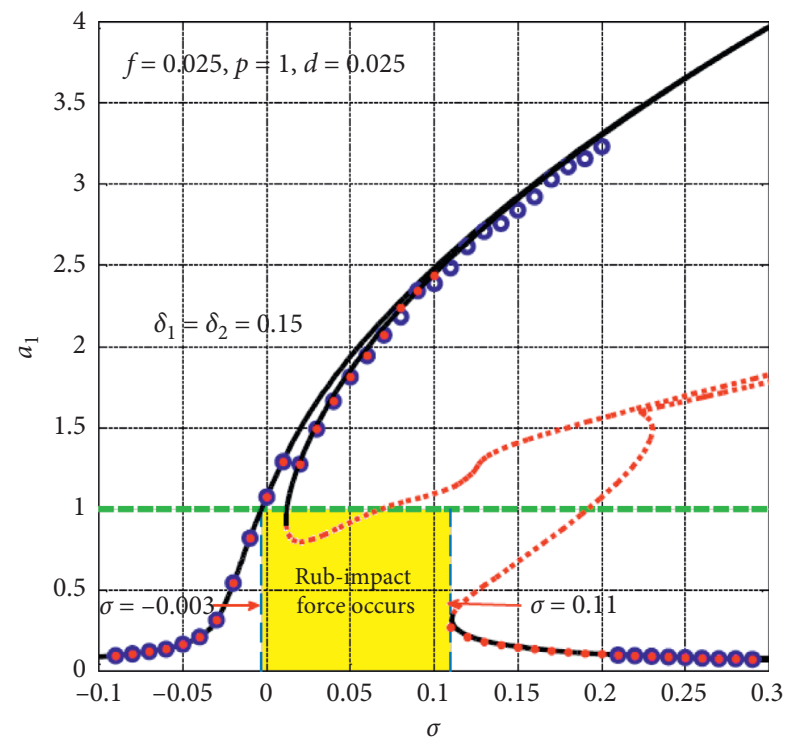

(c)

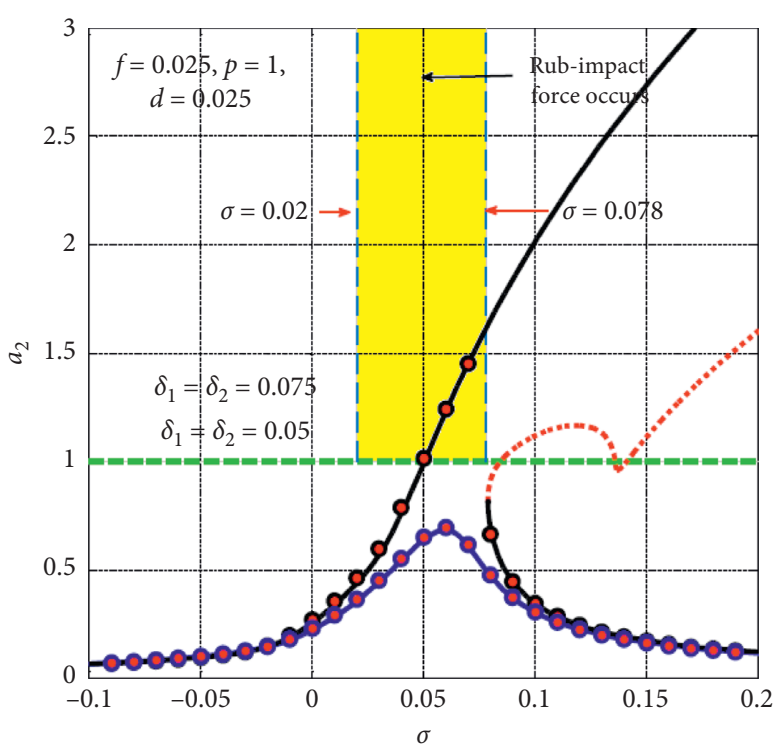

(b)

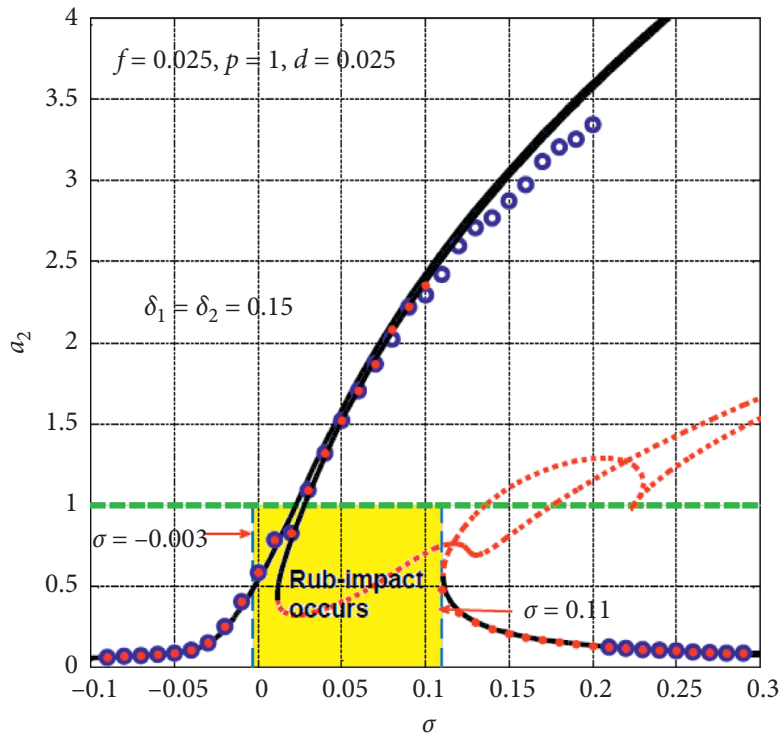

(d)

Figure 12: Controlled system spinning-speed response curves when the proportional control gain $p=1.0$ and derivative control gain $d=0.025$ at three different values of the linear and nonlinear asymmetric stiffness coefficient (i.e., $\left.\delta_{1}=\delta_{2}=0.05,0.075,0.15\right)$ : $(\mathrm{a}, \mathrm{b})$ spinning-speed response curves when $\delta_{1}=\delta_{2}=0.05,0.1$ and (c, d) spinning-speed response curves when $\delta_{1}=\delta_{2}=0.15$.

$f=0.025,0.05$. Figure 17 confirms that the applied control law can suppress the system oscillation amplitudes in a way such that $a_{1}<1$ and $a_{2}<1$ even at the large asymmetric stiffness coefficients (i.e., $\delta_{1}=\delta_{2}=0.15$ ). In addition, the system responds as a linear system without rub and impact forces occurrence. To determine the boundaries of the asymmetric stiffness at which the system will start to perform rub and impact forces, the system oscillation amplitudes $a_{1}$ and $a_{2}$ are plotted versus $\delta_{1}=\delta_{2}$ when $p=1.0$, $d=0.1$, and $f=0.025$ as shown in Figure 18. Figures 18(a) and $18(\mathrm{~b})$ show $\delta_{1}=\delta_{2}$ response curve when $\sigma=0$, and Figures 18(c) and 18(d) depict $\delta_{1}=\delta_{2}$ response curve when $\sigma=0.05$. Figures 18 (a) and 18 (b) confirm that the controlled system can operate safely without rub-impact as long as $\delta_{1}=\delta_{2}<0.29$, while Figures 18(c) and 18(d) illustrate that the controlled rotor can work safely without rub-impact as long as $\delta_{1}=\delta_{2}<0.1977$.

The controlled system bifurcation diagram utilizing $\delta_{1}=$ $\delta_{2}$ as the bifurcation control parameter is obtained in Figure 19 depending on Figure 18. Figure 19(a) shows the bifurcation diagram according to Figures 18(a) and 18(b) (i.e., when $\sigma=0$ ), while Figure 19(b) shows the system bifurcation diagram according to Figures 18(c) and 18(d) (i.e., when $\sigma=0.05$ ). Based on the bifurcation diagrams shown in Figure 19, it can be deduced that the controlled system can oscillate with periodic, quasiperiodic, chaotic, or period-n motion depending on the magnitudes of $\delta_{1}, \delta_{2}$, and $\sigma$. Numerical simulation for the system temporal oscillation 


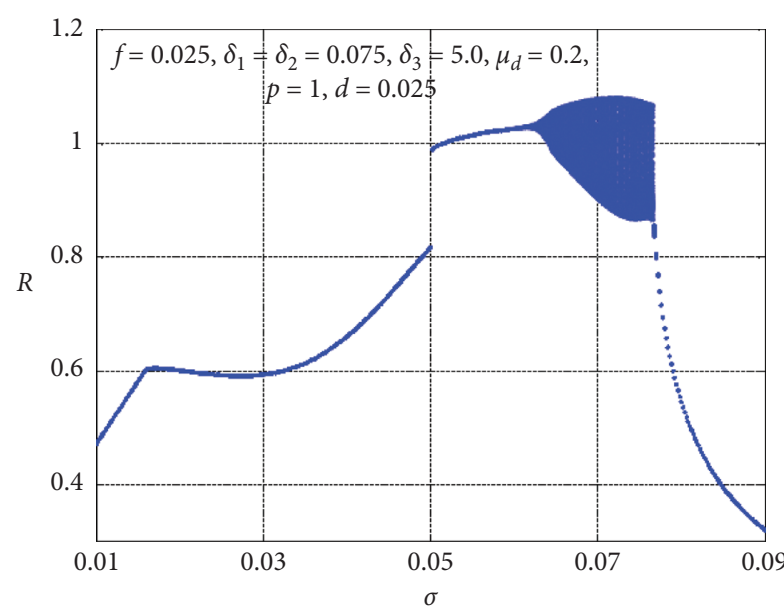

(a)

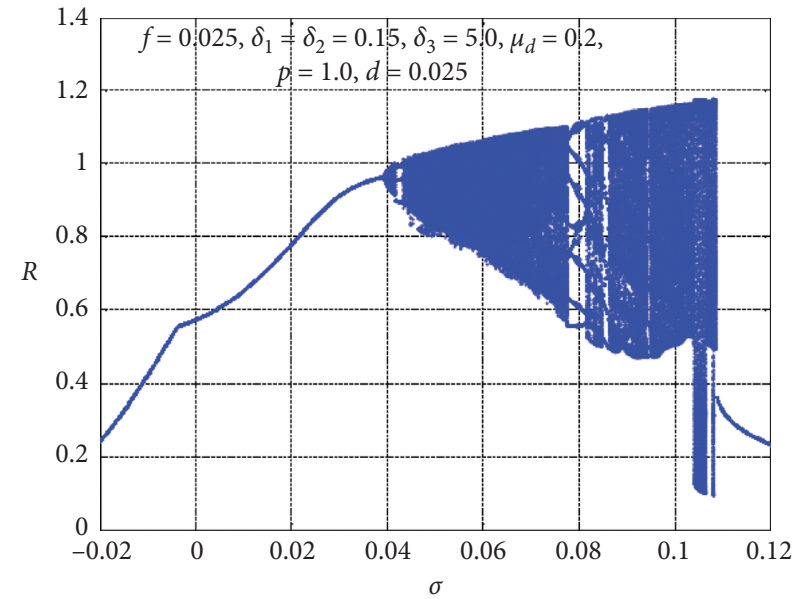

(b)

FiguRE 13: Bifurcation diagram of the controlled system (i.e., $p=1.0 d=0.025$ ): (a) bifurcation diagram according to Figures 12(a) and 12(b) when $\delta_{1}=\delta_{2}=0.075$; (b) bifurcation diagram according to Figures 12(c) and 12(d) (i.e., when $\delta_{1}=\delta_{2}=0.15$ ).

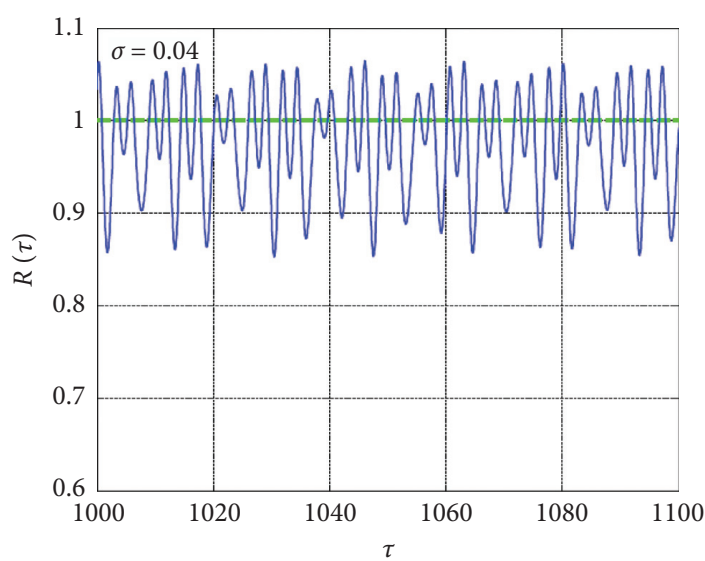

(a)

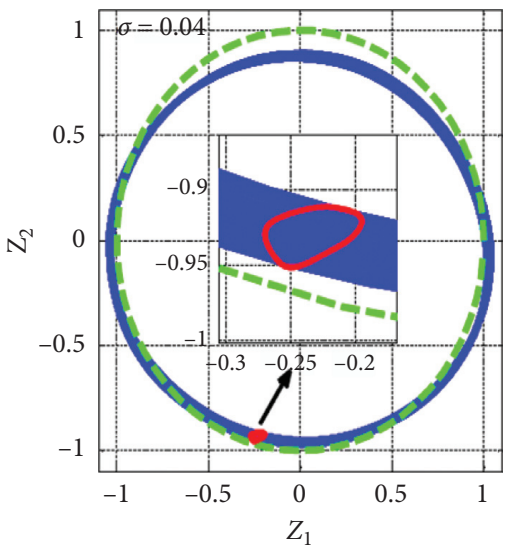

(b)

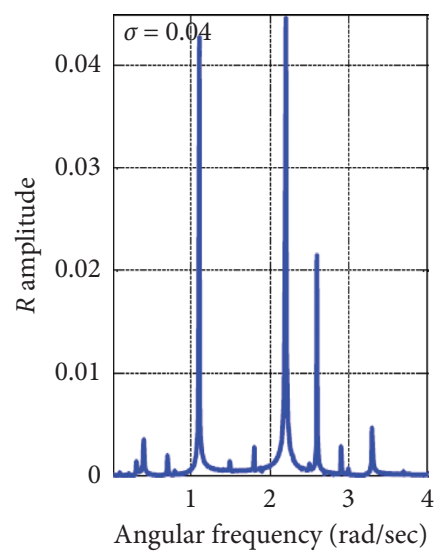

(c)

Figure 14: Controlled system time response (i.e., $p=1.0, d=0.025$ ) according to Figure 13(a) when $\delta_{1}=\delta_{2}=0.075$ at $\sigma=0.04$ : (a) the temporal oscillation of the radial displacement $R(\tau)=\sqrt{z_{1}^{2}+z_{2}^{2}}$, (b) whirling orbit $z_{1}(\tau)$ versus $z_{2}(\tau)$, and (c) frequency spectrum.

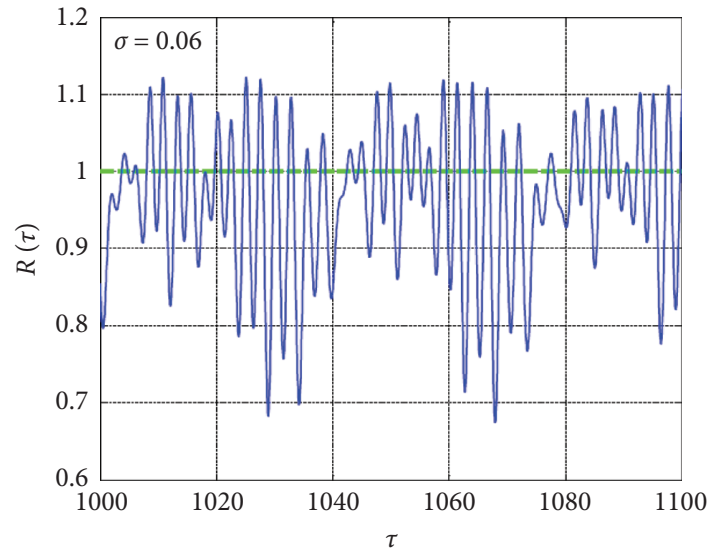

(a)

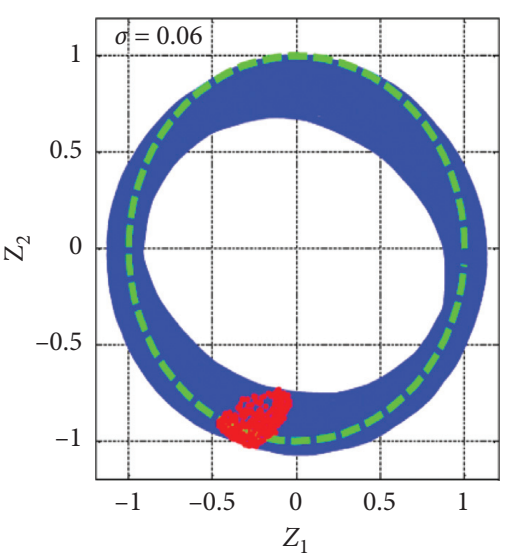

(b)

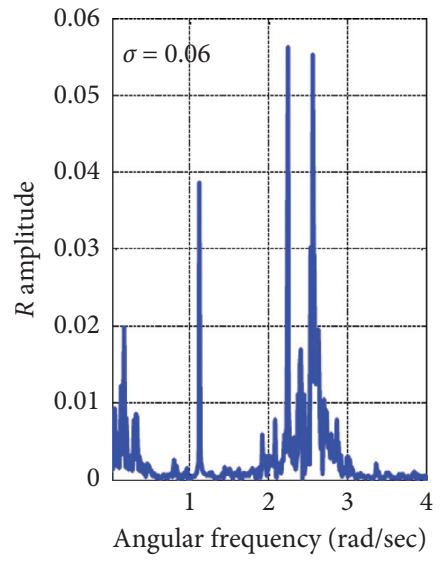

(c)

Figure 15: Controlled system time response (i.e., $p=1.0, d=0.025)$ according to Figure 13(b) when $\delta_{1}=\delta_{2}=0.15$ at $\sigma=0.06$ : (a) the temporal oscillation of the radial displacement $R(\tau)=\sqrt{z_{1}^{2}+z_{2}^{2}}$, (b) whirling orbit $z_{1}(\tau)$ versus $z_{2}(\tau)$, and (c) frequency spectrum. 


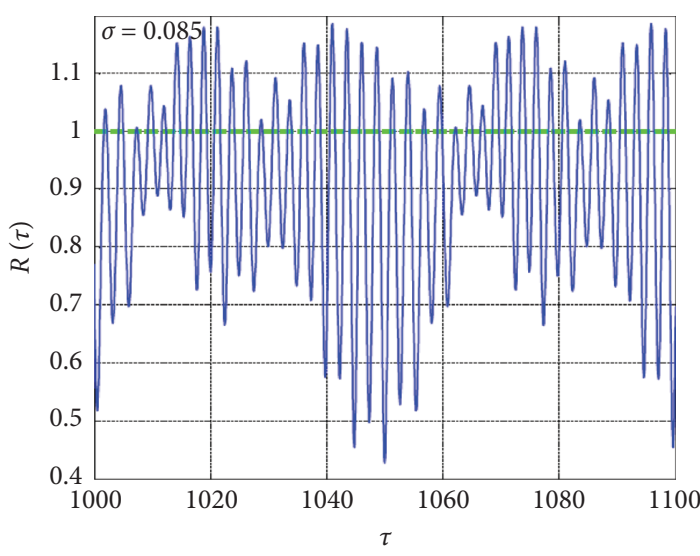

(a)

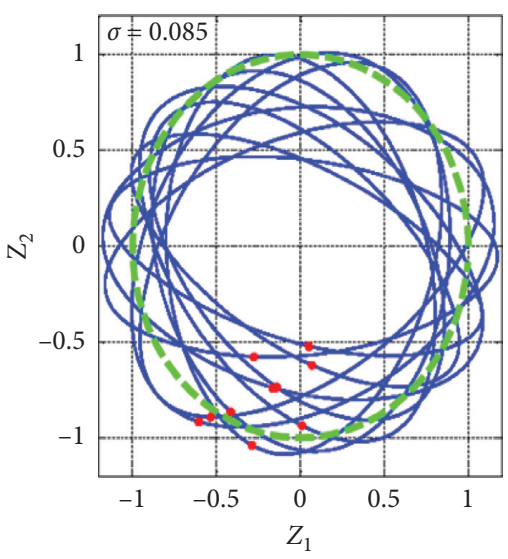

(b)

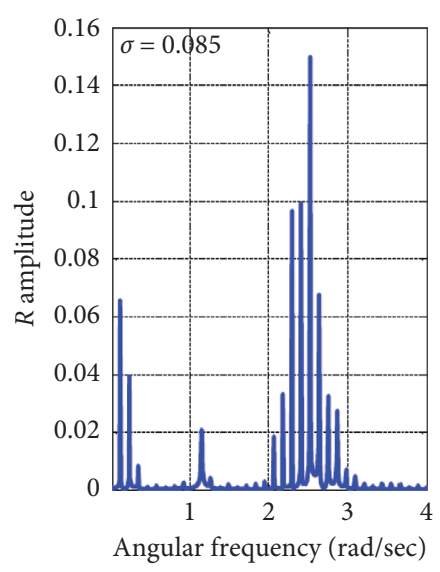

(c)

Figure 16: Controlled system time response (i.e., $p=1.0, d=0.025)$ according to Figure $13(\mathrm{~b})$ when $\delta_{1}=\delta_{2}=0.15$ at $\sigma=0.085$ : (a) the temporal oscillation of the radial displacement $R(\tau)=\sqrt{z_{1}^{2}+z_{2}^{2}}$, (b) whirling orbit $z_{1}(\tau)$ versus $z_{2}(\tau)$, and (c) frequency spectrum.

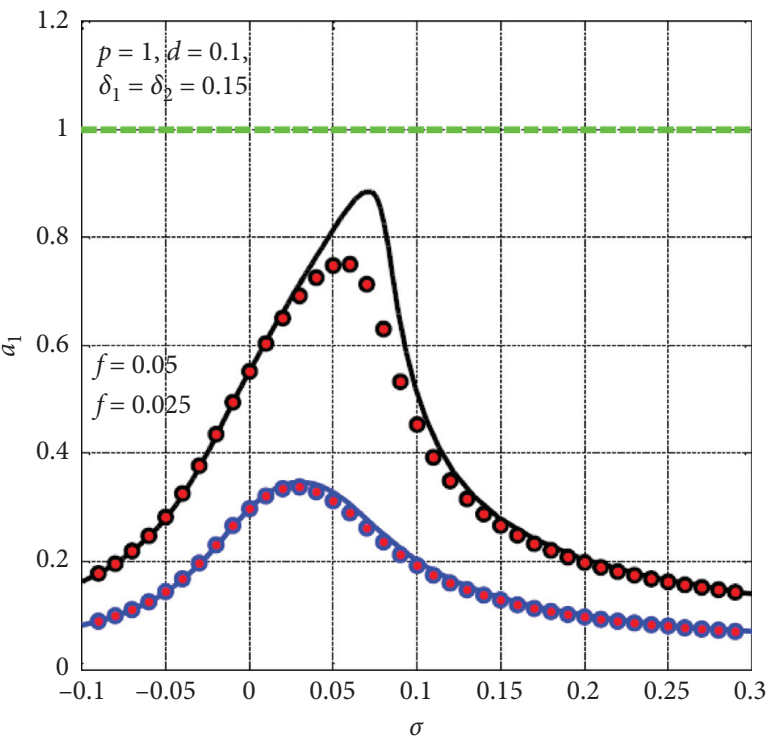

(a)

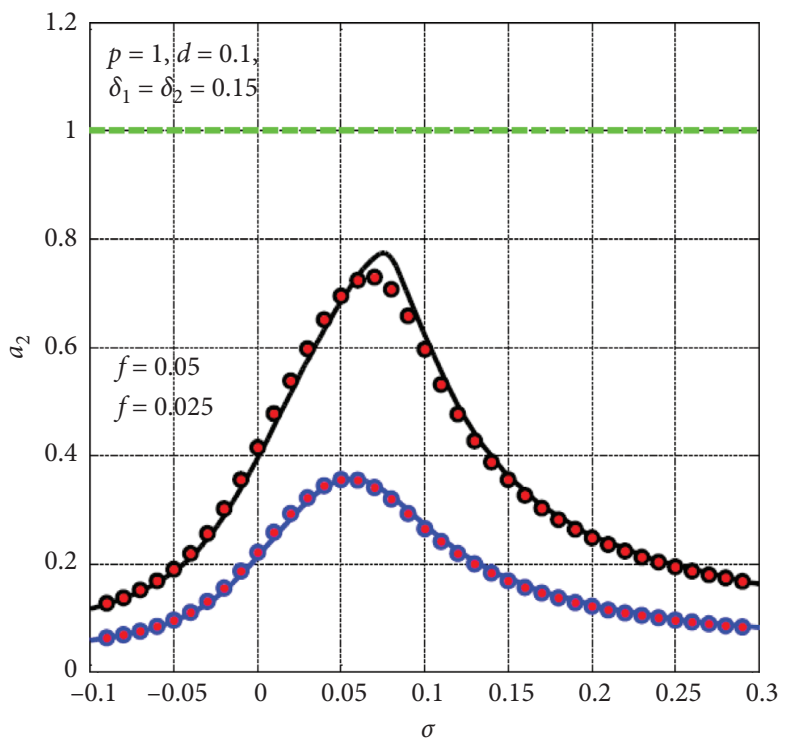

(b)

FIGURE 17: Controlled system spinning-speed response curves when the proportional control gain $p=1.0$ and derivative control gain $d=0.1$ at two different values of the disk eccentricity $f$ when $\delta_{1}=\delta_{2}=0.15$.

and whirling motion according to Figure 19(b) (i.e., $\left.p=1.0, d=0.1, \sigma=0.05, f=0.025, \delta_{3}=5\right)$ at three different values of $\delta_{1}=\delta_{2}$ is illustrated in Figures 20-22. Figure 20 shows the system period-2 motion when $\delta_{1}=\delta_{2}=0.3$, and Figure 21 confirms the system period-5 motion when $\delta_{1}=\delta_{2}=0.45$, while Figure 22 depicts the system quasiperiodic motion when $\delta_{1}=\delta_{2}=0.5$.

The influence of increasing $f$ on the dynamical behaviours and the nature of motion (i.e., $p=1.0$ and $d=0.1$ ) is illustrated in Figures 23 and 24, utilizing $f$ as the main bifurcation control parameter. Figures 23(a) and 23(b) illustrate the system eccentricity response curves when $\delta_{1}=\delta_{2}=0.15, \sigma=0$, while Figures 23(c) and 23(d) depict the eccentricity response curves when $\delta_{1}=\delta_{2}=0.15, \sigma=0.05$. By investigating the figure, we can deduce that the controlled rotor may be subject to rub and impact forces if $f>0.12$ at $\sigma=0$, but the system will exhibit rub and impact force at $\sigma=0.05$ if the disk eccentricity $f>0.064$. According to Figure 23, the system bifurcation diagrams are depicted in Figure 24 employing the disk eccentricity $f$ as the bifurcation parameter. Figure 24(a) shows the bifurcation diagram according to Figures 23(a) and 23(b) (i.e., when $p=1.0, d=0.1, \delta_{1}=\delta_{2}=0.15, \sigma=0.0$ ), while Figure 24(b) depicts the bifurcation diagram according to Figures 23(c) and 23(d) (i.e., when $p=1.0, d=0.1, \delta_{1}=\delta_{2}=0.15 . \sigma=0.05$ ). Comparing Figures 23 and 24, we can deduce that the controlled system can exhibit periodic oscillation only as long as the rub and impact forces between the rotor and stator do not occur (i.e., as long as $f>0.12$ as in Figure 24(a) or as long as $f>0.064$ as in 


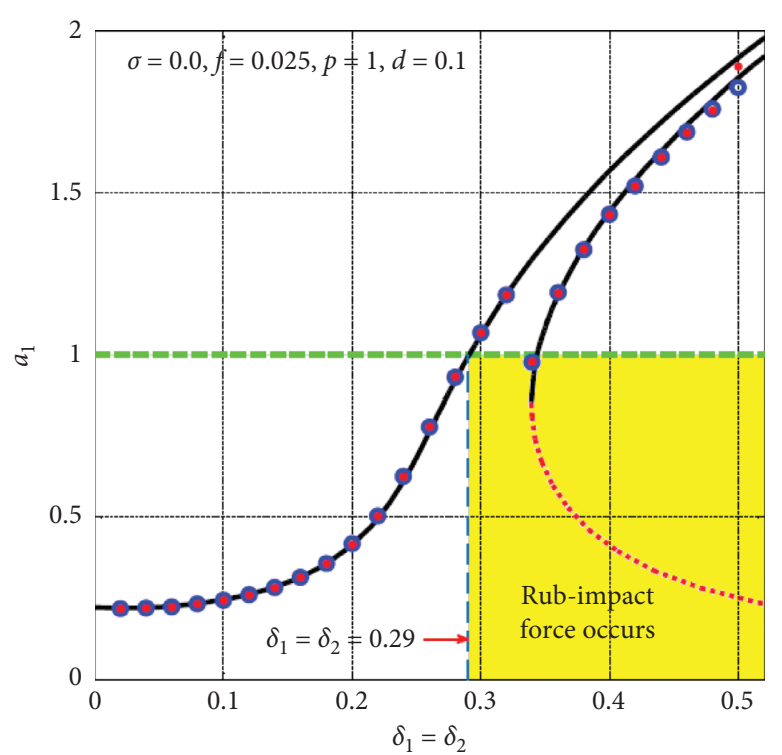

(a)

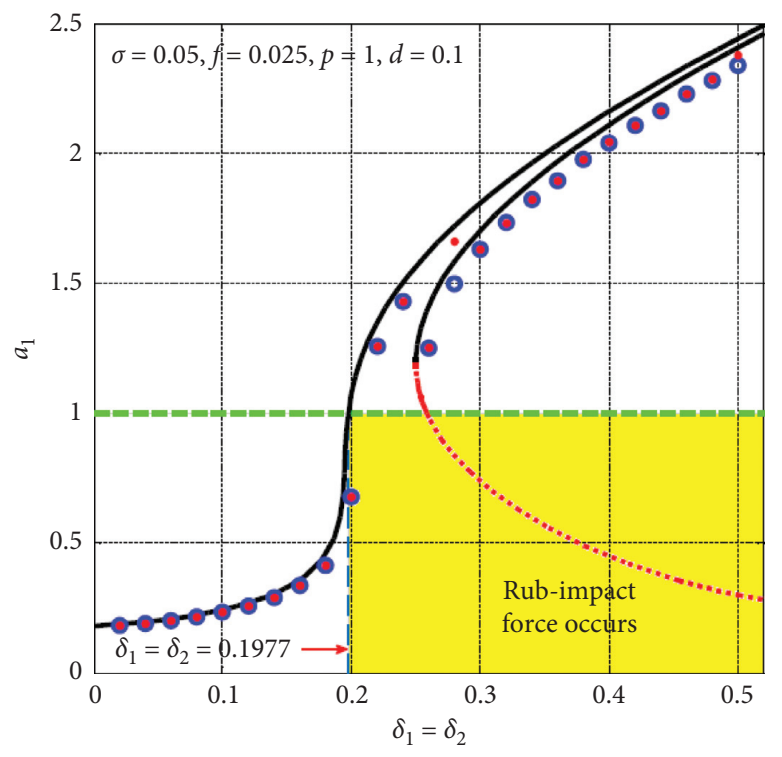

(c)

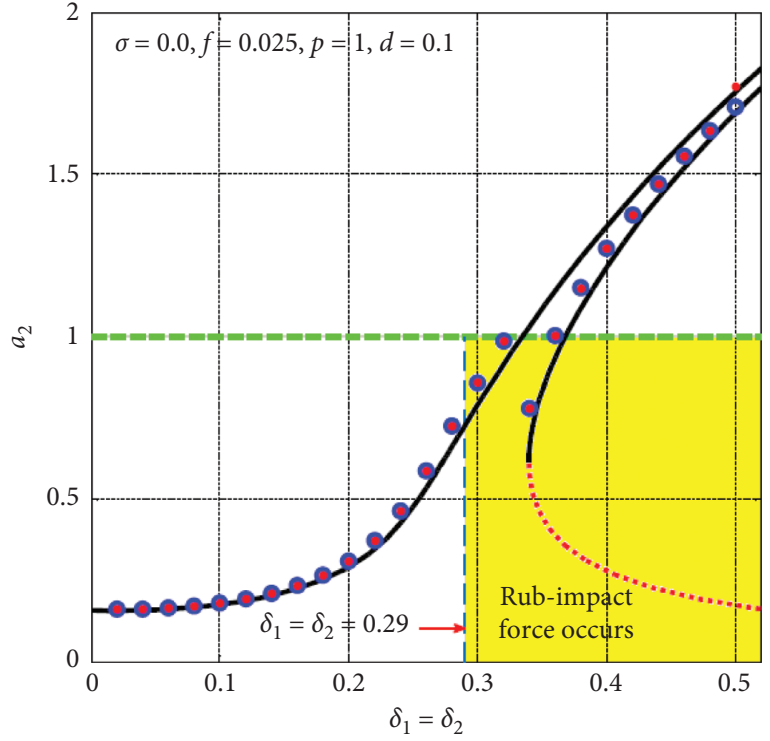

(b)

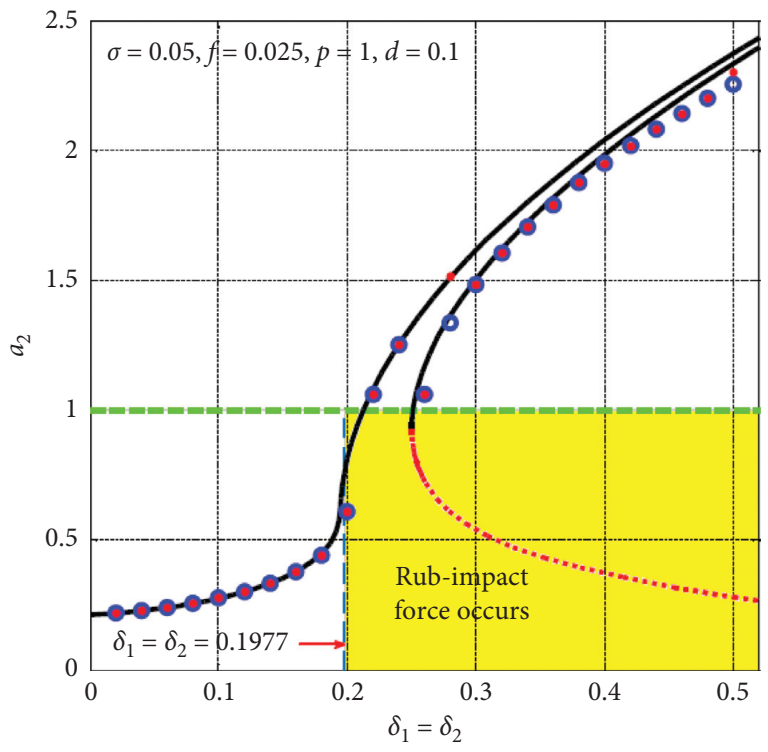

(d)

FIGURE 18: Controlled system $\delta_{1}=\delta_{2}$ response curves when the proportional control gain $p=1.0$ and derivative control gain $d=0.1$ at two different values of the detuning parameter $\sigma:(\mathrm{a}, \mathrm{b}) \delta_{1}=\delta_{2}$ response curves when $\sigma=0.0$ and (c, d) $\delta_{1}=\delta_{2}$ response curves when $\sigma=0.05$. 


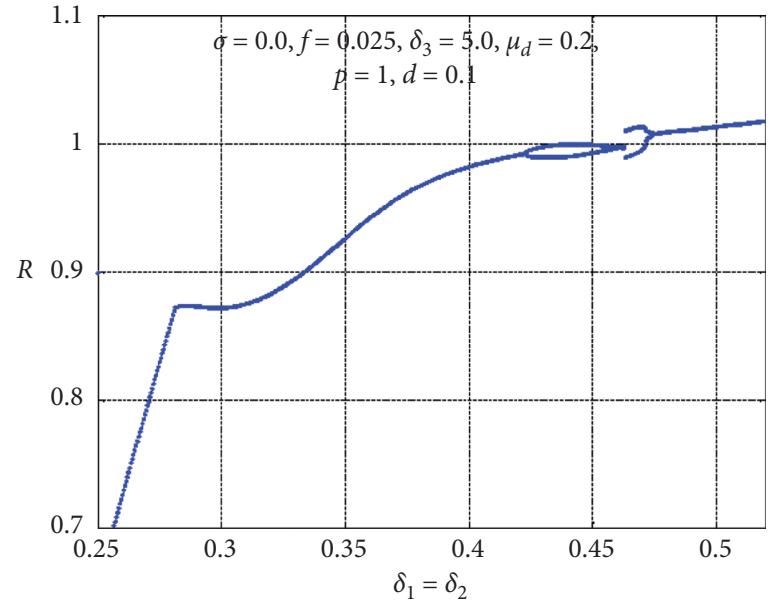

(a)

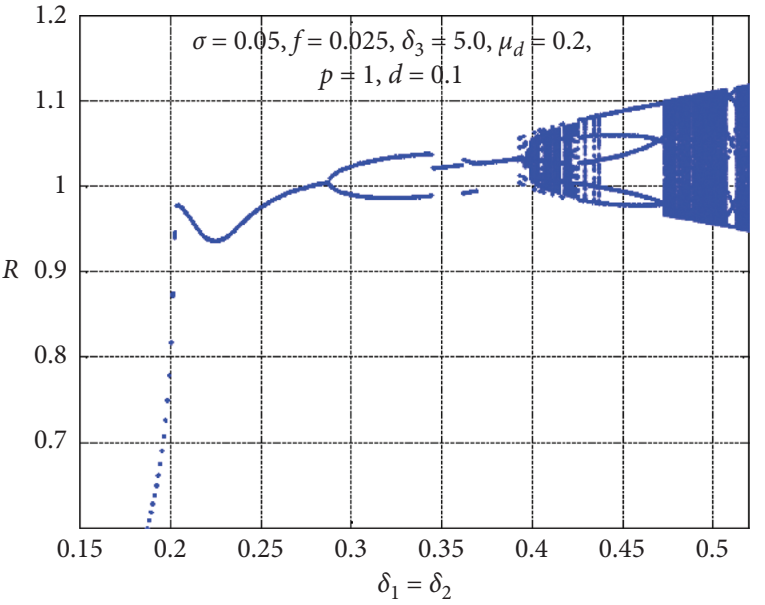

(b)

Figure 19: Bifurcation diagram of the controlled system according to Figure 18 (i.e., $p=1.0, d=0.1$ ): (a) bifurcation diagram according to Figures 18(a) and 18(b) when $\sigma=0.0$; (b) bifurcation diagram according to Figures 18(c) and 18(d) (i.e., when $\sigma=0.05$ ).

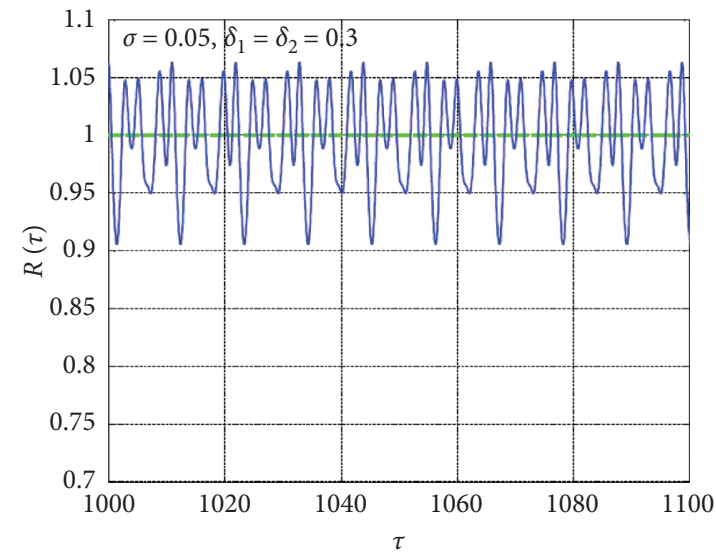

(a)

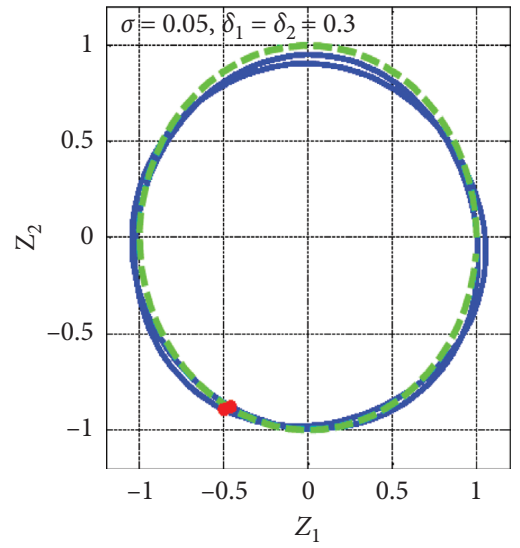

(b)

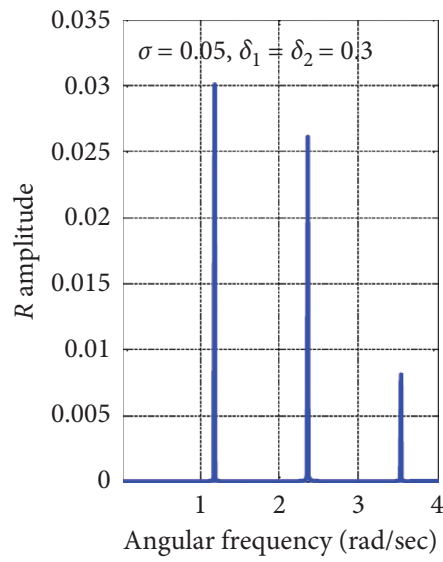

(c)

Figure 20: Controlled system time response (i.e., $p=1.0, d=0.1$ ) according to Figure 19(b) when $\delta_{1}=\delta_{2}=0.3$ : (a) the temporal oscillation of the radial displacement $R(\tau)=\sqrt{z_{1}^{2}+z_{2}^{2}}$, (b) whirling orbit $z_{1}(\tau)$ versus $z_{2}(\tau)$, and (c) frequency spectrum.

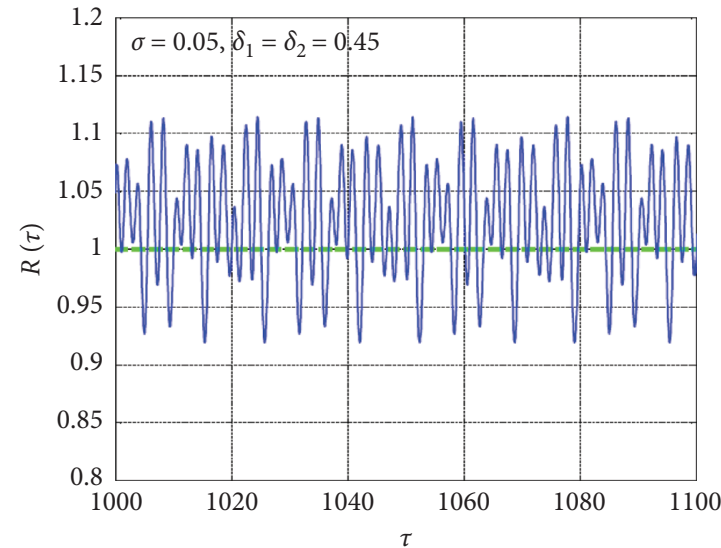

(a)

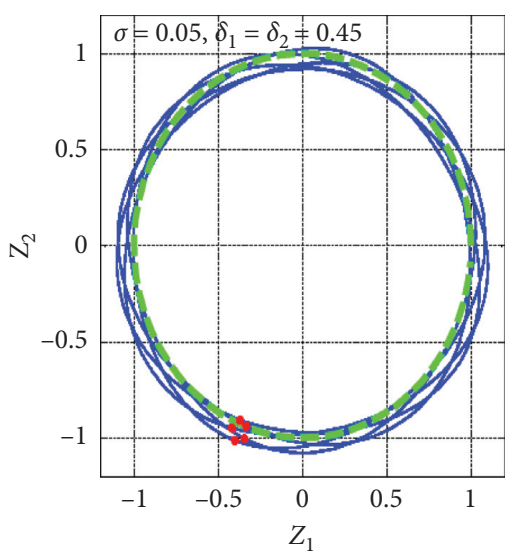

(b)

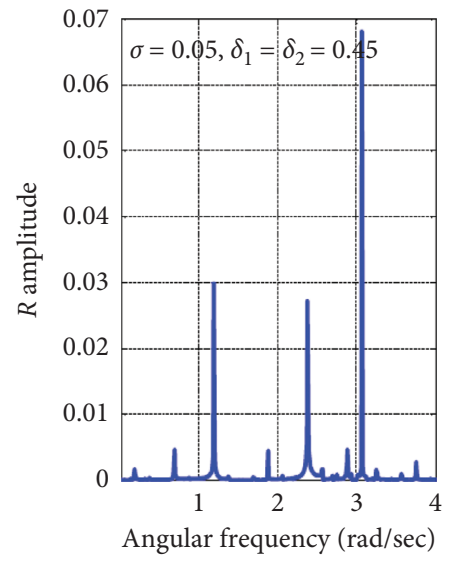

(c)

FIgURe 21: Controlled system time response (i.e., $p=1.0, d=0.1$ ) according to Figure 19(b) when $\delta_{1}=\delta_{2}=0.45$ : (a) the temporal oscillation of the radial displacement $R(\tau)=\sqrt{z_{1}^{2}+z_{2}^{2}}$, (b) whirling orbit $z_{1}(\tau)$ versus $z_{2}(\tau)$, and (c) frequency spectrum. 


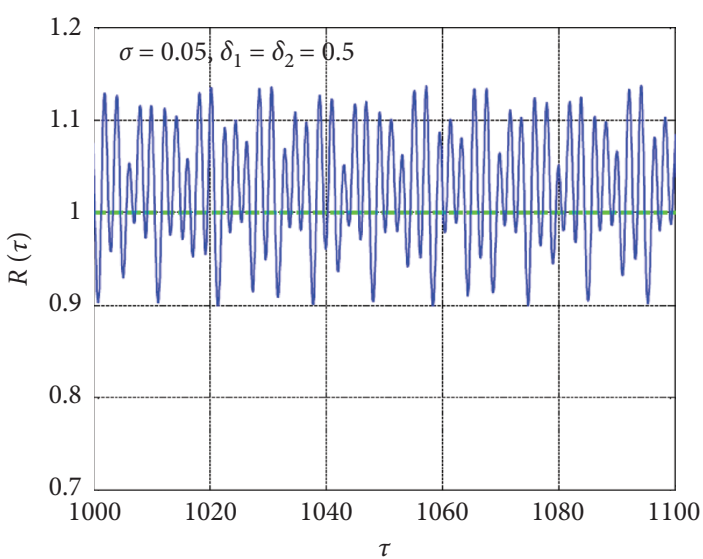

(a)

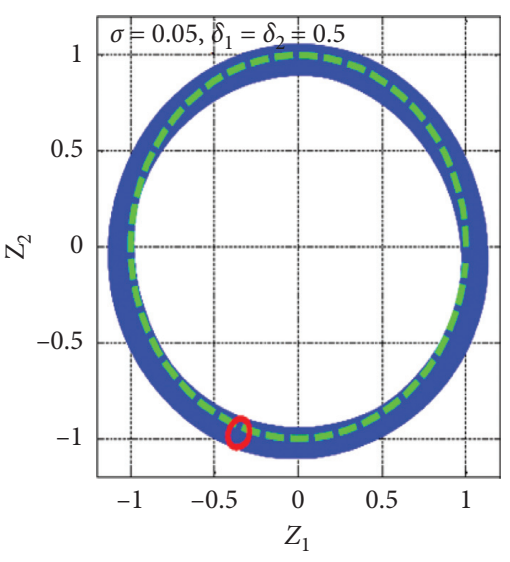

(b)

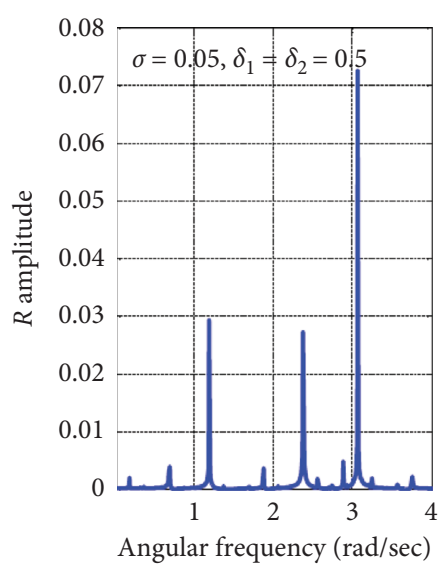

(c)

Figure 22: Controlled system time response (i.e., $p=1.0, d=0.1$ ) according to Figure 19(b) when $\delta_{1}=\delta_{2}=0.5$ : (a) the temporal oscillation of the radial displacement $R(\tau)=\sqrt{z_{1}^{2}+z_{2}^{2}}$, (b) whirling orbit $z_{1}(\tau)$ versus $z_{2}(\tau)$, and (c) frequency spectrum.

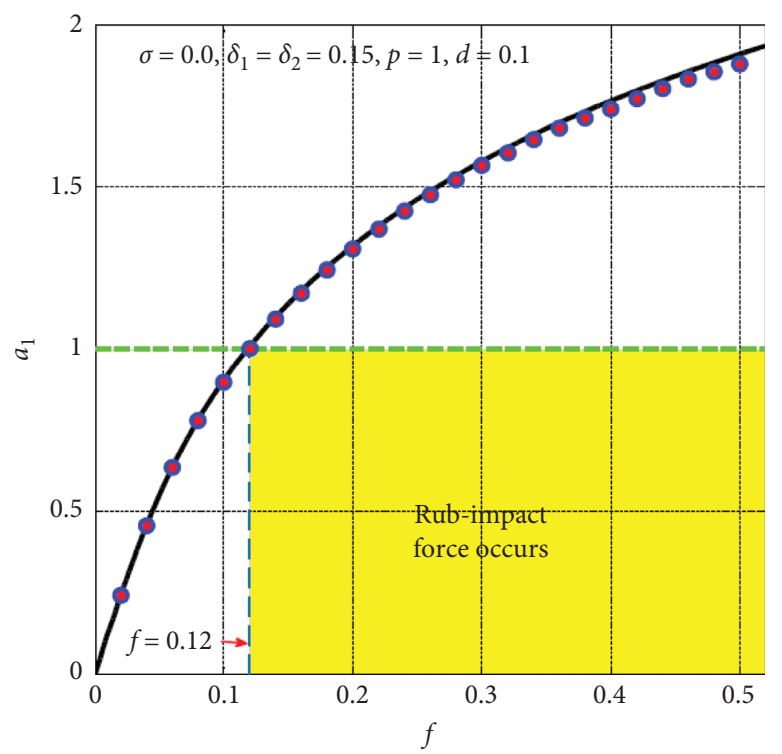

(a)

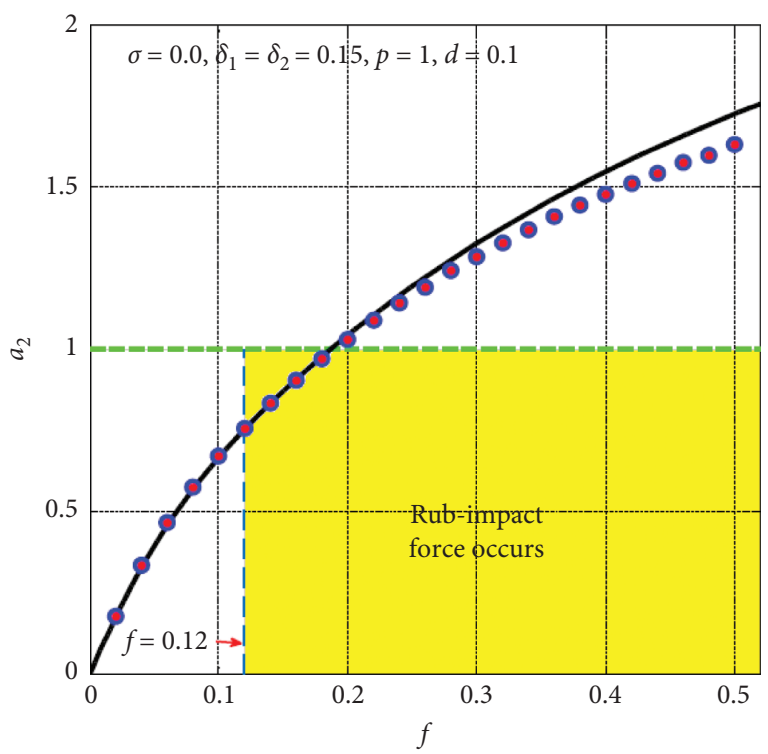

(b)

FIgURE 23: Continued. 


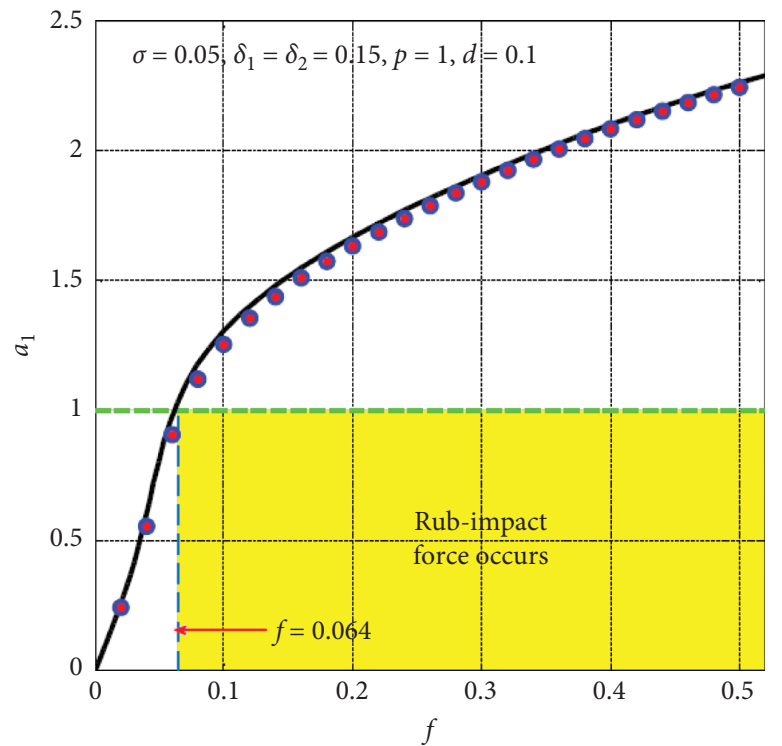

(c)

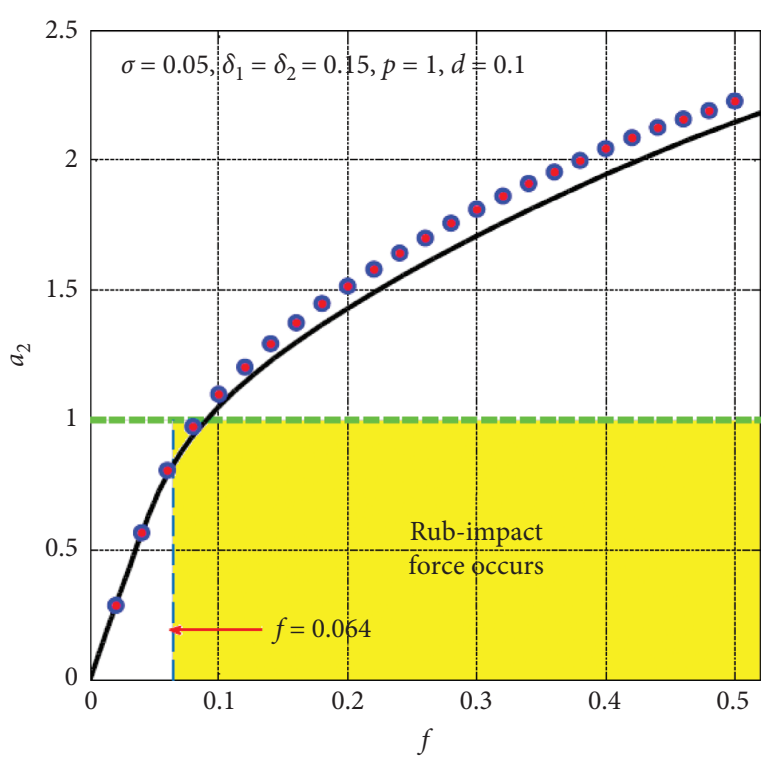

(d)

FIGURE 23: Controlled system eccentricity response curves when the proportional control gain $p=1.0$, derivative control gain $d=0.1$, and $\delta_{1}=\delta_{2}=0.15$ at two different values of the detuning parameter $\sigma$ : (a,b) eccentricity response curves when $\sigma=0.0$ and (c, d) eccentricity response curves when $\sigma=0.05$.

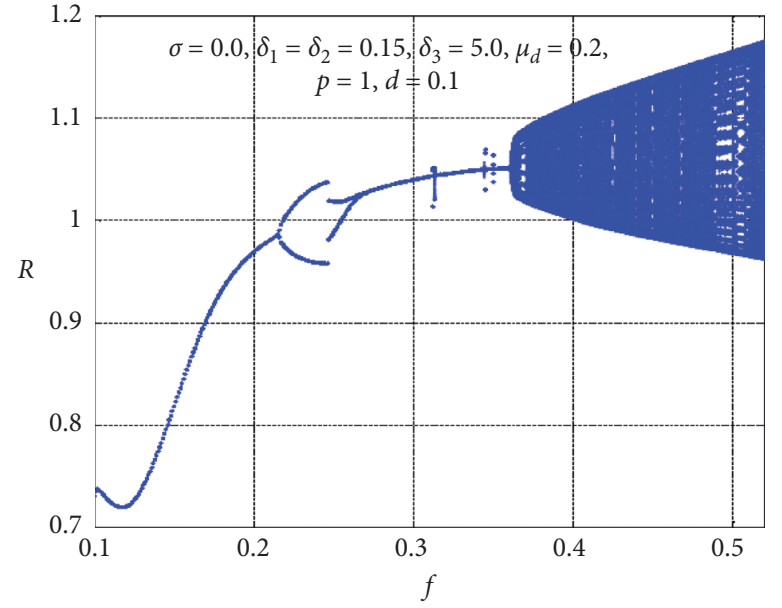

(a)

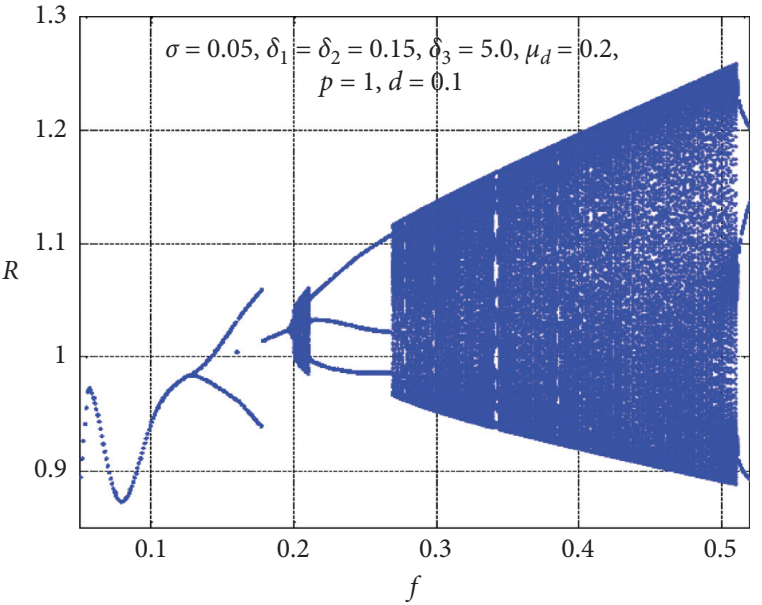

(b)

Figure 24: Bifurcation diagram of the controlled system according to Figure 23 (i.e., $p=1.0, d=0.1, \delta_{1}=\delta_{2}=0.15$ ): (a) bifurcation diagram according to Figures 23(a) and 23(b) when $\sigma=0.0$; (b) bifurcation diagram according to Figures 23(c) and 23(d) (i.e., when $\sigma=0.05)$.

Figure 24(b)), but the system can exhibit periodic, period-n, quasiperiodic, or chaotic motion when the rub and impact forces occur.

\section{Conclusions}

The nonlinear oscillation control of an asymmetric horizontally suspended rotor is discussed in this article. The rotor is modeled as a two-degree-of-freedom discontinuous dynamical system with asymmetric stiffness. A proportional-derivative control law is proposed to mitigate the lateral oscillations of the considered rotor system. It is suggested to couple the proposed controller to the rotor system via poles' active magnetic bearings fixed on the stator housing. The whole system model (i.e., the rotor system and the proposed controller) is derived considering the rub and impact forces between the rotor and stator housing. The obtained system model is investigated in two steps. Firstly, the rub and impact forces are neglected to analyze the periodic response and determine the conditions at which the system can exhibit rub and impact between the rotor and stator applying the 
asymptotic analysis. Secondly, the nature of the system motions is explored via plotting the different bifurcation diagrams, temporal oscillations, whirling orbits, and frequency spectrum of the system model including the rub and impact forces. According to the introduced analysis and discussion, some conclusions can be summarized as follows:

(a) The rotor oscillation amplitudes are monotonic increasing functions of both the disk eccentricity and the asymmetric stiffness coefficients.

(b) The uncontrolled system exhibits many nonlinear phenomena such as the sensitivity to the initial conditions, jump phenomenon, and the coexistence of multiple whirling orbits.

(c) The rotating shaft system exhibits periodic motions only as long as the rub and impact forces do not occur between the rotor and the stator housing.

(d) The occurrence of rub and impact forces results in the oscillation of the system with period-n, quasiperiodic, or chaotic motion depending on the disk spinning speed.

(e) It is possible to force the nonlinear rotor system to respond as a linear system without rub and impact forces via the proper design of the control parameters $(p$ and $d)$.

\section{Nomenclature}

\begin{tabular}{|c|c|}
\hline$z_{1}, \dot{z}_{1}, \ddot{z}_{1}:$ & $\begin{array}{l}\text { The horizontal displacement, } \\
\text { horizontal velocity, and horizontal } \\
\text { acceleration of the rotor system }\end{array}$ \\
\hline$z_{2}, \dot{z}_{2}, \ddot{z}_{2}$ & $\begin{array}{l}\text { The vertical displacement, vertical } \\
\text { velocity, and vertical acceleration of the } \\
\text { rotor system }\end{array}$ \\
\hline$R:$ & $\begin{array}{l}\text { The rotor system instantaneous radial } \\
\text { displacement, where } R=\sqrt{z_{1}^{2}+z_{2}^{2}}\end{array}$ \\
\hline$\mu_{1}, \mu_{2}:$ & $\begin{array}{l}\text { Horizontal and vertical linear damping } \\
\text { coefficients, respectively }\end{array}$ \\
\hline$\omega_{1}, \omega_{2}:$ & $\begin{array}{l}\text { Horizontal and vertical natural } \\
\text { frequencies, respectively }\end{array}$ \\
\hline$\eta, \beta:$ & $\begin{array}{l}\text { Quadratic and cubic nonlinearity } \\
\text { coefficients, respectively }\end{array}$ \\
\hline$\delta_{1}, \delta_{2}:$ & $\begin{array}{l}\text { Linear symmetric stiffness coefficient } \\
\text { and nonlinear symmetric stiffness } \\
\text { coefficient, respectively }\end{array}$ \\
\hline$\delta_{3}:$ & $\begin{array}{l}\text { Stiffness coefficient of impact between } \\
\text { the rotating shaft and pole legs }\end{array}$ \\
\hline$\mu_{d}:$ & Dynamic friction coefficient \\
\hline$f:$ & The eccentricity of the rotating disk \\
\hline$\Omega:$ & Shaft spinning speed \\
\hline$p, d:$ & $\begin{array}{l}\text { Proportional and derivative control } \\
\text { gains, respectively }\end{array}$ \\
\hline$\alpha_{i},(i=1,2, \ldots, 5):$ & Control parameters function of $p$ and $d$ \\
\hline$a_{1}, a_{2}:$ & $\begin{array}{l}\text { Vibration amplitudes in } z_{1} \text { and } z_{2} \\
\text { directions, respectively, when the rub- } \\
\text { impact force is neglected }\end{array}$ \\
\hline$H:$ & Heaviside function. \\
\hline
\end{tabular}

\section{Data Availability}

The data used to support the findings of this study are included within the article.

\section{Conflicts of Interest}

The authors declare that there are no potential conflicts of interest concerning the research, authorship, and/or publication of this article.

\section{Acknowledgments}

The authors extend their appreciation to King Saud University for funding this work through Researchers Supporting Project (no. RSP-2021/252), King Saud University, Riyadh, Saudi Arabia. This study was also supported by the National Science Centre, Poland, under the grant OPUS 14 no. 2017/27/B/ST8/01330. This study was also supported by the National Science Centre, Poland, under the grant OPUS 14 no. 2017/27/B/ST8/01330.

\section{References}

[1] T. Iwatsubo, Y. Tsujiuchi, and T. Inoue, "Vibration of asymmetric rotor supported by oil film bearings," IngenieurArchiv, vol. 56, no. 1, pp. 1-15, 1986.

[2] J. P. Park, "Diagnosis of excessive vibration signals of twoPole generator rotors in balancing," KSME International Journal, vol. 14, no. 6, pp. 590-596, 2000.

[3] S. Hsieh, J. Chen, and A. Lee, "A modified transfer matrix method for the coupled lateral and torsional vibrations of asymmetric rotor-bearing systems," Journal of Sound and Vibration, vol. 312, no. 4-5, pp. 563-571, 2008.

[4] Y. Ishida and J. Liu, "Elimination of unstable ranges of rotors utilizing discontinuous spring characteristics: an asymmetrical shaft system, an asymmetrical rotor system, and a rotor system with liquid," Journal of Vibration and Acoustics, vol. 132, no. 1, Article ID 011011, 2010.

[5] M. Shahgholi and S. E. Khadem, "Primary and parametric resonances of asymmetrical rotating shafts with stretching nonlinearity," Mechanism and Machine Theory, vol. 51, pp. 131-144, 2012.

[6] Q. Han and F. Chu, "The effect of transverse crack upon parametric instability of a rotor-bearing system with an asymmetric disk," Communications in Nonlinear Science and Numerical Simulation, vol. 17, no. 12, pp. 5189-5200, 2012.

[7] Q. Han and F. Chu, "Parametric instability of a Jeffcott rotor with rotationally asymmetric inertia and transverse crack," Nonlinear Dynamics, vol. 73, no. 1-2, pp. 827-842, 2013.

[8] N. A. Saeed and M. Eissa, "Bifurcation analysis of a transversely cracked nonlinear Jeffcott rotor system at different resonance cases," International Journal of Acoustics and Vibration, vol. 24, no. 2, pp. 284-302, 2019.

[9] N. A. Saeed and M. Eissa, "Bifurcations of periodic motion of a horizontally supported nonlinear Jeffcott rotor system having transversely cracked shaft," International Journal of Non-linear Mechanics, vol. 101, pp. 113-130, 2018.

[10] N. A. Saeed, M. S. Mohamed, and S. K. Elagan, "Periodic, quasi-periodic, and chaotic motions to diagnose a crack on a horizontally supported nonlinear rotor system," Symmetry, vol. 12, p. 2059, 2020. 
[11] L. Xiang, X. Gao, and A. Hu, "Nonlinear dynamics of an asymmetric rotor-bearing system with coupling faults of crack and rub-impact under oil-film forces," Nonlinear Dynamics, vol. 86, no. 2, pp. 1057-1067, 2016.

[12] P. M. Przybylowicz, Z. Starczewski, and K. P. Korczak, "Sensitivity of regions of irregular and chaotic vibrations of an asymmetric rotor supported on journal bearings to structural parameters," Acta Mechanica, vol. 227, no. 11, pp. 3101-3112, 2016.

[13] N. A. Saeed, "On vibration behavior and motion bifurcation of a nonlinear asymmetric rotating shaft," Archive of Applied Mechanics, vol. 89, no. 9, pp. 1899-1921, 2019.

[14] N. A. Saeed, "On the steady-state forward and backward whirling motion of asymmetric nonlinear rotor system," European Journal of Mechanics-A: Solids, vol. 80, Article ID 103878,2020

[15] J. L. P. Felix, J. M. Balthazar, and R. M. L. R. F. Brasilv, "A short note on transverse vibrations of a shaft carrying two (or one) disk excited by a nonideal motor," Journal of Computational and Nonlinear Dynamics, vol. 4, Article ID 014501, 2009.

[16] G. G. Tehrani and M. Dardel, "Mitigation of nonlinear oscillations of a Jeffcott rotor system with an optimized damper and nonlinear energy sink," International Journal of Nonlinear Mechanics, vol. 98, pp. 122-136, 2018.

[17] G. G. Tehrani, M. Dardel, and M. H. Pashaei, "Passive vibration absorbers for vibration reduction in the multi-bladed rotor with rotor and stator contact," Acta Mechanica, vol. 231, no. 2, pp. 597-623, 2020.

[18] Y. Ishida and T. Inoue, "Vibration suppression of nonlinear rotor systems using a dynamic damper," Journal of Vibration and Control, vol. 13, no. 8, pp. 1127-1143, 2007.

[19] N. A. Saeed and M. Kamel, "Nonlinear PD-controller to suppress the nonlinear oscillations of horizontally supported Jeffcott-rotor system," International Journal of Non-Linear Mechanics, vol. 87, pp. 109-124, 2016.

[20] N. A. Saeed, E. M. Awwad, M. A. E. Meligy, and E. A. Nasr, "Sensitivity analysis and vibration control of asymmetric nonlinear rotating shaft system utilizing 4-pole AMBs as an actuator," European Journal of Mechanics-A: Solids, vol. 86, Article ID 104145, 2021.

[21] N. A. Saeed, E. Mahrous, and J. Awrejcewicz, "Nonlinear dynamics of the six-pole rotor-AMB system under two different control configurations," Nonlinear Dynamics, vol. 101, no. 4, pp. 2299-2323, 2020.

[22] N. A. Saeed, E. M. Awwad, M. A. E. Meligy, and E. S. A. Nasr, "Radial versus Cartesian control strategies to stabilize the nonlinear whirling motion of the six-pole rotor-AMBs," IEEE Access, vol. 8, Article ID 138859, 2020.

[23] V. Piccirillo, J. M. Balthazar, and A. M. Tusset, "Chaos control and impact suppression in rotor-bearing system using magnetorheological fluid," The European Physical Journal-Special Topics, vol. 224, no. 14-15, pp. 3023-3040, 2015.

[24] J. P. Singh, B. K. Roy, and N. V. Kuznetsov, "Multistability and hidden attractors in the dynamics of permanent magnet synchronous motor," International Journal of Bifurcation and Chaos, vol. 29, no. 4, Article ID 1950056, 2019.

[25] N. A. Saeed, E. M. Awwad, M. A. E. Meligy, and E. A. Nasr, "Analysis of the rub-impact forces between a controlled nonlinear rotating shaft system and the electromagnet pole legs," Applied Mathematical Modelling, vol. 93, pp. 792-810, 2021.

[26] H. Yabuno, T. Kashimura, T. Inoue, and Y. Ishida, "Nonlinear normal modes and primary resonance of horizontally supported Jeffcott rotor," Nonlinear Dynamics, vol. 66, no. 3, pp. 377-387, 2011.

[27] N. A. Saeed and H. A. E. Gohary, "On the nonlinear oscillations of a horizontally supported Jeffcott rotor with a nonlinear restoring force," Nonlinear Dynamics, vol. 88, no. 1, pp. 293-314, 2017.

[28] N. A. Saeed, S. I. E. Bendary, M. Sayed, M. S. Mohamed, and S. K. Elagan, "On the oscillatory behaviours and rub-impact forces of a horizontally supported asymmetric rotor system under position-velocity feedback controller," Latin American Journal of Solids and Structures, vol. 18, no. 2, 2021.

[29] Y. Ishida and T. Yamamoto, Linear and Nonlinear Rotordynamics, Wiley VCH, Hoboken, NJ, USA, 2012.

[30] H. Bleuler, M. Cole, and P. Keogh, Magnetic Bearings Theory, Design, and Application to Rotating Machinery, Springer, Berlin, Germany, 2009.

[31] A. Nayfeh and D. Mook, Nonlinear Oscillations, Wiley, Hoboken, NY, USA, 1995.

[32] A. H. Nayfeh, "Resolving controversies in the application of the method of multiple scales and the generalized method of averaging," Nonlinear Dynamics, vol. 40, no. 1, pp. 61-102, 2005. 